\title{
Infinitene: A Helically Twisted Figure-Eight [12]Circulene Topoisomer
}

\author{
Maciej Krzeszewski, ${ }^{\dagger}$ Hideto Ito, ${ }^{*}+{ }^{\dagger}$ and Kenichiro Itami ${ }^{*},+$, \\ ${ }^{\dagger}$ Department of Chemistry, Graduate School of Science, Nagoya University, Nagoya 464-8602, \\ Japan. \\ ‡ Institute of Transformative Bio-Molecules (WPI-ITbM), Nagoya University, Nagoya 464-8602, \\ Japan \\ *itami@chem.nagoya-u.ac.jp (K.I.) \\ * ito.hideto@g.mbox.nagoya-u.ac.jp (H.I.)
}

\section{Table of contents}

1. General remarks S2

2. Experimental section S3

3. X-ray crystallographic analysis $\quad \mathrm{S10}$

4. Photophysical properties $\quad$ S16

5. Chiral HPLC analyses and CD spectra $\quad \mathrm{S} 17$

6. Circularly polarized luminescence S18

7. DFT calculations $\quad \mathrm{S19}$

8. Cartesian coordinates of optimized structures S23

9. References $\quad$ S28

10. ${ }^{1} \mathrm{H}$ and ${ }^{13} \mathrm{C}$ NMR spectra of synthesized compounds $\quad \mathrm{S} 29$ 


\section{General remarks}

Unless otherwise noted, all materials including dry solvents were obtained from commercial suppliers and used without further purification. All reagents were purchased from $\mathrm{TCl}$, Aldrich or Wako. Starting materials $\mathbf{S} \mathbf{1}^{[1]}$ and $\mathbf{S} \mathbf{4}^{[2]}$ were synthesized according to the procedures reported in the literature. Unless otherwise noted, all reactions were performed with dry solvents under an atmosphere of nitrogen in oven-dried glassware with standard vacuum-line techniques. All work-up and purification procedures were carried out with reagent-grade solvents in air. Analytical thin-layer chromatography (TLC) was performed using E. Merck silica gel $60 \mathrm{~F}_{254}$ precoated plates $(0.25 \mathrm{~mm})$. The developed chromatograms were analyzed by UV lamp $(254 \mathrm{~nm})$. Flash column chromatography was performed with KANTO Silica Gel 60N (spherical, neutral, 40-100 $\mu \mathrm{m}$ ) or Biotage Isolera ${ }^{\circledR}$ equipped with Biotage SNAP Cartridge KP-Sil columns. Preparative thin-layer chromatography (PTLC) was performed using Wako-gel ${ }^{\circledR}$ B5-F silica coated plates $(0.75 \mathrm{~mm})$ prepared in our laboratory. Gas chromatography (GC) analysis was conducted on a Shimadzu GC-2010 instrument equipped with a HP-5 column (30 m × $0.25 \mathrm{~mm}$, Hewlett-Packard). High-resolution mass spectra (HRMS) were obtained from a Thermo Fisher Scientific Exactive (APCI or ESI) and a JEOL JMST100GCV (Direct EI). Nuclear magnetic resonance (NMR) spectra were recorded on a JEOL ECS$600\left({ }^{1} \mathrm{H} 600 \mathrm{MHz},{ }^{13} \mathrm{C} 151 \mathrm{MHz}\right)$ spectrometer and a JEOL ECA 600II with Ultra COOL ${ }^{\mathrm{TM}}$ probe $\left({ }^{1} \mathrm{H}\right.$ $600 \mathrm{MHz},{ }^{13} \mathrm{C} 151 \mathrm{MHz}$ ). Chemical shifts for ${ }^{1} \mathrm{H}$ NMR are expressed in parts per million (ppm) relative to tetramethylsilane $(\delta 0.00 \mathrm{ppm}), \mathrm{CDCl}_{3}(\delta \quad 7.26 \mathrm{ppm}), \mathrm{CD}_{2} \mathrm{Cl}_{2}(\delta 5.32 \mathrm{ppm}), 1,1,2,2-$ tetrachloroethane (TCE- $d_{2}, \delta 6.00 \mathrm{ppm}$ ), dimethylsulfoxide (DMSO- $\left.d_{6}, \delta 2.50 \mathrm{ppm}\right)$ or acetone- $d_{6}(\delta$ $2.05 \mathrm{ppm})$. Chemical shifts for ${ }^{13} \mathrm{C}$ NMR are expressed in ppm relative to $\mathrm{CDCl}_{3}(\delta 77.0 \mathrm{ppm}), \mathrm{CD}_{2} \mathrm{Cl}_{2}$ ( $\delta 53.8 \mathrm{ppm}$ ), TCE- $d_{2}\left(\delta 73.8 \mathrm{ppm}\right.$ ), dimethylsulfoxide (DMSO- $d_{6}, \delta 39.5 \mathrm{ppm}$ ) or acetone- $d_{6}(\delta 29.8$ ppm). Data are reported as follows: chemical shift, multiplicity ( $s=$ singlet, $d=$ doublet, $d d=$ doublet of doublets, $\mathrm{ddd}=$ doublet of doublet of doublets, $\mathrm{t}=$ triplet, $\mathrm{t} d=$ triplet of doublets, $\mathrm{q}=$ quartet, $\mathrm{m}=$ multiplet), coupling constant $(\mathrm{Hz})$, and integration. Chiral HPLC analysis was conducted on a Shimadzu Prominence 2000 instrument equipped with CHIRALPAK ${ }^{\circledR}$ IE column (10 mm $\times 250 \mathrm{~mm}$ ). UV-vis absorption spectra were measured with a Shimadzu UV-3150 spectrometer. Circular dichroism spectra were measured with a JASCO FT/IR6100. Steady-state fluorescence measurements were performed with dilute solutions $\left(10^{-6} \mathrm{M}\right.$, optical density $\left.<0.1\right)$ contained in standard $1 \mathrm{~cm}$ quartz cuvettes at room temperature. Emission spectra were recorded on an F-4500 Hitachi spectrometer with a resolution of $0.4 \mathrm{~nm}$ and automatically corrected by instrumental function. Absolute fluorescence quantum yields were determined with a Hamamatsu C9920-02 calibrated integrating sphere system equipped with multichannel spectrometer (PMA-11). Circularly polarized luminescence $(\mathrm{CPL})$ spectra were recorded on a JASCO CPL-300 spectrofluoropolarimeter at $25^{\circ} \mathrm{C}$ with $10 \times 10 \mathrm{~mm}$ quartz cells under the following conditions: scattering angle: $0^{\circ}$, excitation slit width: $5 \mathrm{~nm}$, emission slit width: $5 \mathrm{~nm}$, scan rate: $100 \mathrm{~nm} \mathrm{~min}^{-1}$, response: $4 \mathrm{~s}$, accumulation: 8 times; data interval: $0.5 \mathrm{~nm}$, solvent: $\mathrm{CH}_{2} \mathrm{Cl}_{2}$, excitation wavelength: $400 \mathrm{~nm}, \mathrm{HT}$ voltage (photomultiplier): 650 $\mathrm{V}$. 


\section{Experimental section}

\subsection{Preparation of precursors 2 and 3.}

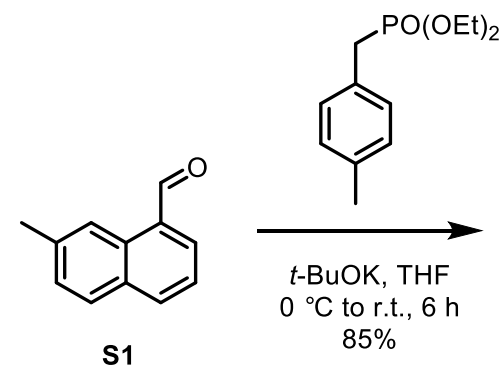

s1<smiles>Cc1ccc(/C=C/c2cccc3ccc(C)cc23)cc1</smiles>

S2

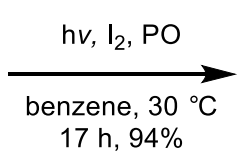
benzoyl peroxide.

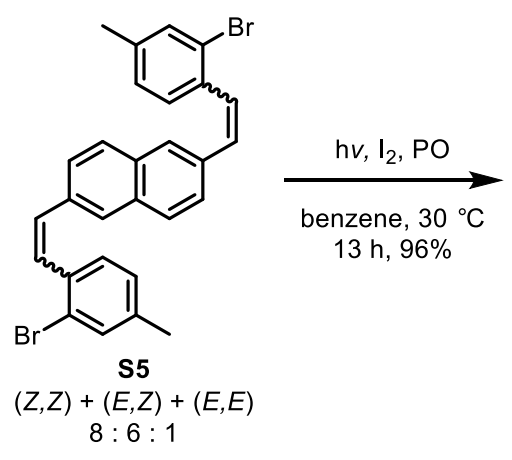

S6

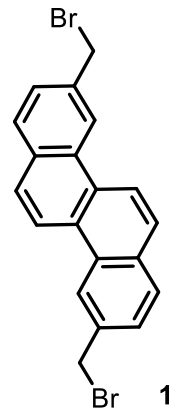

Scheme S1. Synthetic approach towards 3,9-bis(bromomethyl)chrysene (2). PO: propylene oxide, BPO:

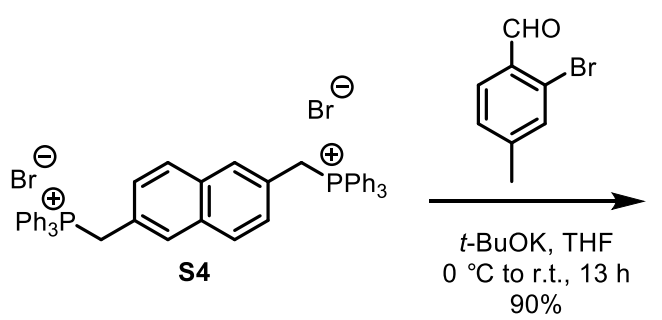

$90 \%$

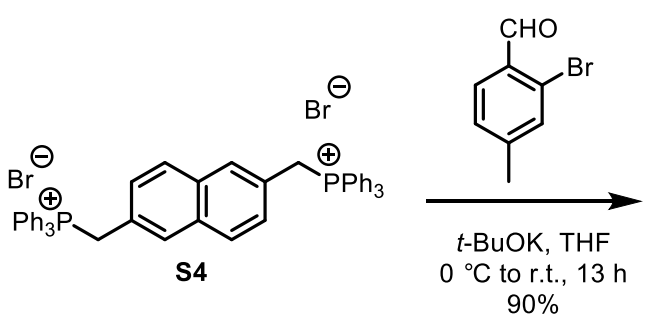

1. $n$-BuLi, THF $-78{ }^{\circ} \mathrm{C}$ to $0^{\circ} \mathrm{C}, 30 \mathrm{~min}$

2. $\mathrm{H}_{2} \mathrm{O}, 0{ }^{\circ} \mathrm{C}$ to r.t. $30 \mathrm{~min}, 95 \%$

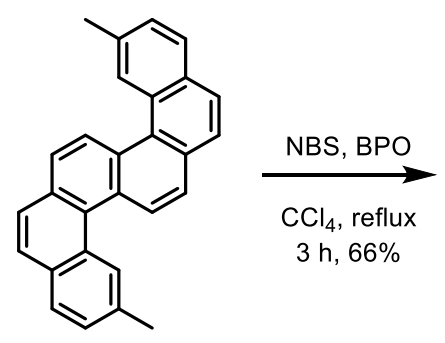

S7

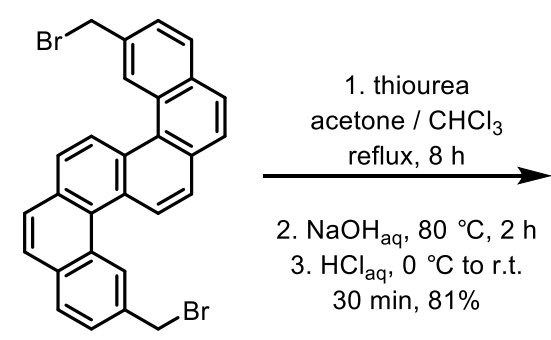

S8<smiles></smiles>

2

Scheme S2. Synthetic approach towards dibenzo[c, ]chrysene-3,11-diyldimethanethiol (3). PO: propylene oxide, BPO: benzoyl peroxide.

\section{7-Methyl-1-naphthaldehyde (S1)}

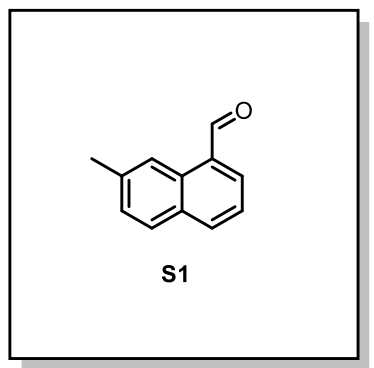

S1 was synthesized according to the literature procedure. ${ }^{1}{ }^{1} \mathrm{H}$ NMR $(600$ $\left.\mathrm{MHz} \mathrm{CDCl}_{3}\right) \delta 10.38(\mathrm{~s}, 1 \mathrm{H}), 9.06(\mathrm{~d}, J=0.7 \mathrm{~Hz}, 1 \mathrm{H}), 8.05(\mathrm{~d}, J=8.1 \mathrm{~Hz}, 1 \mathrm{H})$, $7.95(\mathrm{dd}, J=7.0,1.1 \mathrm{~Hz}, 1 \mathrm{H}), 7.81(\mathrm{~d}, J=8.3 \mathrm{~Hz}, 1 \mathrm{H}), 7.55(\mathrm{dd}, J=7.2,7.2$ $\mathrm{Hz}, 1 \mathrm{H}), 7.43(\mathrm{dd}, J=8.3,1.5 \mathrm{~Hz}, 1 \mathrm{H}), 2.59(\mathrm{~s}, 3 \mathrm{H}) .{ }^{13} \mathrm{C}$ NMR $(151 \mathrm{MHz}$, $\left.\mathrm{CDCl}_{3}\right) \delta 193.7,139.4,136.9,135.1,132.0,130.9,130.8,129.1,128.2$, $123.9(2 \mathrm{C}), 22.3$. Spectral properties were in agreement with those previously reported. 


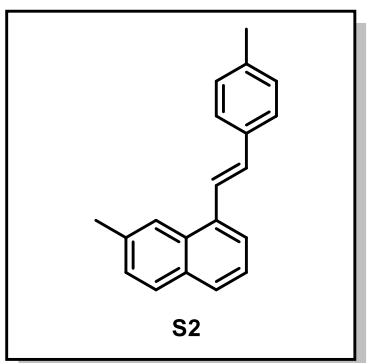

from ethanol to afford $\mathbf{S 2}(2.45 \mathrm{~g}, 85 \%)$ as pale-yellow needles. ${ }^{1} \mathrm{H}$ NMR $\left(600 \mathrm{MHz}, \mathrm{CDCl}_{3}\right) \delta 7.96$ $(\mathrm{s}, 1 \mathrm{H}), 7.81(\mathrm{~d}, J=16.2 \mathrm{~Hz}, 1 \mathrm{H}), 7.76(\mathrm{~d}, J=8.3 \mathrm{~Hz}, 1 \mathrm{H}), 7.74(\mathrm{~d}, J=8.3 \mathrm{~Hz}, 1 \mathrm{H}), 7.69(\mathrm{~d}, J=7.1$ $\mathrm{Hz}, 1 \mathrm{H}), 7.51(\mathrm{~d}, J=8.3 \mathrm{~Hz}, 2 \mathrm{H}), 7.40(\mathrm{t}, J=7.2 \mathrm{~Hz}, 1 \mathrm{H}), 7.33(\mathrm{dd}, J=8.4,1.5 \mathrm{~Hz}, 1 \mathrm{H}), 7.21(\mathrm{~d}, J=$ $7.9 \mathrm{~Hz}, 2 \mathrm{H}), 7.11(\mathrm{~d}, J=16.2 \mathrm{~Hz}, 1 \mathrm{H}), 2.55(\mathrm{~s}, 3 \mathrm{H}), 2.39(\mathrm{~s}, 3 \mathrm{H}) \cdot{ }^{13} \mathrm{C} \mathrm{NMR}\left(151 \mathrm{MHz}, \mathrm{CDCl}_{3}\right) \delta 137.6$, $135.7,135.0,134.5,131.9,131.6,131.4,129.4,128.4,128.0,127.6,126.6,125.1,124.8,123.6$, 122.9, 22.1, 21.3. HRMS $\left(\mathrm{APCl}^{+}\right) \mathrm{m} / \mathrm{z}$ calcd for $\mathrm{C}_{20} \mathrm{H}_{19}[\mathrm{M}+\mathrm{H}]^{+}:$259.1481, found 259.1482.

\section{3,9-Dimethylchrysene (S3)}

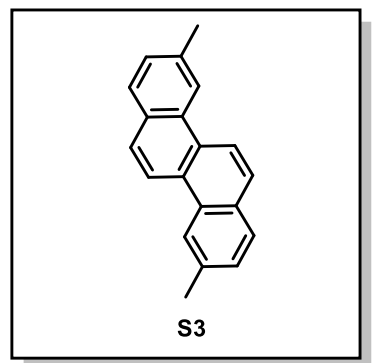

Argon was bubbled through a stirred solution of $\mathbf{S 2}(439 \mathrm{mg}, 1.70 \mathrm{mmol})$ and iodine $(1.30 \mathrm{~g}, 5.10 \mathrm{mmol})$ in dry benzene $(500 \mathrm{~mL})$ for $30 \mathrm{~min}$. Then, $3.0 \mathrm{~mL}$ of propylene oxide was added, and the reaction mixture was irradiated with a $450 \mathrm{~W}$ UV lamp and stirred for additional $24 \mathrm{~h}$ at $30^{\circ} \mathrm{C}$. After the reaction was completed, the mixture was washed with aqueous $\mathrm{Na}_{2} \mathrm{~S}_{2} \mathrm{O}_{3}$ solution, water, and brine. The organic layer was dried with $\mathrm{MgSO}_{4}$, filtered and concentrated under reduced pressure to give S3 (425 mg, 94\%) as a pale-yellow solid. This procedure was repeated three times to collect $1.29 \mathrm{~g}$ of the product. ${ }^{1} \mathrm{H}$ NMR $\left(600 \mathrm{MHz}, \mathrm{CDCl}_{3}\right) \delta 8.64(\mathrm{~d}, J=8.9 \mathrm{~Hz}, 2 \mathrm{H})$, $8.55(\mathrm{~s}, 2 \mathrm{H}), 7.94(\mathrm{~d}, J=8.9 \mathrm{~Hz}, 2 \mathrm{H}), 7.87(\mathrm{~d}, J=7.9 \mathrm{~Hz}, 2 \mathrm{H}), 7.45(\mathrm{dd}, J=8.1,1.2 \mathrm{~Hz}, 2 \mathrm{H}), 2.65$ (s, 6H). ${ }^{13} \mathrm{C}$ NMR $\left(151 \mathrm{MHz}, \mathrm{CDCl}_{3}\right) \delta 136.3,130.7,130.2,128.3,128.2,128.0,126.8,122.7,120.3$, 22.3. HRMS $\left(\mathrm{APCl}^{+}\right) \mathrm{m} / \mathrm{z}$ calcd for $\mathrm{C}_{20} \mathrm{H}_{17}[\mathrm{M}+\mathrm{H}]^{+}:$: 257.1325, found 257.1325.

\section{3,9-Bis(bromomethyl)chrysene (2)}

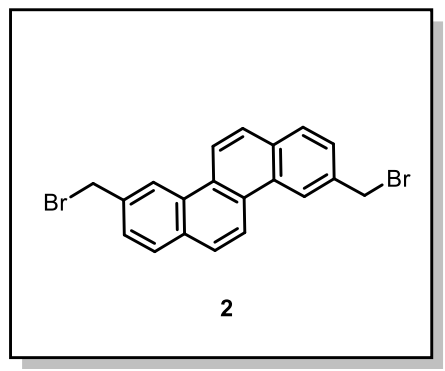

To a solution of $\mathbf{S} 3(1.24 \mathrm{~g}, 4.84 \mathrm{mmol})$ in $\mathrm{CCl}_{4}(100 \mathrm{~mL})$ were added $\mathrm{N}$ bromosuccinimide (1.89 g, $10.6 \mathrm{mmol}, 2.2$ equiv) and benzoyl peroxide $(116 \mathrm{mg}, 0.48 \mathrm{mmol}, 9.9 \mathrm{~mol} \%)$. After stirring the resulting mixture at $80^{\circ} \mathrm{C}$ for $3 \mathrm{~h}$, the solvent was evaporated and the resulting solid was washed with water, ethanol and diethyl ether, and recrystallized from ethanol to afford $2(1.54 \mathrm{~g}, 77 \%)$ as a white solid. ${ }^{1} \mathrm{H}$ NMR $(600 \mathrm{MHz}$, $\left.\mathrm{Cl}_{2} \mathrm{CHCHCl}_{2}, 80{ }^{\circ} \mathrm{C}\right) \delta 8.80(\mathrm{~s}, 2 \mathrm{H}), 8.75(\mathrm{~d}, J=8.9 \mathrm{~Hz}, 2 \mathrm{H}), 8.05(\mathrm{~d}, J$ $=9.3 \mathrm{~Hz}, 2 \mathrm{H}), 8.03(\mathrm{~d}, J=8.6 \mathrm{~Hz}, 2 \mathrm{H}), 7.73(\mathrm{~d}, J=8.3 \mathrm{~Hz}, 2 \mathrm{H}), 4.86(\mathrm{~s}$, $4 \mathrm{H}) .{ }^{13} \mathrm{C}$ NMR $\left(151 \mathrm{MHz}, \mathrm{TCE}-\mathrm{d}_{2}, 80^{\circ} \mathrm{C}\right) \delta 136.1,131.9,130.3,129.2$, 128.3, 127.3, 127.1, 123.4, 121.6, 34.2. HRMS (direct El ${ }^{+}$at $200{ }^{\circ} \mathrm{C}$ ) $\mathrm{m} / \mathrm{z}$ calcd for $\mathrm{C}_{20} \mathrm{H}_{14} \mathrm{Br}_{2}\left[\mathrm{M}^{+}\right.$ (monoisotopic): 411.9462 , found: 411.9457. 
(Naphthalene-2,6-diylbis(methylene))bis(triphenylphosphonium) bromide (S4).

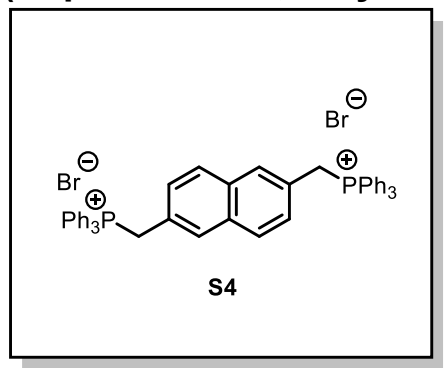

S4 was synthesized according to the literature procedure. ${ }^{21} \mathrm{H}$ NMR $(600$ $\left.\mathrm{MHz}, \mathrm{DMSO}-\mathrm{d}_{6}\right) \delta 7.93-7.88(\mathrm{~m}, 6 \mathrm{H}), 7.75-7.67(\mathrm{~m}, 24 \mathrm{H}), 7.48(\mathrm{~d}, \mathrm{~J}=$ $8.6 \mathrm{~Hz}, 2 \mathrm{H}), 7.45$ (br s, 2H), $7.05(\mathrm{~d}, J=8.6 \mathrm{~Hz}, 2 \mathrm{H}), 5.39$ (d, J = 15.8 $\mathrm{Hz}, 4 \mathrm{H}) .{ }^{13} \mathrm{C}$ NMR $\left(151 \mathrm{MHz}, \mathrm{DMSO}-\mathrm{d}_{6}\right) \delta 135.1,134.0\left(\mathrm{~d},{ }^{2} J_{\mathrm{PC}}=10.1\right.$ $\mathrm{Hz}), 131.6,130.07\left(\mathrm{~d},{ }^{3} J_{\mathrm{PC}}=13.0 \mathrm{~Hz}\right), 128.7,128.0,126.5,126.4,117.7$ $\left(\mathrm{d},{ }^{1} \mathrm{~J}_{\mathrm{PC}}=85.3 \mathrm{~Hz}\right), 28.2\left(\mathrm{~d},{ }^{1} \mathrm{~J}_{\mathrm{PC}}=4.80 \mathrm{~Hz}\right)$. Spectral properties were in agreement with those previously reported.

\section{2,6-Bis(2-bromo-4-methylstyryl)naphthalene (S5)}

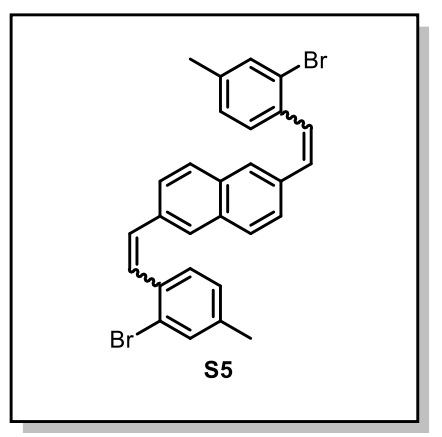

To a solution of 2-bromo-4-methylbenzaldehyde $(3.58 \mathrm{~g}, 18.0 \mathrm{mmol})$ and S4 $(6.83 \mathrm{~g}, 8.14 \mathrm{mmol})$ in dry THF $(600 \mathrm{~mL})$ was added a solution of potassium tert-butoxide $(2.24 \mathrm{~g}, 20.0 \mathrm{mmol})$ in $30 \mathrm{~mL}$ of dry THF dropwise through a syringe at $0{ }^{\circ} \mathrm{C}$ for $30 \mathrm{~min}$. After stirring the resulting mixture at room temperature for $13 \mathrm{~h}$, the mixture was quenched with $100 \mathrm{~mL}$ of $2 \mathrm{M} \mathrm{HCl}$ and diluted with ethyl acetate. Two layers were separated, and aqueous phase was extracted with AcOEt $(3 \times 75 \mathrm{~mL})$. Combined organic layers were concentrated under reduced pressure. The crude product was passed through a short pad of silica gel $\left(\mathrm{CHCl}_{3}\right)$ and purified by a flash column chromatography (hexane $/ \mathrm{CH}_{2} \mathrm{Cl}_{2}=9: 1$ ) to afford $\mathbf{S 5}(3.80 \mathrm{~g}, 90 \%)$ as a mixture of isomers in a relative ratio of $(Z, Z)-\mathbf{S 5} /(E, Z)-\mathbf{S 5} /(E, E)-\mathbf{S 5}=$ 8:6:1. HRMS $\left(\mathrm{APCl}^{+}\right) \mathrm{m} / \mathrm{z}$ calcd for $\mathrm{C}_{28} \mathrm{H}_{23} \mathrm{Br}_{2}[\mathrm{M}+\mathrm{H}]^{+}: 517.0163$ (monoisotopic), found 517.0161. Isomers were separated by a gradient flash chromatography (hexane $/ \mathrm{CH}_{2} \mathrm{Cl}_{2}=99: 1-95: 5$ ).
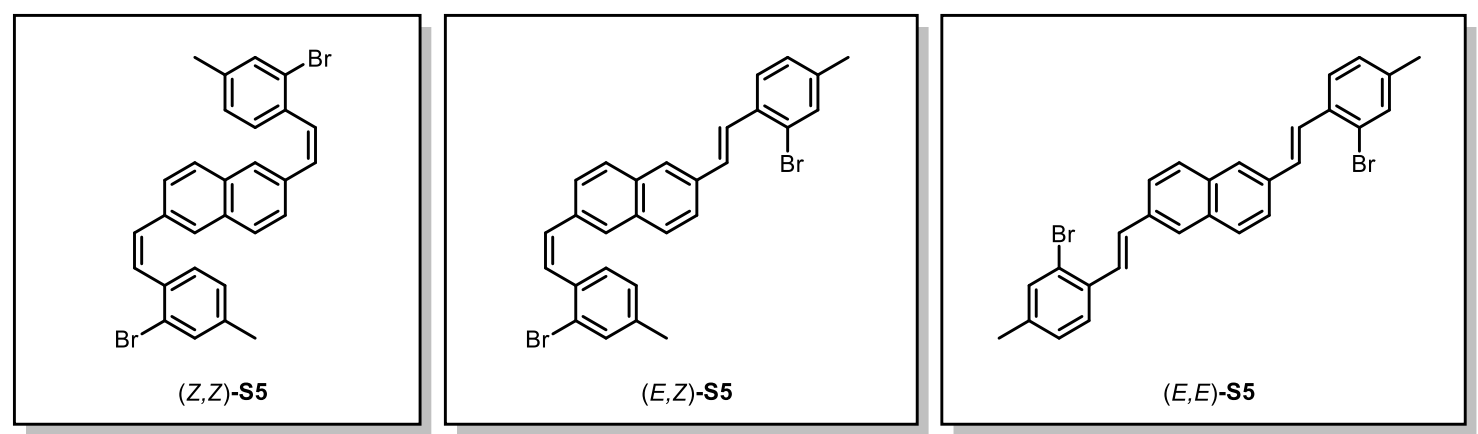

\section{2,6-Bis((Z)-2-bromo-4-methylstyryl)naphthalene ((Z,Z)-S5)}

White solid (non-fluorescent on TLC). ${ }^{1} \mathrm{H}$ NMR $\left(600 \mathrm{MHz}, \mathrm{CDCl}_{3}\right) \delta 7.56(\mathrm{~s}, 2 \mathrm{H}), 7.46-7.44(\mathrm{~m}, 4 \mathrm{H})$, $7.17(\mathrm{dd}, J=8.7,1.3 \mathrm{~Hz}, 2 \mathrm{H}), 7.08(\mathrm{~d}, J=7.8 \mathrm{~Hz}, 2 \mathrm{H}), 6.86(\mathrm{~d}, J=7.9,0.7 \mathrm{~Hz}, 2 \mathrm{H}), 6.76(\mathrm{~d}, J=12.4$ $\mathrm{Hz}, 2 \mathrm{H}), 6.65(\mathrm{~d}, J=12.4 \mathrm{~Hz}, 2 \mathrm{H}), 2.30(\mathrm{~s}, 6 \mathrm{H}) .{ }^{13} \mathrm{C}$ NMR $\left(151 \mathrm{MHz}, \mathrm{CDCl}_{3}\right) \delta 139.1,134.8,134.4$, $133.1,132.5,130.8,130.6,129.7,128.0,127.9,127.5,127.0,123.7,20.83$.

\section{2-((E)-2-Bromo-4-methylstyryl)-6-((Z)-2-bromo-4-methylstyryl)naphthalene ((E,Z)-S5)}

Yellow solid (fluorescent on TLC). ${ }^{1} \mathrm{H}$ NMR $\left(600 \mathrm{MHz}, \mathrm{CDCl}_{3}\right) \delta 7.75(\mathrm{~s}, 1 \mathrm{H}), 7.70$ (dd, $J=8.6,1.7$ $\mathrm{Hz}, 1 \mathrm{H}), 7.67(\mathrm{~d}, J=8.6 \mathrm{~Hz}, 1 \mathrm{H}), 7.63(\mathrm{~s}, 1 \mathrm{H}), 7.590(\mathrm{~d}, J=7.9 \mathrm{~Hz}, 1 \mathrm{H}), 7.585(\mathrm{~d}, J=8.6 \mathrm{~Hz}, 1 \mathrm{H})$, $7.53(\mathrm{~d}, J=16.2 \mathrm{~Hz}, 1 \mathrm{H}), 7.46(\mathrm{~d}, J=0.7 \mathrm{~Hz}, 1 \mathrm{H}), 7.42(\mathrm{~d}, J=0.7 \mathrm{~Hz}, 1 \mathrm{H}), 7.22(\mathrm{dd}, J=8.6,0.7 \mathrm{~Hz}$, $1 \mathrm{H}), 7.12(\mathrm{~d}, J=16.2 \mathrm{~Hz}, 1 \mathrm{H}), 7.13-7.11(\mathrm{~m}, 1 \mathrm{H}), 7.09(\mathrm{~d}, J=7.9 \mathrm{~Hz}, 1 \mathrm{H}), 6.86(\mathrm{dd}, J=7.7,1.0 \mathrm{~Hz}$, $1 \mathrm{H}), 6.79(\mathrm{~d}, J=12.0 \mathrm{~Hz}, 1 \mathrm{H}), 6.68(\mathrm{~d}, J=12.0 \mathrm{~Hz}, 1 \mathrm{H}), 2.33(\mathrm{~s}, 3 \mathrm{H}), 2.31(\mathrm{~s}, 3 \mathrm{H}) .{ }^{13} \mathrm{C}$ NMR $(151$ $\left.\mathrm{MHz}, \mathrm{CDCl}_{3}\right) \delta 139.2,139.1,134.9,134.8,134.5,134.2,133.5,133.14,133.07,132.8,130.8,130.6$, $130.4,129.8,128.5,128.4,128.2,127.9,127.64,127.58,127.3,126.6,126.3,124.0,123.9,123.8$, $20.84,20.80$. 
2,6-Bis((E)-2-bromo-4-methylstyryl)naphthalene ((E,E)-S5)

Bright yellow solid (fluorescent on TLC). ${ }^{1} \mathrm{H}$ NMR $\left(600 \mathrm{MHz}, \mathrm{CDCl}_{3}\right) \delta 7.84(\mathrm{~s}, 2 \mathrm{H}), 7.82(\mathrm{~d}, J=8.3$, $2 \mathrm{H}), 7.23(\mathrm{~d}, J=8.3 \mathrm{~Hz}, 2 \mathrm{H}), 7.76(\mathrm{dd}, J=8.6,1.4 \mathrm{~Hz}, 2 \mathrm{H}), 7.61(\mathrm{~d}, J=7.9 \mathrm{~Hz}, 2 \mathrm{H}), 7.56(\mathrm{~d}, J=$ $16.2 \mathrm{~Hz}, 2 \mathrm{H}), 7.45-7.43(\mathrm{~m}, 2 \mathrm{H}), 7.15(\mathrm{~d}, \mathrm{~J}=16.2 \mathrm{~Hz}, 2 \mathrm{H}), 7.15-7.13(\mathrm{~m}, 2 \mathrm{H}), 2.35(\mathrm{~s}, 6 \mathrm{H}) .{ }^{13} \mathrm{C}$ NMR $\left(151 \mathrm{MHz}, \mathrm{CDCl}_{3}\right) \delta 139.2,134.9,134.2,133.5,133.4,130.4,128.5(2 \mathrm{C}), 127.7,126.7,126.3,124.2$, 124.0, 20.8.

\section{1,9-Dibromo-3,11-dimethyldibenzo[c,I]chrysene (S6)}

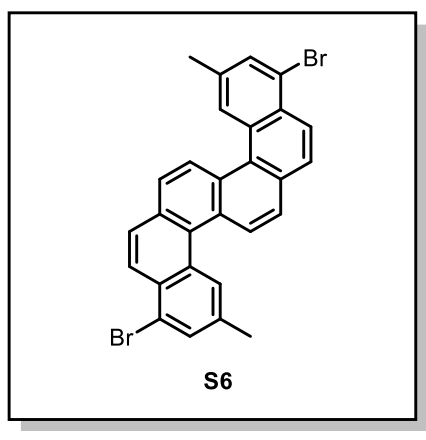

We utilized a strategy of bromine auxiliaries in photosynthesis of helicenes developed by Katz and co-workers. ${ }^{3}$ Argon was bubbled through a stirred solution of $\mathbf{S 5}$ (mixture of isomers) $(486 \mathrm{mg}, 0.94 \mathrm{mmol})$ and iodine $(1.02 \mathrm{~g}, 4.02 \mathrm{mmol})$ in dry benzene $(500 \mathrm{~mL})$ for $30 \mathrm{~min}$. Then, $3.0 \mathrm{~mL}$ of propylene oxide was added, and the mixture was irradiated with a $450 \mathrm{~W}$ UV lamp and stirred for additional $13 \mathrm{~h}$ at $30^{\circ} \mathrm{C}$. After the reaction was completed, the mixture was washed with aqueous $\mathrm{Na}_{2} \mathrm{~S}_{2} \mathrm{O}_{3}$ solution, water, and brine. The organic layer was dried with $\mathrm{MgSO}_{4}$, filtered and concentrated under reduced pressure to give $\mathbf{S 6}(463 \mathrm{mg}$, $96 \%)$ as a yellow solid. The procedure was repeated eight times to collect $3.70 \mathrm{~g}$ of the product. The corresponding photocyclization reactions using pure $(Z, Z)-\mathbf{S}$, $(E, Z)-\mathbf{S 5}$, and $(E, E)$-S5 gave $\mathbf{S 6}$ in $99 \%, 98 \%$ and $91 \%$ yields respectively. These products were used in the next step without further purifications. ${ }^{1} \mathrm{H} \mathrm{NMR}\left(600 \mathrm{MHz}, \mathrm{CDCl}_{3}\right) \delta 9.02(\mathrm{~d}, J=8.9 \mathrm{~Hz}$, $2 \mathrm{H}), 8.77(\mathrm{~s}, 2 \mathrm{H}), 8.37(\mathrm{~d}, J=8.9 \mathrm{~Hz}, 2 \mathrm{H}), 7.93(\mathrm{~d}, J=8.6 \mathrm{~Hz}, 2 \mathrm{H}), 7.92(\mathrm{~d}, J=8.6 \mathrm{~Hz}, 2 \mathrm{H}), 7.82(\mathrm{~s}$, $2 \mathrm{H}$ ), $2.62\left(\mathrm{~s}, 6 \mathrm{H}\right.$ ). HRMS (direct $\mathrm{El}^{+}$at $230{ }^{\circ} \mathrm{C}$ ) $\mathrm{m} / \mathrm{z}$ calcd for $\mathrm{C}_{28} \mathrm{H}_{18} \mathrm{Br}_{2}[\mathrm{M}+\mathrm{H}]^{+}: 511.9775$ (monoisotopic), found 511.9766 .

\section{3,11-Dimethyldibenzo[c,I]chrysene (S7)}

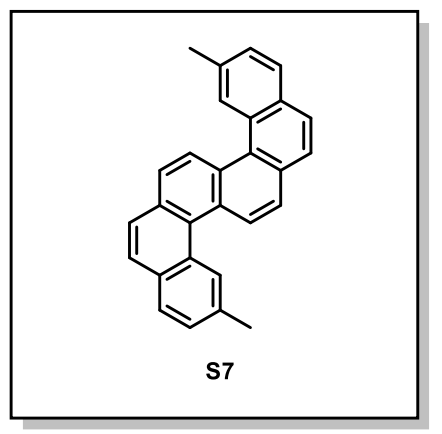

To a stirred solution of $\mathbf{S 6}(257 \mathrm{mg}, 0.50 \mathrm{mmol})$ in dry THF $(15 \mathrm{~mL})$ was added, a solution of $n$-butyllithium $(2.6 \mathrm{M}$ solution in hexane, $0.50 \mathrm{~mL}$, $1.30 \mathrm{mmol}, 2.6$ equiv) dropwise through a syringe at $-78^{\circ} \mathrm{C}$. After stirring for additional $30 \mathrm{~min}$, the resulting mixture was warmed to $0{ }^{\circ} \mathrm{C}$ over 30 min. The reaction was quenched with $1.5 \mathrm{~mL}$ water at $0{ }^{\circ} \mathrm{C}$, and the mixture was warmed to rt over $30 \mathrm{~min}$. Solvent was evaporated, and the crude product was passed through a short pad of silica gel $\left(\mathrm{CHCl}_{3}\right)$ giving S7 (169 $\mathrm{mg}, 95 \%)$ as a pale-yellow solid. A large-scale reaction using S6 $(3.68 \mathrm{~g}, 7.15 \mathrm{mmol})$ in dry THF $(200 \mathrm{~mL}), n$-butyllithium $(2.6 \mathrm{M}$ solution in hexane, $6.88 \mathrm{~mL}, 17.9 \mathrm{mmol}, 2.5$ equiv) and water $(2 \mathrm{~mL})$ gave $\mathbf{S} 7(2.25 \mathrm{~g}, 88 \%) .{ }^{1} \mathrm{H}$ NMR $\left(600 \mathrm{MHz}, \mathrm{CDCl}_{3}\right) \delta 9.11(\mathrm{~d}, \mathrm{~J}=8.6 \mathrm{~Hz}, 2 \mathrm{H}), 8.85(\mathrm{~s}, 2 \mathrm{H}), 7.95(\mathrm{~d}$, $J=8.3 \mathrm{~Hz}, 2 \mathrm{H}), 7.92(\mathrm{~d}, J=8.6 \mathrm{~Hz}, 2 \mathrm{H}), 7.90(\mathrm{~d}, J=8.6 \mathrm{~Hz}, 2 \mathrm{H}), 7.83(\mathrm{~d}, J=8.6 \mathrm{~Hz}, 2 \mathrm{H}), 7.48(\mathrm{dd}$, $J=8.1,1.2 \mathrm{~Hz}, 2 \mathrm{H}), 2.64(\mathrm{~s}, 6 \mathrm{H}) .{ }^{13} \mathrm{C}$ NMR $\left(151 \mathrm{MHz}, \mathrm{CDCl}_{3}\right) \delta 136.0,131.5,130.8,130.4,130.3$, $128.5,127.9,127.8,127.4,127.3,126.0,125.4,22.2$. One quaternary carbon peak can be overlapped. HRMS $\left(\mathrm{APCl}^{+}\right) \mathrm{m} / \mathrm{z}$ calcd for $\mathrm{C}_{28} \mathrm{H}_{21}[\mathrm{M}+\mathrm{H}]^{+}: 357.1638$, found 357.1632 . 


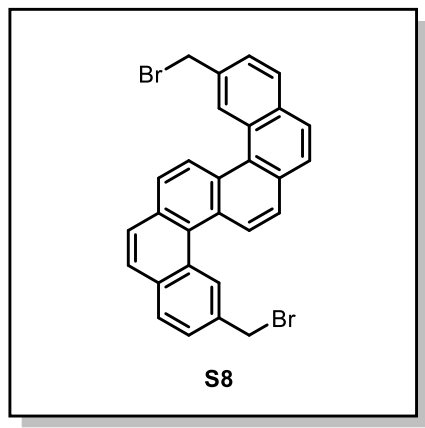

To a solution of $\mathbf{S} 7(2.31 \mathrm{~g}, 6.48 \mathrm{mmol})$ in $\mathrm{CCl}_{4}(120 \mathrm{~mL})$ were added $\mathrm{N}$ bromosuccinimide ( $2.67 \mathrm{~g}, 15.0 \mathrm{mmol}, 2.3$ equiv) and benzoyl peroxide $(157 \mathrm{mg}, 0.65 \mathrm{mmol}, 10 \mathrm{~mol} \%)$. After stirring the resulting mixture at $80{ }^{\circ} \mathrm{C}$ for $3 \mathrm{~h}$, the solvent was evaporated and the crude product was purified by a flash column chromatography (hexane $/ \mathrm{CH}_{2} \mathrm{Cl}_{2}=3: 1$ ) to afford $\mathbf{S 8}(2.20 \mathrm{~g}, 66 \%)$ as a yellow solid. ${ }^{1} \mathrm{H}$ NMR $\left(600 \mathrm{MHz}, \mathrm{CDCl}_{3}\right) \delta$ $9.08(\mathrm{~d}, J=8.6 \mathrm{~Hz}, 2 \mathrm{H}), 9.05-9.04(\mathrm{br} \mathrm{s}, 2 \mathrm{H}), 8.05(\mathrm{~d}, J=8.3 \mathrm{~Hz}, 2 \mathrm{H})$, $7.99(\mathrm{~d}, J=8.7 \mathrm{~Hz}, 2 \mathrm{H}), 7.95(\mathrm{~d}, J=8.6 \mathrm{~Hz}, 2 \mathrm{H}), 7.93(\mathrm{~d}, J=8.6 \mathrm{~Hz}, 2 \mathrm{H})$, $7.70(\mathrm{dd}, J=8.3,1.7 \mathrm{~Hz}, 2 \mathrm{H}), 4.81(\mathrm{~s}, 4 \mathrm{H}) .{ }^{13} \mathrm{C} \mathrm{NMR}\left(151 \mathrm{MHz}, \mathrm{CDCl}_{3}\right)$ $\delta 135.7,133.2,130.7,130.6,130.1,129.4,128.7,127.51,127.50,127.4$, $127.01,126.94,126.4,34.5$. One quaternary carbon peak can be overlapped. HRMS (direct $\mathrm{El}^{+}$at $200{ }^{\circ} \mathrm{C}$ ) $\mathrm{m} / \mathrm{z}$ calcd for $\mathrm{C}_{28} \mathrm{H}_{18} \mathrm{Br}_{2}[\mathrm{M}]^{+}: 511.9775$ (mono isotopic), found 511.9782 .

\section{Dibenzo[c,I]chrysene-3,11-diyldimethanethiol (3)}

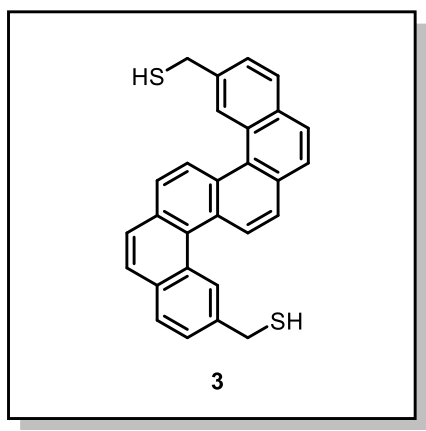

To a solution of S8 $(2.12 \mathrm{~g}, 4.12 \mathrm{mmol})$ in $300 \mathrm{~mL}$ of $1: 1$ mixture of acetone and $\mathrm{CHCl}_{3}$ was added thiourea (913 $\mathrm{mg}, 12.0 \mathrm{mmol}, 2.9$ equiv), and the resulting mixture was refluxed at $78{ }^{\circ} \mathrm{C}$ for $8 \mathrm{~h}$. The resulting thiouronium salt was collected by filtration and dissolved in $100 \mathrm{~mL}$ of $6 \% \mathrm{NaOH}$ solution and stirred at $80{ }^{\circ} \mathrm{C}$ for $2 \mathrm{~h}$. Then, the mixture was cooled to $0{ }^{\circ} \mathrm{C}$, and quenched with $6 \mathrm{M} \mathrm{HCl}$. The resulting solid was collected by filtration and washed with water and diethyl ether giving 3 $(1.40 \mathrm{~g}, 81 \%)$ as a yellow solid. ${ }^{1} \mathrm{H}$ NMR $\left(600 \mathrm{MHz}, \mathrm{CDCl}_{3}\right) \delta 9.09(\mathrm{~d}, J$ $=8.6 \mathrm{~Hz}, 2 \mathrm{H}), 8.97(\mathrm{~s}, 2 \mathrm{H}), 8.02(\mathrm{~d}, J=8.3 \mathrm{~Hz}, 2 \mathrm{H}), 7.96(\mathrm{~d}, J=8.6 \mathrm{~Hz}$, $2 \mathrm{H}), 7.92(\mathrm{~d}, J=8.6 \mathrm{~Hz}, 2 \mathrm{H}), 7.89(\mathrm{~d}, J=8.6 \mathrm{~Hz}, 2 \mathrm{H}), 7.64(\mathrm{dd}, J=8.2$, $1.4 \mathrm{~Hz}, 2 \mathrm{H}), 4.04(\mathrm{~d}, J=7.6 \mathrm{~Hz}, 4 \mathrm{H}), 1.89(\mathrm{t}, J=7.6 \mathrm{~Hz}, 2 \mathrm{H}) .{ }^{13} \mathrm{C}$ NMR $\left(151 \mathrm{MHz}, \mathrm{CDCl}_{3}\right) \delta 139.1$, $132.5,130.7,130.5,130.2,129.2,127.5,127.42,127.39,127.33,126.4,126.3,29.7$. One aromatic carbon peak can be overlapped. HRMS (direct $\mathrm{El}^{+}$at $300^{\circ} \mathrm{C}$ ) $\mathrm{m} / \mathrm{z}$ calcd for $\mathrm{C}_{28} \mathrm{H}_{20} \mathrm{~S}_{2}[\mathrm{M}]^{+}: 420.1006$, found 420.1023.
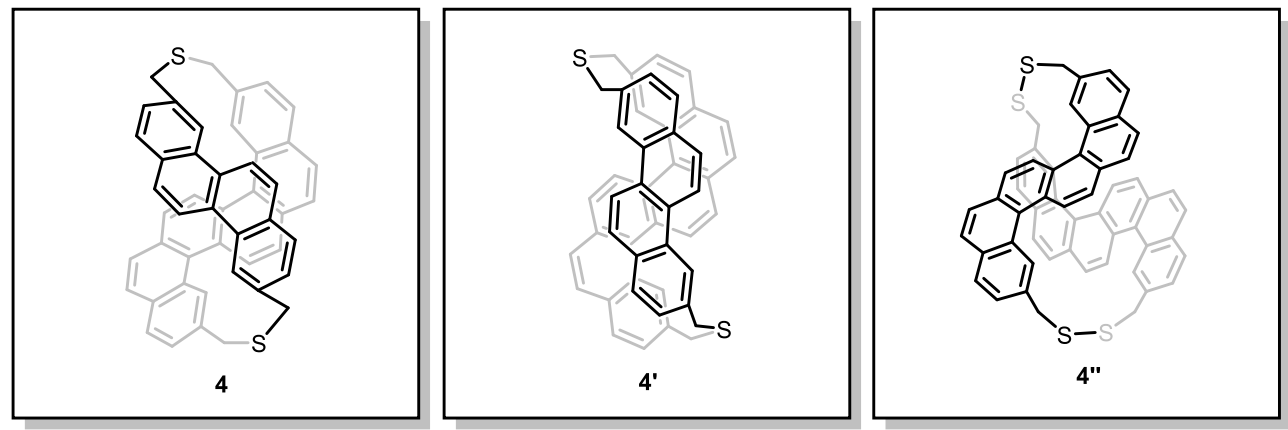

A solution of $2(1.36 \mathrm{~g}, 3.28 \mathrm{mmol})$ in dry DMF $(300 \mathrm{~mL})$ heated to $70{ }^{\circ} \mathrm{C}$ was slowly cannulated over $28 \mathrm{~h}$ to a stirred suspension of $\mathrm{Cs}_{2} \mathrm{CO}_{3}(1.18 \mathrm{~g}, 3.61 \mathrm{mmol})$ and $3(1.38 \mathrm{~g}, 3.28 \mathrm{mmol})$ in dry DMF $(500 \mathrm{~mL})$ heated to $55^{\circ} \mathrm{C}$. After stirring the mixture for additional $40 \mathrm{~h}$ at $55^{\circ} \mathrm{C}$, DMF was evaporated and residue was washed with water, ethanol and diethyl ether. Then the residue was washed with $\mathrm{CHCl}_{3}$ leaving pure 4 (550 mg, 25\%). Filtrate was subjected to a flash column chromatography (hexane/ $\mathrm{CH}_{2} \mathrm{Cl}_{2}=3: 1$ ) which allowed separation of the side products: conformer 4' (110 mg, 5\%) and disulfide dimer 4" (220 mg, 16\%).

Configuration of conformers were determined by ${ }^{1} \mathrm{H}$ NMR (Figure S1). In the case of conformer 4', three upfield-shifted signals (from 6.60 to $6.90 \mathrm{ppm}$ ) were observed in the spectrum, assigned to the hydrogen atoms indicated with colored spheres. Particularly indicative is doublet of doublets at 6.90 ppm marked with the red sphere. The corresponding hydrogen atom in $\mathbf{4}$ would not be affected by any benzene's electron cloud due to its spatial arrangement, hence no upfield shift would be observed. 


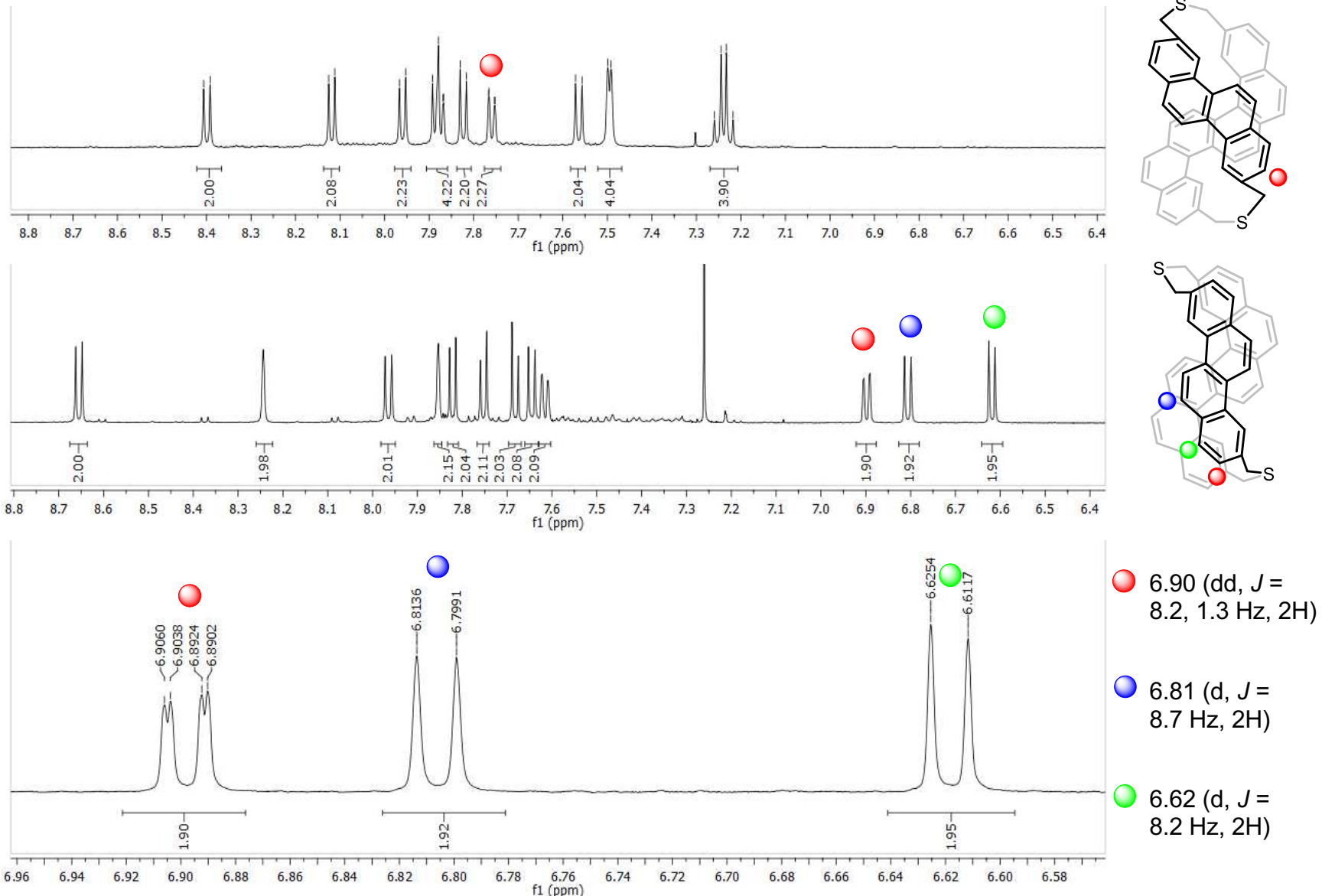

Figure S1. Zoomed-in aromatic region of ${ }^{1} \mathrm{H}$ NMR spectra of conformers 4 (top) and 4' (center and bottom).

3,7-Dithia-1(3,11)-dibenzo[c,I]chrysena-5(3,9)-chrysenacyclooctaphane (4). Yellow solid. ${ }^{1} \mathrm{H}$ NMR $\left(600 \mathrm{MHz}\right.$, TCE- $\left.d_{2}\right) \delta 8.40(\mathrm{~d}, J=8.7 \mathrm{~Hz}, 2 \mathrm{H}), 8.12(\mathrm{~d}, J=8.2 \mathrm{~Hz}, 2 \mathrm{H}), 7.96(\mathrm{~d}, J=8.4 \mathrm{~Hz}, 2 \mathrm{H})$, 7.90-7.86 (m, 4H), $7.82(\mathrm{~d}, J=8.4 \mathrm{~Hz}, 2 \mathrm{H}), 7.76(\mathrm{dd}, J=7.9,0.8 \mathrm{~Hz}, 2 \mathrm{H}), 7.56(\mathrm{~d}, J=8.8 \mathrm{~Hz}, 2 \mathrm{H})$, $7.50(\mathrm{~d}, J=4.4 \mathrm{~Hz}, 4 \mathrm{H}), 7.25(\mathrm{~d}, J=9.1 \mathrm{~Hz}, 2 \mathrm{H}), 7.23(\mathrm{~d}, J=9.1 \mathrm{~Hz}, 2 \mathrm{H}), 4.22(\mathrm{~d}, J=14.3 \mathrm{~Hz}, 2 \mathrm{H})$, $4.00(\mathrm{~d}, J=13.8 \mathrm{~Hz}, 2 \mathrm{H}), 3.86(\mathrm{~d}, J=13.8 \mathrm{~Hz}, 2 \mathrm{H}), 3.71(\mathrm{~d}, J=14.3 \mathrm{~Hz}, 2 \mathrm{H}) .{ }^{13} \mathrm{C}$ NMR $(151 \mathrm{MHz}$, TCE- $\left.d_{2}\right) \delta 135.6,134.7,131.9,130.5,130.2,129.75,129.67,129.1,128.9,128.7,128.2,127.1$, 127.0, 126.8, 126.7, 126.4, 126.3, 126.23, 126.16, 125.5, 124.0, 119.8, 35.66, 34.58. HRMS (APCl $\left.{ }^{+}\right)$ $\mathrm{m} / \mathrm{z}$ calcd for $\mathrm{C}_{48} \mathrm{H}_{33} \mathrm{~S}_{2}[\mathrm{M}+\mathrm{H}]^{+}: 673.2018$, found 673.2044 .

\section{3,7-Dithia-1(3,11)-dibenzo[c,]chrysena-5(3,9)-chrysenacyclooctaphane conformer (4')}

Yellow solid. ${ }^{1} \mathrm{H}$ NMR $\left(600 \mathrm{MHz}, \mathrm{CDCl}_{3}\right) \delta 8.66(\mathrm{~d}, \mathrm{~J}=8.7 \mathrm{~Hz}, 2 \mathrm{H}), 8.24(\mathrm{~s}, 2 \mathrm{H}), 7.96(\mathrm{~d}, \mathrm{~J}=8.9 \mathrm{~Hz}$, $2 \mathrm{H}), 7.85(\mathrm{~s}, 2 \mathrm{H}), 7.82(\mathrm{~d}, J=8.3 \mathrm{~Hz}, 2 \mathrm{H}), 7.75(\mathrm{~d}, J=8.3 \mathrm{~Hz}, 2 \mathrm{H}), 7.68(\mathrm{~d}, J=8.6 \mathrm{~Hz}, 2 \mathrm{H}), 7.64(\mathrm{~d}$, $J=8.9 \mathrm{~Hz}, 2 \mathrm{H}), 7.61(\mathrm{dd}, J=8.3,1.0 \mathrm{~Hz}, 2 \mathrm{H}), 6.89(\mathrm{dd}, J=8.3,1.4 \mathrm{~Hz}, 2 \mathrm{H}), 6.80(\mathrm{~d}, J=8.6 \mathrm{~Hz}$, $2 \mathrm{H}), 6.61(\mathrm{~d}, J=8.3 \mathrm{~Hz}, 2 \mathrm{H}), 4.31(\mathrm{~d}, J=15.1 \mathrm{~Hz}, 2 \mathrm{H}), 4.19(\mathrm{~d}, J=14.1 \mathrm{~Hz}, 2 \mathrm{H}), 4.03(\mathrm{~d}, J=15.1$ $\mathrm{Hz}, 2 \mathrm{H}), 3.99(\mathrm{~d}, J=14.1 \mathrm{~Hz}, 2 \mathrm{H}) .{ }^{13} \mathrm{C} N M R\left(151 \mathrm{MHz}, \mathrm{CDCl}_{3}\right) \delta 137.6,135.7,131.1,130.5,130.3$, $130.2,128.9,128.8,128.5,127.9,127.3,127.1,126.9,126.5,126.30,126.26,126.1,125.9,125.6$, 125.3, 122.5, 119.5, 39.4, 38.1. HRMS $\left(\mathrm{APCl}^{+}\right) \mathrm{m} / \mathrm{z}$ calcd for $\mathrm{C}_{48} \mathrm{H}_{33} \mathrm{~S}_{2}[\mathrm{M}+\mathrm{H}]^{+}: 673.2018$, found 673.2018.

\section{3,4,8,9-Tetrathia-1,6(3,11)-didibenzo[c,]chrysenacyclodecaphane (4")}

Yellow solid. ${ }^{1} \mathrm{H}$ NMR $\left(600 \mathrm{MHz}, \mathrm{CDCl}_{3}\right) \delta 8.39(\mathrm{~d}, J=7.9 \mathrm{~Hz}, 4 \mathrm{H}), 8.25(\mathrm{br} \mathrm{s}, 4 \mathrm{H}), 8.06(\mathrm{~d}, J=8.3$ $\mathrm{Hz}, 4 \mathrm{H}), 7.84(\mathrm{~d}, J=8.3 \mathrm{~Hz}, 4 \mathrm{H}), 7.74(\mathrm{~d}, J=8.3 \mathrm{~Hz}, 4 \mathrm{H}), 7.36(\mathrm{~d}, J=8.3 \mathrm{~Hz}, 4 \mathrm{H}), 6.55(\mathrm{~d}, J=7.3$ $\mathrm{Hz}, 4 \mathrm{H}), 4.10(\mathrm{~d}, J=12.7 \mathrm{~Hz}, 4 \mathrm{H}), 3.49(\mathrm{~d}, J=12.4 \mathrm{~Hz}, 4 \mathrm{H}) .{ }^{13} \mathrm{C}$ NMR $\left(151 \mathrm{MHz}, \mathrm{CDCl}_{3}\right) \delta 134.4$, $132.5,129.87,129.85,129.7,129.4,128.9,126.8,126.64,126.57,126.51,126.49,125.5,44.2$. HRMS $\left(\mathrm{APCl}^{+}\right) \mathrm{m} / \mathrm{z}$ calcd for $\mathrm{C}_{56} \mathrm{H}_{37} \mathrm{~S}_{4}[\mathrm{M}+\mathrm{H}]^{+}:$837.1773, found 837.1770. 


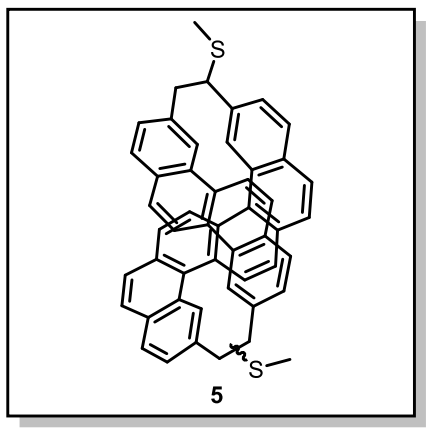

To a stirred solution of dithiacyclophane $4(67.3 \mathrm{mg}, 0.10 \mathrm{mmol})$ in dry $\mathrm{CH}_{2} \mathrm{Cl}_{2}(2 \mathrm{~mL})$ was added dimethoxycarbenium tetrafluoroborate (the Borch reagent) $\left(97.1 \mathrm{mg}, 0.60 \mathrm{mmol}\right.$ in $1 \mathrm{~mL}$ of $\mathrm{CH}_{2} \mathrm{Cl}_{2}$ ) dropwise at $0^{\circ} \mathrm{C}$. The resulting mixture was stirred at room temperature for $6 \mathrm{~h}$. Then, greenish-yellow solid was filtered and dried, giving intermediate salt in $95 \%(83.0 \mathrm{mg})$. The thus-obtained salt was subjected to the next step without further purification. A suspension of the salt $(83.0 \mathrm{mg})$ and 40.0 $\mathrm{mg}$ of $\mathrm{NaH}\left(60 \%\right.$ dispersion) in dry THF $(3 \mathrm{~mL})$ was stirred at $55^{\circ} \mathrm{C}$ for $48 \mathrm{~h}$ giving 5 as a mixture of stereoisomers $(40.5 \mathrm{mg}, 61 \%) .{ }^{1} \mathrm{H}$ and ${ }^{13} \mathrm{C}$ NMR $\left(600 \mathrm{MHz}, \mathrm{CDCl}_{3}\right.$ ): complex mixture due to the presence of regioisomers and diastereomers. HRMS $\left(\mathrm{ESI}^{+}\right) \mathrm{m} / \mathrm{z}$ calcd for $\mathrm{C}_{50} \mathrm{H}_{36} \mathrm{~S}_{2} \mathrm{Na}[\mathrm{M}+\mathrm{Na}]^{+}:$723.2151, found 723.2136. HRMS $\left(\mathrm{APCl}^{+}\right)$, molecular peak $\left(\mathrm{C}_{50} \mathrm{H}_{36} \mathrm{~S}_{2}[\mathrm{M}]^{+}: \mathrm{m} / \mathrm{z} 700\right)$ was not observed; $\mathrm{m} / \mathrm{z}$ calcd for $\mathrm{C}_{49} \mathrm{H}_{33} \mathrm{~S}\left[\mathrm{M}-\left(\mathrm{CH}_{3} \mathrm{SH}\right)+\mathrm{H}\right]^{+}:$653.2297, found 653.2298; m/z calcd for $\mathrm{C}_{48} \mathrm{H}_{29} \mathrm{~S}\left[\mathrm{M}-\left(\mathrm{CH}_{3} \mathrm{SH}\right)_{2}+\mathrm{H}\right]^{+}$: 605.2264, found 605.2263.

\section{2,6-Bis(methylsulfinyl)-1(3,11)-dibenzo[c,I]chrysena-4(3,9)-chrysenacyclohexaphane (6)}

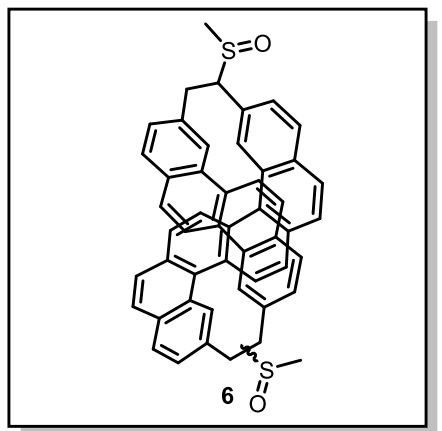

Cyclophane 5 (39.3 $\mathrm{mg}, 0.056 \mathrm{mmol})$ and 70\% $\mathrm{m}$-CPBA $(27.8 \mathrm{mg}$, $0.113 \mathrm{mmol})$ were dissolved in $\mathrm{CHCl}_{3}(4 \mathrm{~mL})$ at $0{ }^{\circ} \mathrm{C}$ and the resultant mixture was stirred at room temperature for $13 \mathrm{~h}$. Then, the solution was washed with $3 \% \mathrm{NaHCO}_{3}$ and dried. Solvent was evaporated giving pure $S, S^{\prime}$-bisoxide 6 in $99 \%$ yield $(40.8 \mathrm{mg})$. The thus-obtained product was subjected to the next step without further purification. ${ }^{1} \mathrm{H}$ and ${ }^{13} \mathrm{C}$ NMR $\left(600 \mathrm{MHz}, \mathrm{CDCl}_{3}\right)$ : complex mixture due to the presence of regioisomers and diastereomers. LRMS $\left(\mathrm{APCl}^{+}\right)$: molecular peak $\left(\mathrm{C}_{50} \mathrm{H}_{36} \mathrm{O}_{2} \mathrm{~S}_{2}[\mathrm{M}]^{+}: \mathrm{m} / \mathrm{z} 732\right)$ was not found; $\mathrm{m} / \mathrm{z}$ calcd for $\mathrm{C}_{49} \mathrm{H}_{33} \mathrm{OS}$ [M$\mathrm{CH}_{3} \mathrm{SO}^{+}:$669.2, found 669.2. HRMS $\left(\mathrm{APCl}^{+}\right), \mathrm{m} / \mathrm{z}$ calcd for $\mathrm{C}_{48} \mathrm{H}_{29}[\mathrm{M}-$ $\left.\left(\mathrm{CH}_{3} \mathrm{SOH}\right)_{2}+\mathrm{H}\right]^{+}: 605.2264$, found 605.2263 .

\section{(2Z,5Z)-1(3,11)-Dibenzo[c,I]chrysena-4(3,9)-chrysenacyclohexaphane-2,5-diene (7)}

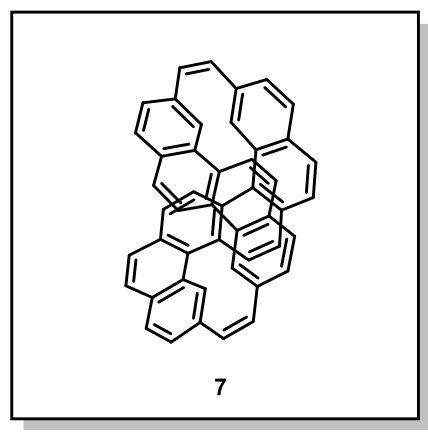

S,S-bisoxide $6(25.0 \mathrm{mg}, 0.034 \mathrm{mmol})$ was placed in a round-bottom flask immersed in a sand bath and directly connected to the oil pump through a glass joint. 6 was pyrolyzed at $500^{\circ} \mathrm{C}(0.04-0.05$ Torr) for $1 \mathrm{~h}$ to give intensively yellow pyrolysate which sublimed on the side of the flask. Pyrolysate was collected by washing the flask with $\mathrm{CHCl}_{3}$. Solvent was evaporated and the crude product was purified by a flash column chromatography (hexane/ $\left.\mathrm{CH}_{2} \mathrm{Cl}_{2}=95: 5\right)$ giving $7(9.5 \mathrm{mg}, 46 \%)$ as a yellow solid. ${ }^{1} \mathrm{H}$ NMR $\left(600 \mathrm{MHz}, \mathrm{CD}_{2} \mathrm{Cl}_{2}\right) \delta 8.82(\mathrm{~s}, 2 \mathrm{H}), 8.50(\mathrm{~s}, 2 \mathrm{H})$, $8.064(\mathrm{~d}, J=8.6 \mathrm{~Hz}, 2 \mathrm{H}), 8.058(\mathrm{~d}, J=8.3 \mathrm{~Hz}, 2 \mathrm{H}), 7.84(\mathrm{~d}, J=8.3 \mathrm{~Hz}$, $2 \mathrm{H}), 7.71(\mathrm{~d}, J=7.9 \mathrm{~Hz}, 2 \mathrm{H}), 7.64(\mathrm{~d}, J=8.6 \mathrm{~Hz}, 2 \mathrm{H}), 7.61$ (dd, $J=8.2$, $1.4 \mathrm{~Hz}, 2 \mathrm{H}), 7.49(\mathrm{dd}, J=8.0,0.8 \mathrm{~Hz}, 2 \mathrm{H}), 7.29(\mathrm{~d}, J=8.6 \mathrm{~Hz}, 2 \mathrm{H}), 7.13(\mathrm{~d}, J=8.9 \mathrm{~Hz}, 2 \mathrm{H}), 6.99(\mathrm{~d}$, $J=12.0 \mathrm{~Hz}, 2 \mathrm{H}), 6.93(\mathrm{~d}, J=8.9 \mathrm{~Hz}, 2 \mathrm{H}), 6.89(\mathrm{~d}, J=12.0 \mathrm{~Hz}, 2 \mathrm{H}) \cdot{ }^{13} \mathrm{C}$ NMR $\left(151 \mathrm{MHz}, \mathrm{CD}_{2} \mathrm{Cl}_{2}\right) \delta$ 134.4, 134.1, 133.1, 131.6, 131.4, 130.8, 130.4, 129.95, 129.89, 129.5, 129.1, 129.0, 128.7, 127.3, $127.23,127.20,127.0,126.74,126.71,126.1$ (2C), 125.4, 124.1, 120.0. Other two carbon peaks can be overlapped. HRMS (APCl ${ }^{+}$) $/ z$ calcd for $\mathrm{C}_{48} \mathrm{H}_{29}[\mathrm{M}+\mathrm{H}]^{+}: 605.2264$, found 605.2261. 


\section{Cyclo[c.c.c.c.c.c.e.e.e.e.e.e]dodecakisbenzene (infinitene, 1)}

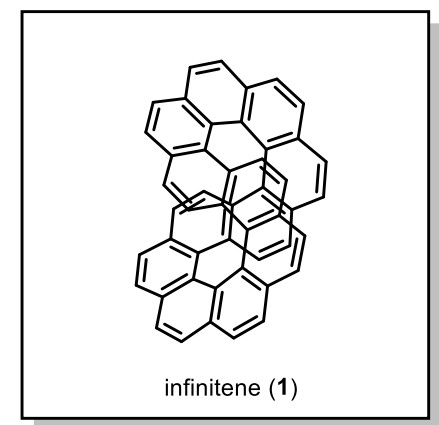

Argon was bubbled through a stirred solution of $7(9.0 \mathrm{mg}, 0.015 \mathrm{mmol})$ and iodine $(12.7 \mathrm{mg}, 0.050 \mathrm{mmol})$ in dry benzene $(10 \mathrm{~mL})$ for $10 \mathrm{~min}$. Then, $0.1 \mathrm{~mL}$ of propylene oxide was added, and the reaction mixture was irradiated with a $450 \mathrm{~W}$ UV lamp and stirred for additional $6 \mathrm{~h}$ at room temperature. After the reaction was completed, the mixture was washed with aqueous $\mathrm{Na}_{2} \mathrm{~S}_{2} \mathrm{O}_{3}$ solution, water, and brine. The organic layer was dried with $\mathrm{MgSO}_{4}$, filtered and concentrated under reduced pressure. The crude product was purified by a flash column chromatography (hexane $\left./ \mathrm{CH}_{2} \mathrm{Cl}_{2}=95: 5\right)$ to give infinitene $1(8.0 \mathrm{mg}$, $89 \%)$ as a yellow solid. ${ }^{1} \mathrm{H}$ NMR $\left(600 \mathrm{MHz}, \mathrm{CDCl}_{3}\right) \delta 8.18(\mathrm{~d}, J=7.9 \mathrm{~Hz}$, $4 \mathrm{H}), 8.16(\mathrm{~d}, J=7.9 \mathrm{~Hz}, 4 \mathrm{H}), 8.04(\mathrm{~d}, J=7.9 \mathrm{~Hz}, 4 \mathrm{H}), 7.60(\mathrm{~d}, J=8.0 \mathrm{~Hz}, 4 \mathrm{H}), 6.99(\mathrm{~d}, J=7.9 \mathrm{~Hz}$, $4 \mathrm{H}), 6.43(\mathrm{~d}, J=8.6 \mathrm{~Hz}, 4 \mathrm{H}) .{ }^{13} \mathrm{C}$ NMR $\left(151 \mathrm{MHz}, \mathrm{CDCl}_{3}\right) \delta 133.1,133.0,131.9,130.7,128.7,128.3$, $127.9,127.5,126.7,126.5,122.0,120.7$. One peak of quaternary carbon atom can be overlapped. HRMS $\left(\mathrm{APCl}^{+}\right) \mathrm{m} / \mathrm{z}$ calcd for $\mathrm{C}_{48} \mathrm{H}_{25}[\mathrm{M}+\mathrm{H}]^{+}: 601.1951$, found 601.1949 .

\section{X-ray crystallographic analysis}

Details of the crystal data and a summary of the intensity data collection parameters are listed in Table S1. A suitable crystal was mounted with mineral oil on a MiTeGen MicroMounts and transferred to the goniometer of the kappa goniometer of a RIGAKU XtaLAB Synergy-S system with $1.2 \mathrm{~kW}$ MicroMax-007HF microfocus rotating anode (Graphite-monochromated Mo K $\alpha$ radiation ( $\lambda$ $=0.71073 \AA)$ ) and PILATUS200K hybrid photon-counting detector. Cell parameters were determined and refined, and raw frame data were integrated using CrysAlisPro (Agilent Technologies, 2010). The structures were solved by direct methods with SHELXT ${ }^{4}$ and refined by full-matrix least-squares techniques against $F^{2}$ (SHELXL-2018/3) ${ }^{5}$ by using Olex2 software package. ${ }^{6}$ The intensities were corrected for Lorentz and polarization effects. The non-hydrogen atoms were refined anisotropically. Hydrogen atoms were placed using AFIX instructions. CCDC 2113525 contains the supplementary crystallographic data for this paper. These data can be obtained free of charge from The Cambridge Crystallographic Data Centre via www.ccdc.cam.ac.uk/data_request/cif. 
Table S1. Crystallographic data and structure refinement for 1.

\begin{tabular}{|c|c|c|}
\hline & \multicolumn{2}{|l|}{ Infinitene (1) } \\
\hline CCDC deposition No. & \multicolumn{2}{|l|}{2113525} \\
\hline Empirical formula & \multicolumn{2}{|l|}{$\mathrm{C}_{48} \mathrm{H}_{24}$} \\
\hline Formula weight & \multicolumn{2}{|l|}{600.67} \\
\hline Temperature & \multicolumn{2}{|l|}{$123(2) \mathrm{K}$} \\
\hline Wavelength & \multicolumn{2}{|l|}{$0.71073 \AA$} \\
\hline Crystal system & \multicolumn{2}{|l|}{ Monoclinic } \\
\hline Space group & \multicolumn{2}{|l|}{$P 2{ }_{1} / \mathrm{n}$} \\
\hline \multirow[t]{3}{*}{ Unit cell dimensions } & $a=11.2861(14) \AA$ & $\alpha=90^{\circ}$ \\
\hline & $b=13.3592(10) \AA$ & $\beta=102.261(11)^{\circ}$ \\
\hline & $c=19.508(2) \AA$ & $\gamma=90^{\circ}$ \\
\hline Volume & \multicolumn{2}{|l|}{$2874.2(5) \AA^{3}$} \\
\hline$Z$ & \multicolumn{2}{|l|}{4} \\
\hline Density (calculated) & \multicolumn{2}{|l|}{$1.388 \mathrm{~g} \cdot \mathrm{cm}^{-3}$} \\
\hline Absorption coefficient $(\mu)$ & \multicolumn{2}{|l|}{$0.079 \mathrm{~mm}^{-1}$} \\
\hline$F(000)$ & \multicolumn{2}{|l|}{1248.0} \\
\hline Crystal size & \multicolumn{2}{|c|}{$0.100 \times 0.100 \times 0.010 \mathrm{~mm}^{3}$} \\
\hline$\theta$ range for data collection & \multicolumn{2}{|l|}{$1.861-24.999^{\circ}$} \\
\hline Index ranges & \multicolumn{2}{|c|}{$-13 \leqq h \leqq 12,-15 \leqq k \leqq 15,-20 \leqq I \leqq 23$} \\
\hline Reflections collected & \multicolumn{2}{|l|}{16491} \\
\hline Independent reflections & \multicolumn{2}{|c|}{$5051[R$ (int) $=0.0586]$} \\
\hline Completeness to theta $=24.999^{\circ}$ & \multicolumn{2}{|l|}{$99.9 \%$} \\
\hline Refinement method & \multicolumn{2}{|c|}{ Full-matrix least-squares on $F^{2}$} \\
\hline Data / restraints / parameters & \multicolumn{2}{|l|}{$5051 / 0 / 433$} \\
\hline Goodness-of-fit on $F^{2}$ & \multicolumn{2}{|l|}{1.022} \\
\hline Final $\mathrm{R}$ indices $[I>2 \sigma(I)]$ & \multicolumn{2}{|c|}{$R_{1}=0.0639, w R_{2}=0.1497$} \\
\hline $\mathrm{R}$ indices (all data) & \multicolumn{2}{|c|}{$R_{1}=0.1485, w R_{2}=0.1924$} \\
\hline Extinction coefficient & \multicolumn{2}{|l|}{$\mathrm{n} / \mathrm{a}$} \\
\hline Largest diff. peak and hole & \multicolumn{2}{|c|}{0.793 and -0.223 e $\AA^{-3}$} \\
\hline
\end{tabular}




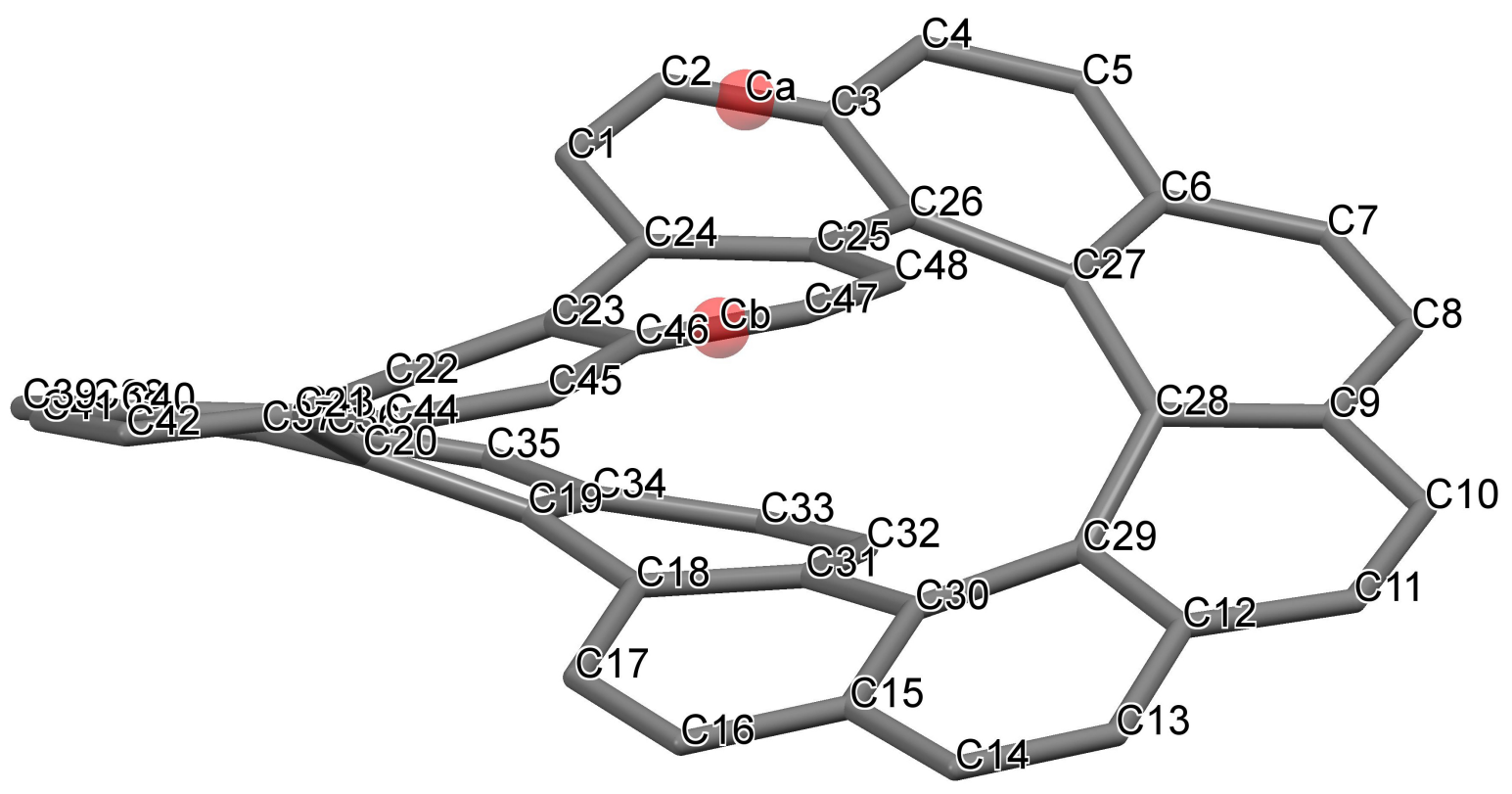

splay angle: $\phi_{\mathrm{torsion}}=\left(\phi_{\mathrm{C} 25-\mathrm{C} 26-\mathrm{C} 27-\mathrm{C} 28}+\phi_{\mathrm{C} 26-\mathrm{C} 27-\mathrm{C} 28-\mathrm{C} 29}+\phi_{\mathrm{C} 27-\mathrm{C} 28-\mathrm{C} 29-\mathrm{C} 30)} / 3=20.34^{\circ}\right.$ twist angle: $\phi_{\text {wist }}=(\phi c 2-\mathrm{Ca}-\mathrm{Cb}-\mathrm{C} 46)=22.03^{\circ}$

Figure S2. The molecular structure of 1 with labeled carbon atoms.

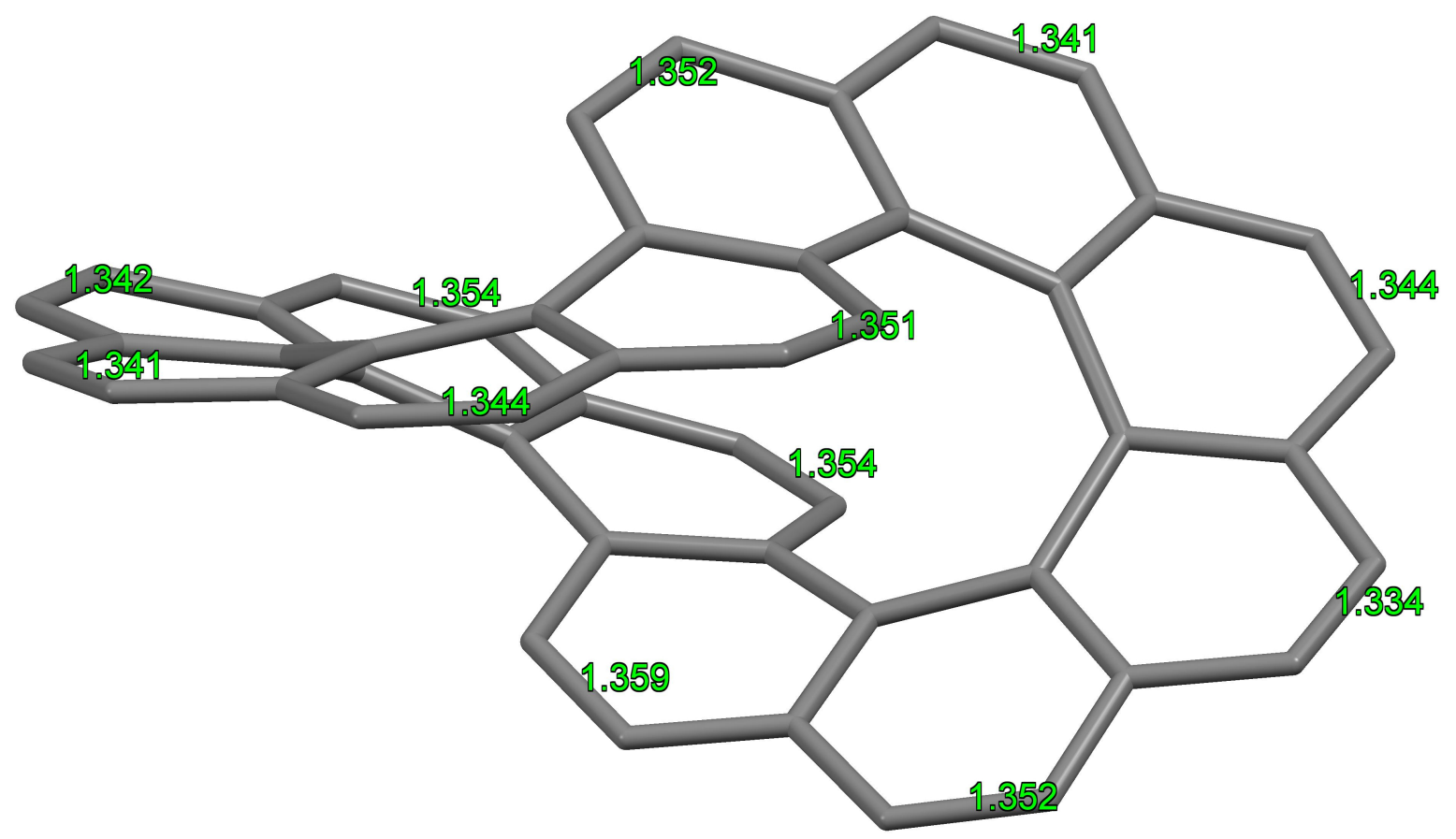

Figure S3. The molecular structure of 1 with listed bond lengths of the corresponding convex-armchair edges. 
(a)

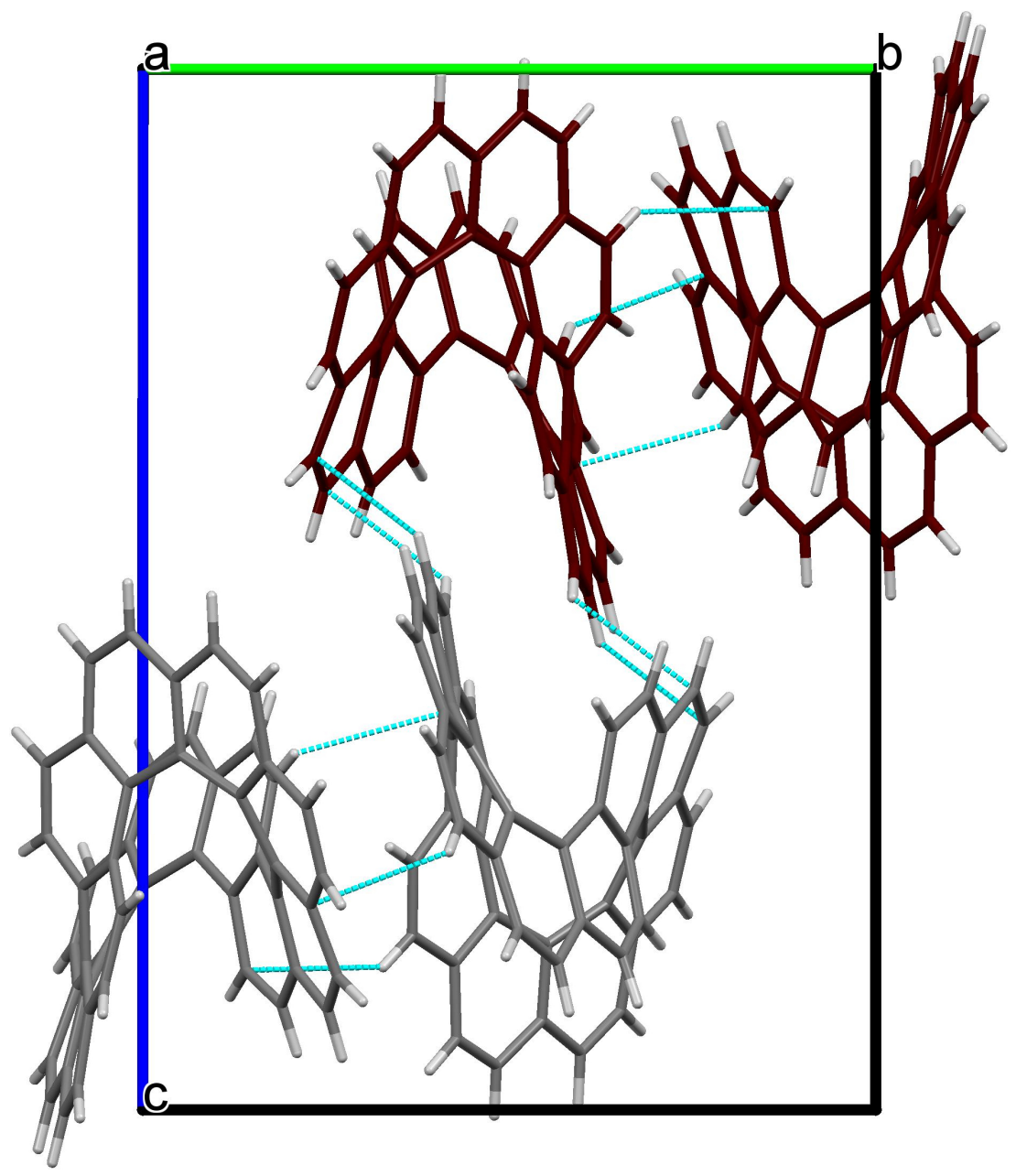

(b)

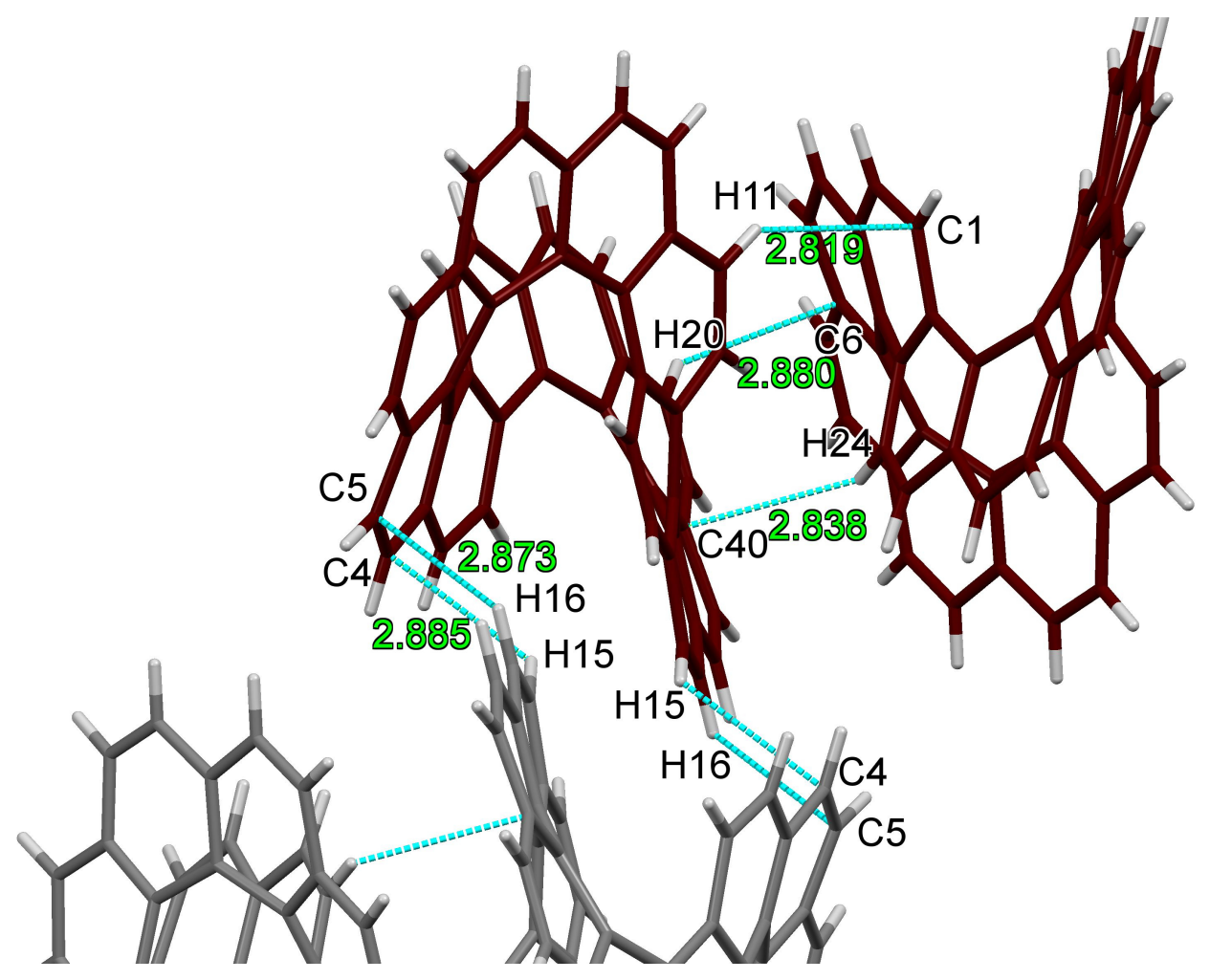

Figure S4. (a) The crystal packing viewed along the a-axis. (b) Zoomed-in view emphasizing short contacts. $(P, P)$-1 enantiomer colored in gray, $(M, M)$-1 enantiomer colored in red. 
(a)

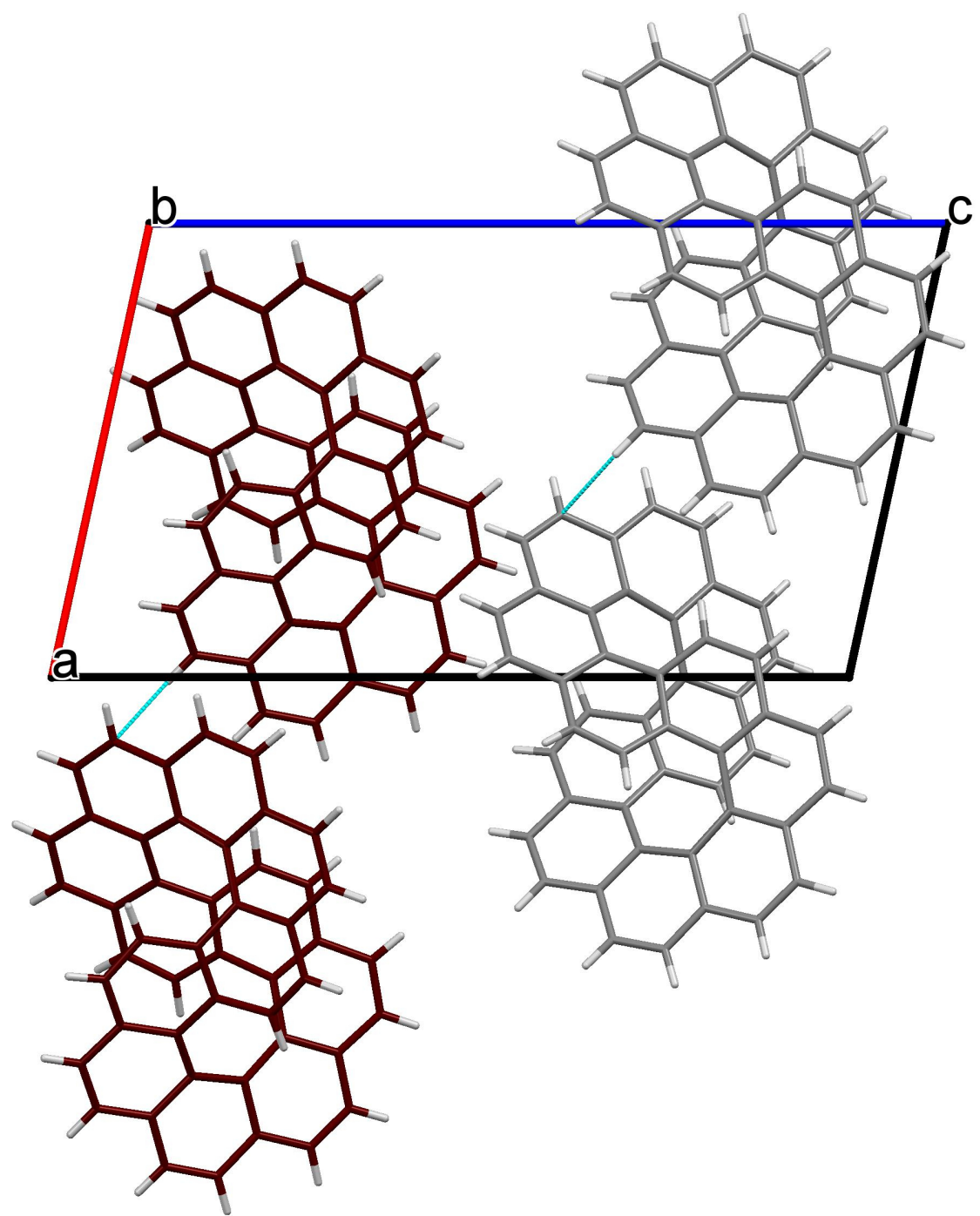

(b)

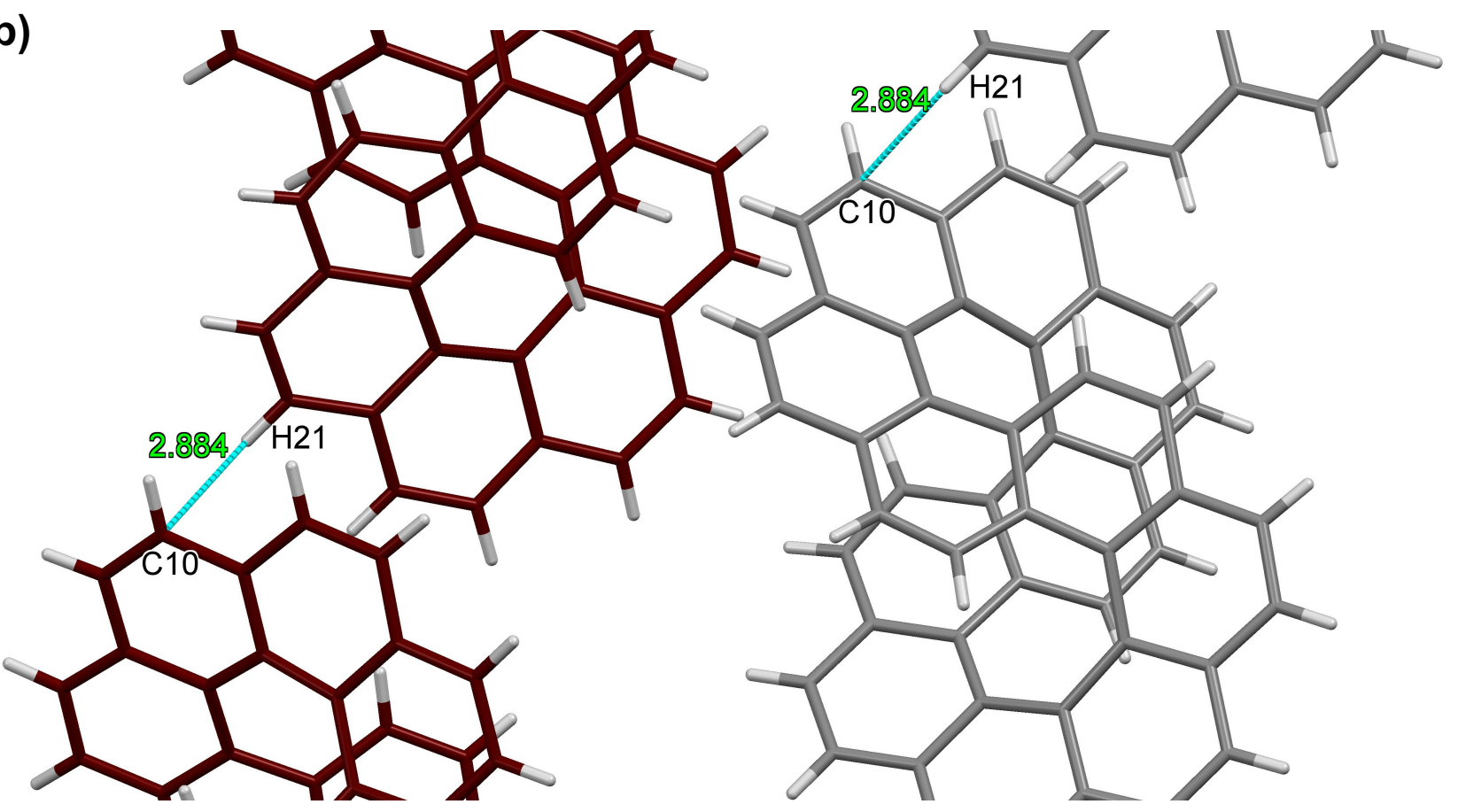

Figure S5. (a) The crystal packing viewed along the $b$-axis. (b) Zoomed-in view emphasizing short contacts. $(P, P)$-1 enantiomer colored in gray, $(M, M)$-1 enantiomer colored in red. 
(a)

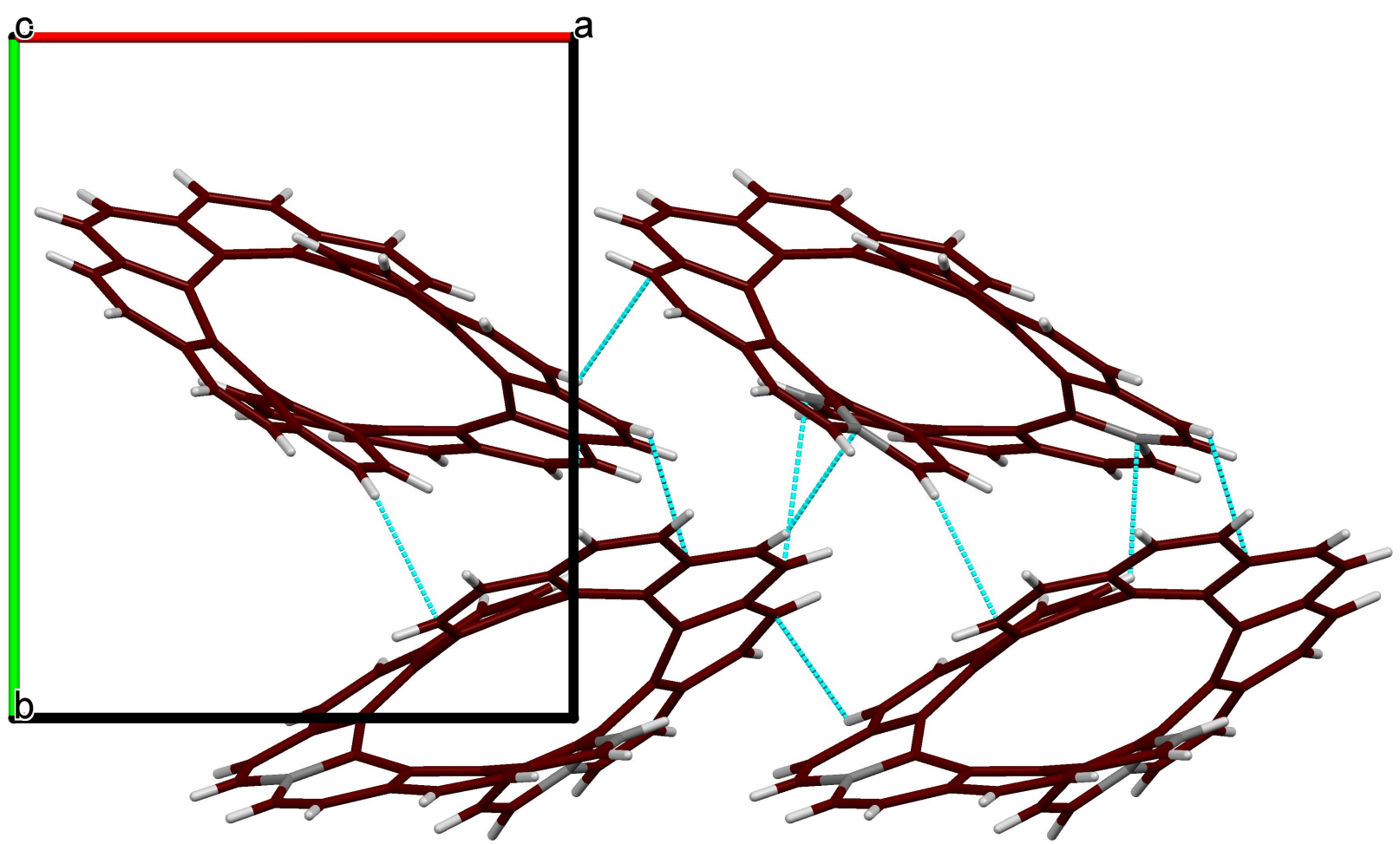

(b)

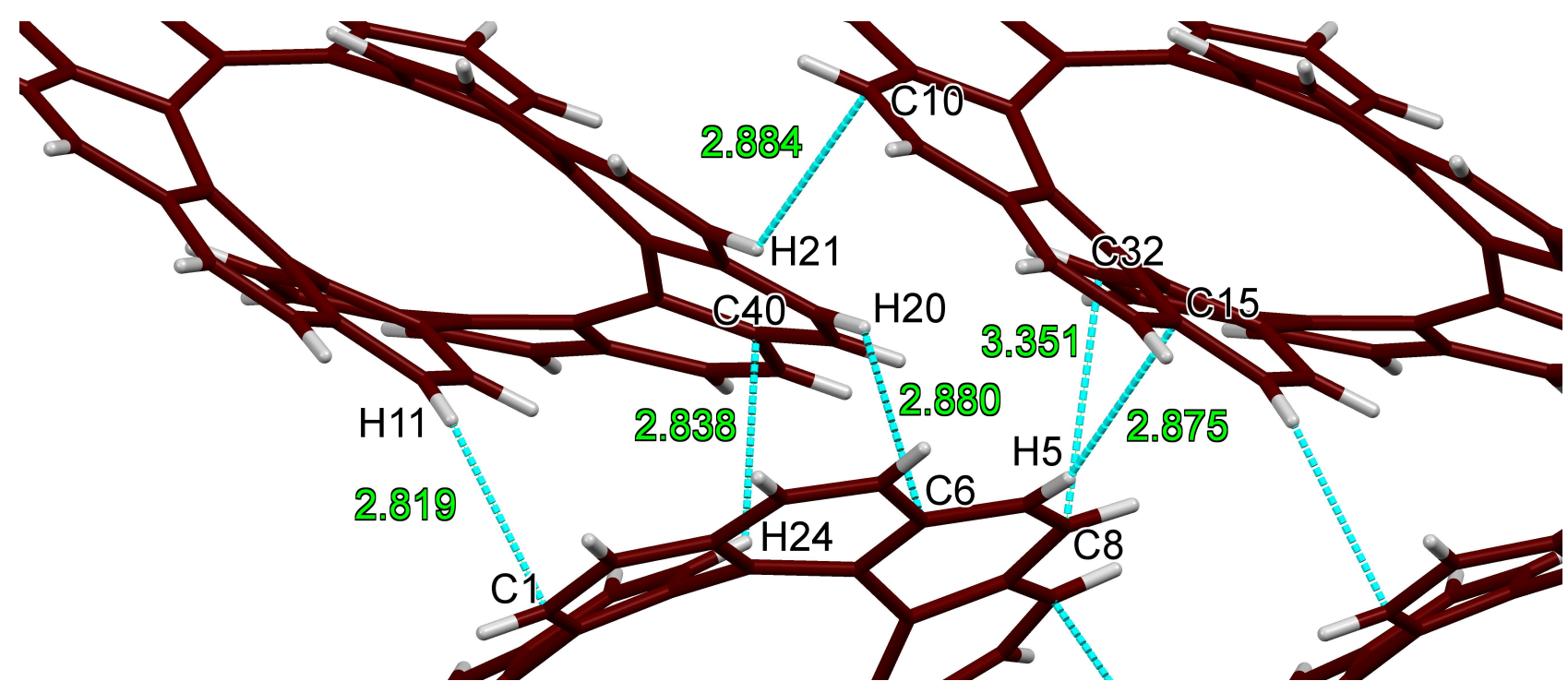

Figure S6. (a) The crystal packing viewed along the $c$-axis. (b) Zoomed-in view emphasizing short contacts. 


\section{Photophysical properties}

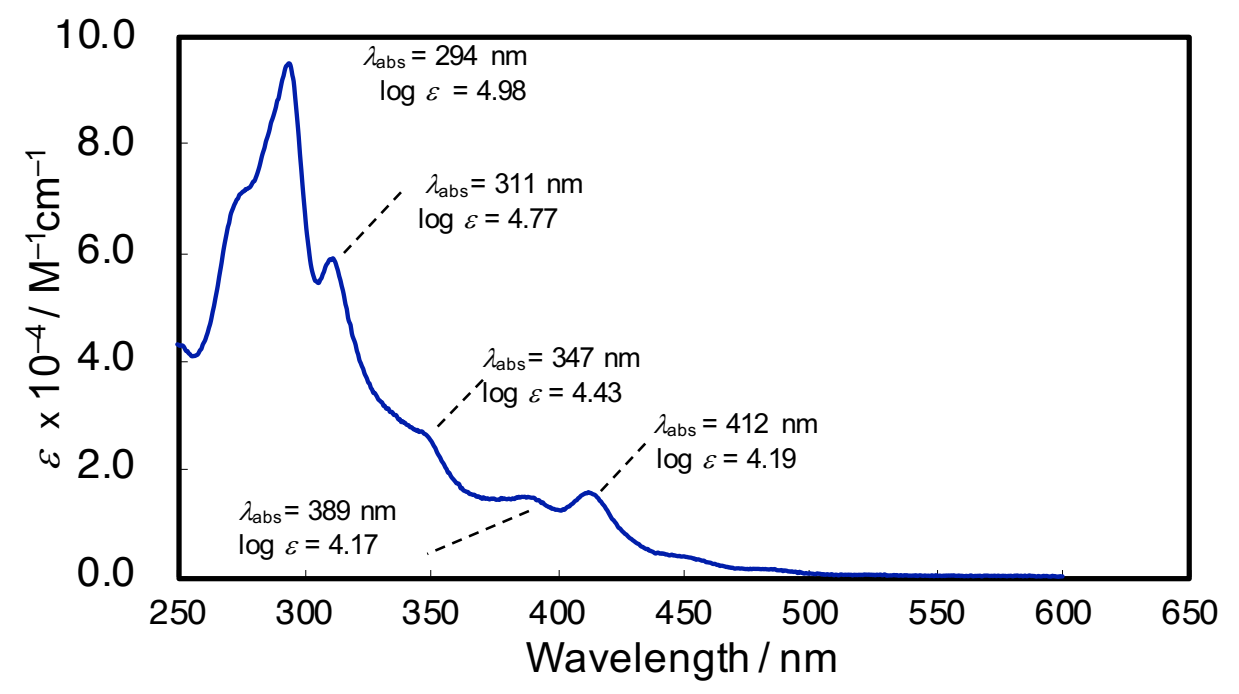

Figure S7. UV-vis absorption spectrum of 1 measured in $\mathrm{CH}_{2} \mathrm{Cl}_{2}$.

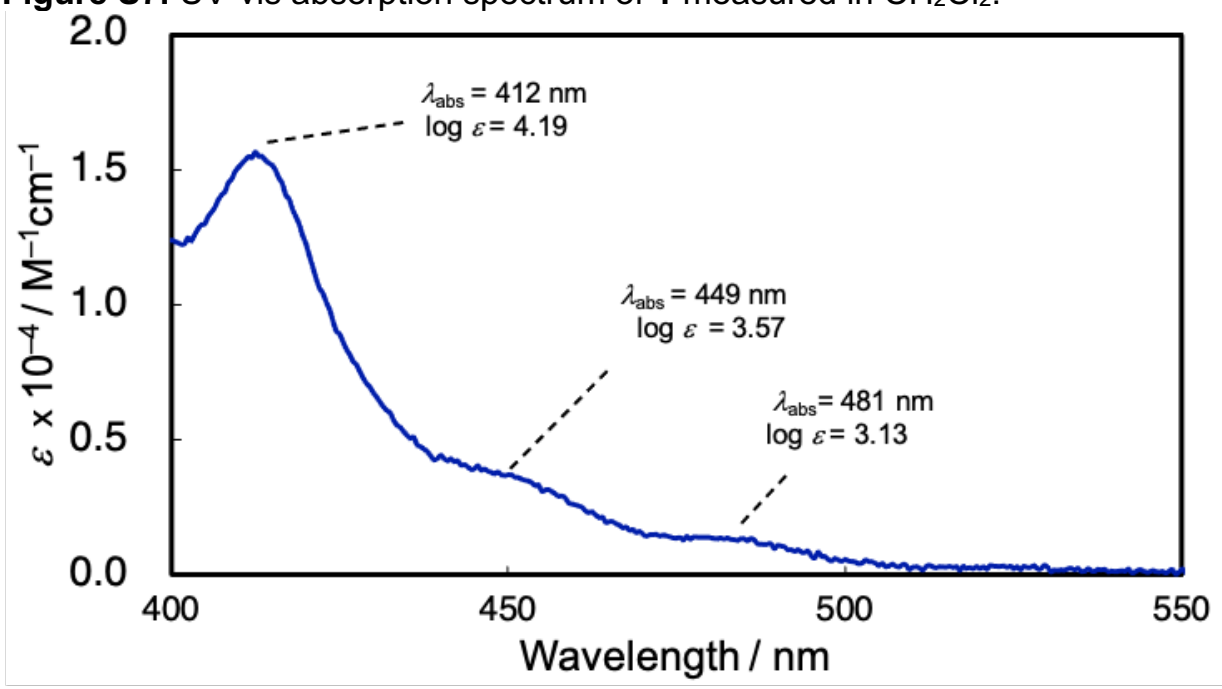

Figure S8. Zoomed-in region (400-550 nm) of absorption spectrum of 1.

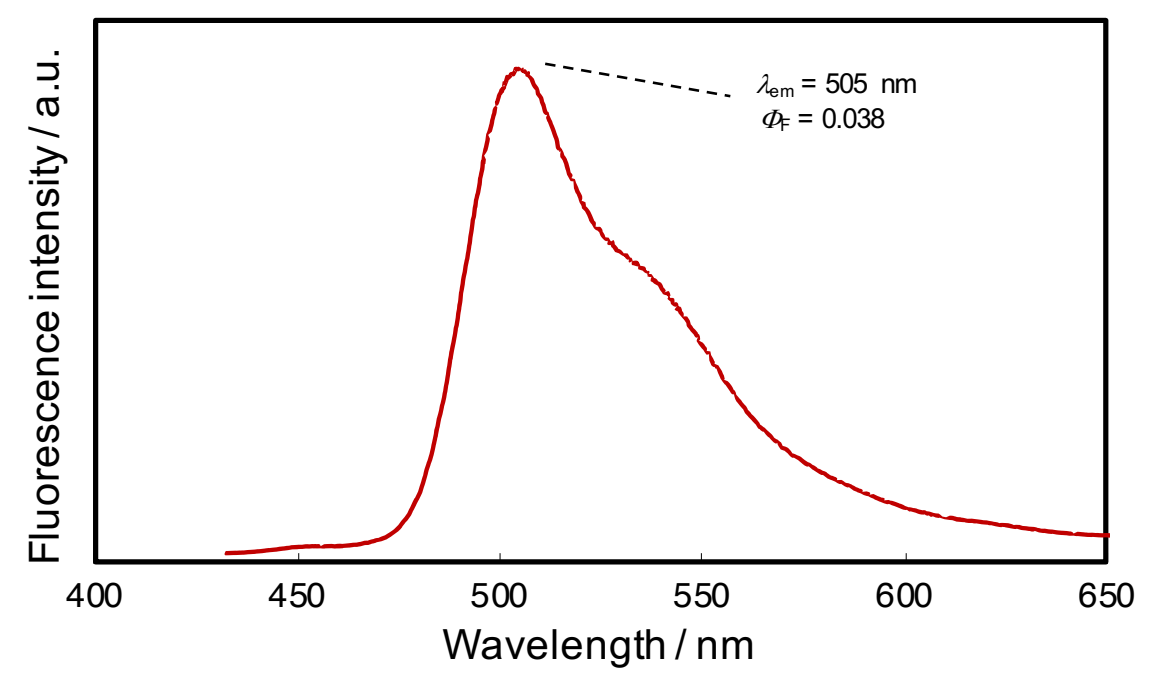

Figure S9. Emission spectrum of 1 measured in $\mathrm{CH}_{2} \mathrm{Cl}_{2}$. 


\section{Chiral HPLC analyses and CD spectra}

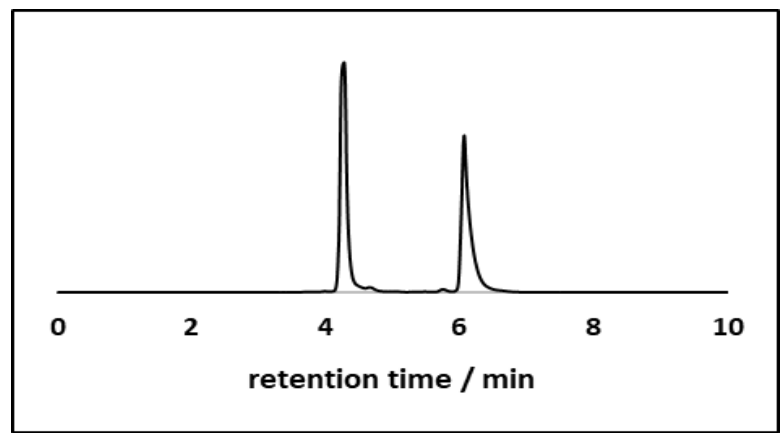

Figure S10. Chromatogram for the resolution of rac-1 using UV $(315 \mathrm{~nm})$ detectors in $n$-hexane/ $\mathrm{CH}_{2} \mathrm{Cl}_{2}(35: 65$, $\mathrm{v} / \mathrm{v})$ at the flow rate of $1.0 \mathrm{~mL} / \mathrm{min}$. Optical resolution was carried out with a DAICEL CHIRALPAK ${ }^{\circledR}$ IE column (0.46(i.d.) $\times 25 \mathrm{~cm}$ ) at $30^{\circ} \mathrm{C}$.

(a)

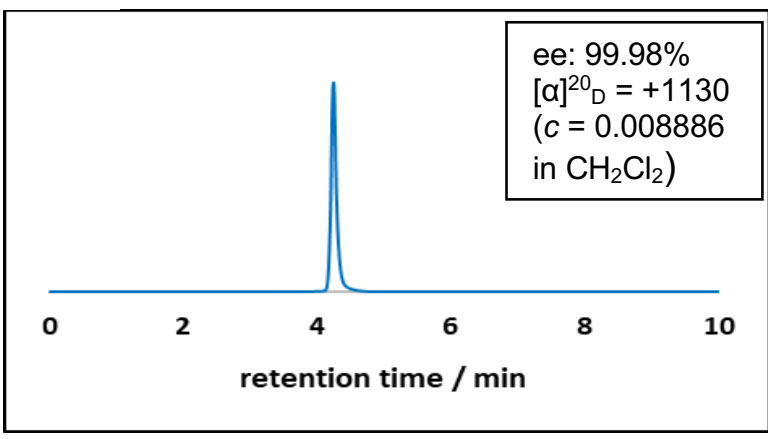

(b)

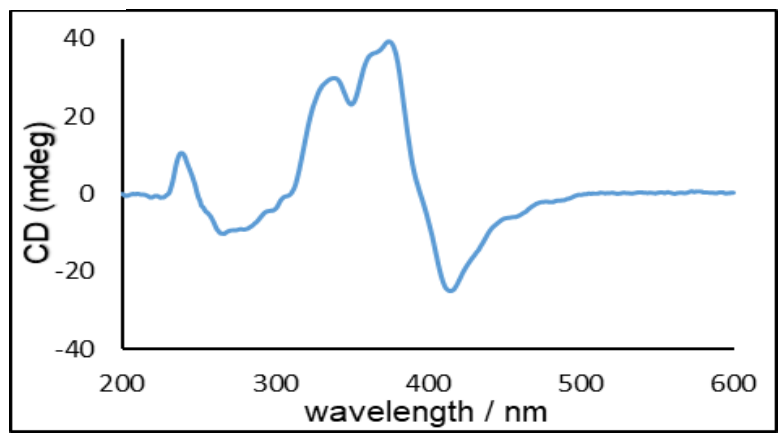

Figure S11. (a) Chromatogram of the eluted first fraction assigned as $(+)-(P, P)-1$. (b) CD spectrum of $(+)-$ $(P, P)-1$ measured in $\mathrm{CH}_{2} \mathrm{Cl}_{2}$.

(a)

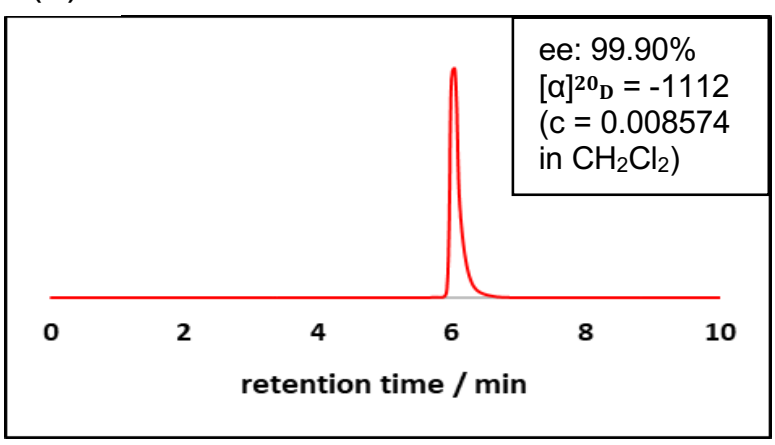

(b)

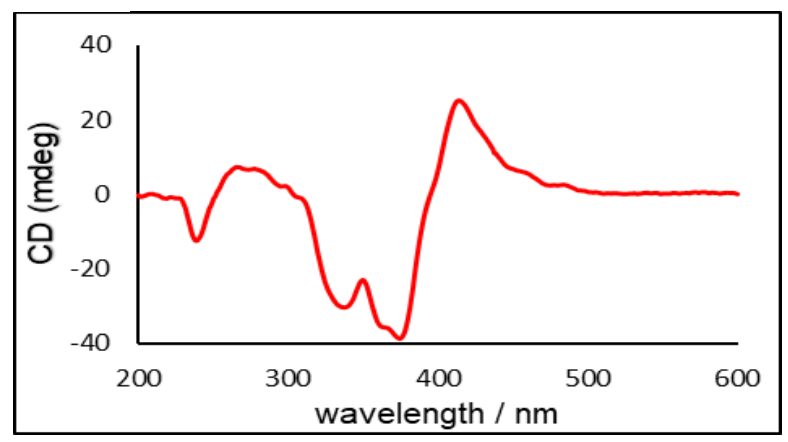

Figure S12. (a) Chromatogram of the eluted second fraction assigned as (-)-(M,M)-1. (b) CD spectrum of (-)$(M, M)-1$ measured in $\mathrm{CH}_{2} \mathrm{Cl}_{2}$.

Table S2. The anisotropy factors $\left|g_{\mathrm{CD}}\right|$ of selected transitions for $\mathbf{1}$.

\begin{tabular}{ccccccc} 
Wavelength $(\mathrm{nm})$ & 265 & 339 & 375 & 415 & 457 & 484 \\
$\varepsilon\left(\mathrm{M}^{-1} \mathrm{~cm}^{-1}\right)$ & 51549 & 28859 & 14485 & 15181 & 3021 & 1297 \\
$|\Delta \varepsilon|\left(\mathrm{M}^{-1} \mathrm{~cm}^{-1}\right)^{\mathrm{a}}$ & 17.82 & 52.48 & 68.97 & 43.78 & 10.36 & 3.04 \\
$\left|g_{\mathrm{CD}}\right|^{\mathrm{b}}$ & $3.5 \times 10^{-4}$ & $1.8 \times 10^{-3}$ & $4.8 \times 10^{-3}$ & $2.9 \times 10^{-3}$ & $3.4 \times 10^{-3}$ & $2.3 \times 10^{-3}$ \\
\hline
\end{tabular}

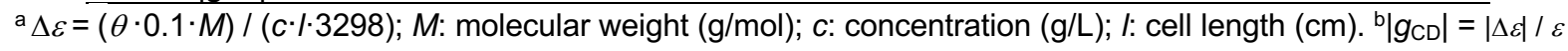




\section{Circularly polarized luminescence}

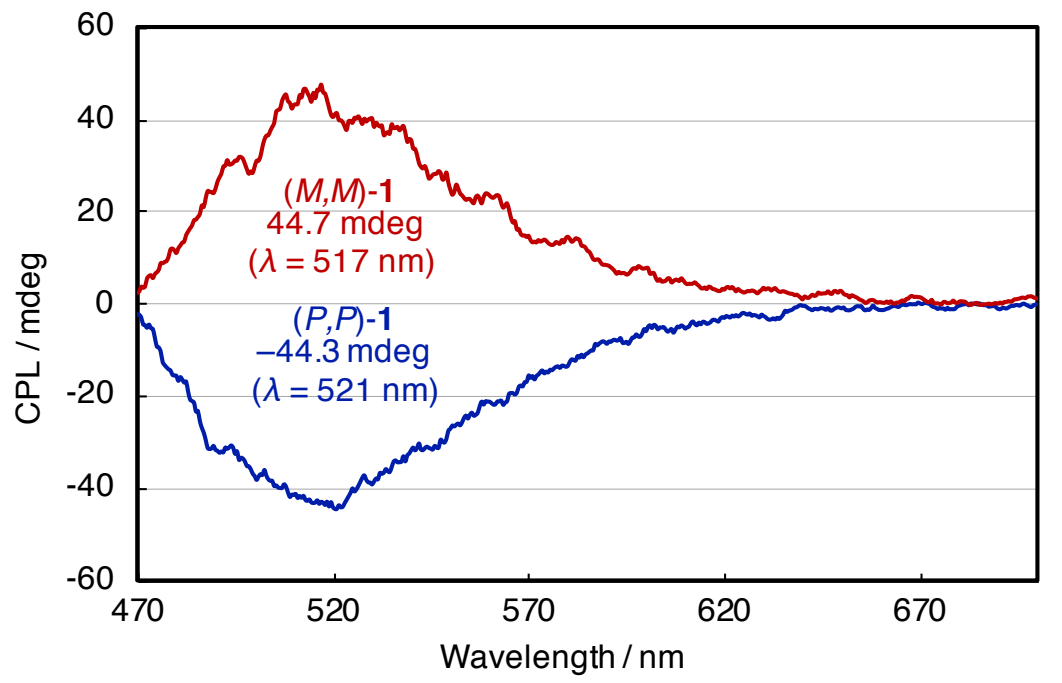

Figure S13. Circularly polarized luminescence (CPL) spectra of $(P, P)-1\left(c=2.4 \times 10^{-6} \mathrm{M}\right.$, blue line) and $(M, M)$ $1\left(2.9 \times 10^{-6} \mathrm{M}\right.$, red line $)$ in $\mathrm{CH}_{2} \mathrm{Cl}_{2}$ at $25^{\circ} \mathrm{C}$ with a $10 \times 10 \mathrm{~mm}$ cells. $\mathrm{CPL}=\Delta l=I_{L}-I_{R} ; I_{L}, I_{R}$ : intensities of left and right circularly polarized radiations. The spectra of dissymmetry factor were depicted in Figure 8 of the main text. $g_{\mathrm{CPL}}\left(g_{\text {lum }}\right)=(\Delta /) /(I)=(\Delta /) /\left[(1 / 2)\left(/ \mathrm{L}+/_{\mathrm{R}}\right)\right]$. 


\section{DFT calculations}

The computations were performed using Research Center for Computational Science, Okazaki, Japan. We carried out the computational studies by using the density functional theory (DFT) in Gaussian $16^{7}$ package (revision C. 01). All geometry optimizations of minima were conducted by using $P B E 0^{8} / 6-311+G(d, p)$ in gas phase at $273.15 \mathrm{~K}$. Frequency analyses were carried out at the same level to evaluate the zero-point vibrational energy and thermal corrections at 273.15. The nature of the stationary points was determined in each case according to the appropriate number of negative eigenvalues of the Hessian matrix. Calculations of single point energies, molecular orbitals and TD-DFT for all DFT-optimized structures were obtained by using the B3LYP $/ 6-311++G(d, p)$, and GIAO-NMR ${ }^{10}$ and NICS(0)/NICS $(1)^{11}$ were calculated using B3LYP/6-311+G(2d,p) with SMD $(\mathrm{CHCl} 3)$ model. ${ }^{12}$ Visualization of molecular orbitals was performed by the use of GaussView 6.0.16 software with 0.02 of isovalue. ${ }^{13}$

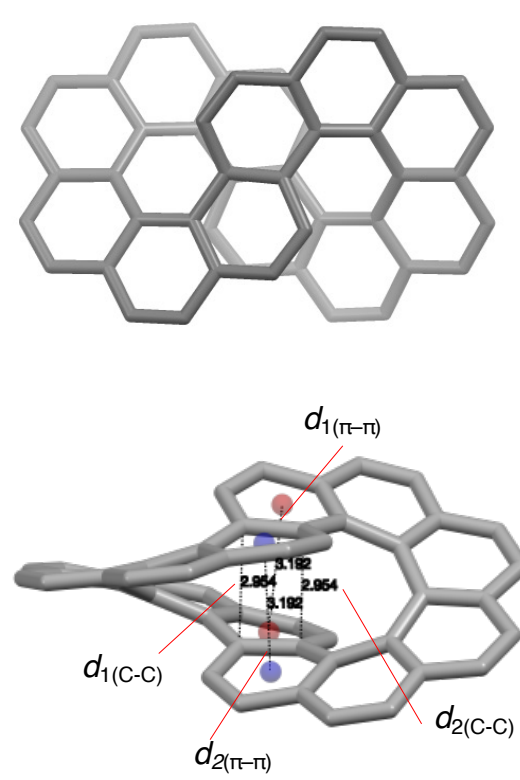

optimized structure by PBE0/6-311+G(d,p)

Red color: difference within $0.015 \AA$ Orange color: difference within $0.020 \AA$ : difference within $0.040 \AA$

\begin{tabular}{|c|c|c|c|c|c|}
\hline \multirow[b]{2}{*}{ Structure or basis sets } & \multicolumn{2}{|c|}{ Closest $\mathrm{C} \cdots \mathrm{C}$ distance } & \multicolumn{2}{|c|}{ Distance between centroids } & \multirow{2}{*}{$\begin{array}{l}\text { Cost } \\
\text { (day, hour) }\end{array}$} \\
\hline & $d_{1(\pi-\pi)}(\AA)$ & $d_{2(\mathrm{C}-\mathrm{C})}(\AA)$ & $d_{1(\pi-\pi)}(\AA)$ & $d_{2(\pi-\pi)}(\AA)$ & \\
\hline Experimental (X-ray) & 2.94166 & 2.91976 & 3.19245 & 3.15195 & \\
\hline B3LYP/6-31+G(d,p) & 3.00522 & & 3.26052 & & $2 \mathrm{~d} 17 \mathrm{~h}$ \\
\hline B3LYP/6-311+G(d,p) & 3.00195 & & - & & $4 d 15 h$ \\
\hline B3LYP/6-311+G(2d,p) & 3.00437 & & - & & $7 \mathrm{~d} 5 \mathrm{~h}$ \\
\hline B3LYP/6-311++G(2d,p) & 3.00450 & & - & & $8 \mathrm{~d} 0 \mathrm{~h}$ \\
\hline B3LYP/cc-pVDZ & 2.99892 & & - & & $9 \mathrm{~h}$ \\
\hline B3LYP/CC-PVTZ & 3.00565 & & - & & $6 \mathrm{~d} 16 \mathrm{~h}$ \\
\hline B3PW91/6-31+G(d,p) & 2.96991 & & - & & $2 \mathrm{~d} 18 \mathrm{~h}$ \\
\hline$M 06-2 X / 6-31+G(d, p)$ & 2.97353 & & - & & $3 \mathrm{~d} 7 \mathrm{~h}$ \\
\hline M06-2X/6-311+G(2d,p) & 2.96972 & & - & & 9d11h \\
\hline$B M K / 6-31 G(d)$ & 2.99800 & & - & & $3 \mathrm{~d} 2 \mathrm{~h}$ \\
\hline CAM-B3LYP/6-31+G(d,p) & 2.97869 & & - & & $1 \mathrm{~d} 23 \mathrm{~h}$ \\
\hline mPW1PW91/6-31+G(d,p) & 2.96071 & & 3.19917 & & $2 \mathrm{~d} 19 \mathrm{~h}$ \\
\hline PBE0/6-31G(d) & 2.95666 & & - & & 7h \\
\hline PBE0/6-31G(d,p) & 2.95682 & & - & & $8 \mathrm{~h}$ \\
\hline PBE0/6-311G(d,p) & 2.95316 & & 3.18465 & & $1 \mathrm{~d} 1 \mathrm{~h}$ \\
\hline $\mathrm{PBE} 0 / 6-31+\mathrm{G}(\mathrm{d})$ & 2.95955 & & - & & $2 \mathrm{~d} 2 \mathrm{~h}$ \\
\hline $\mathrm{PBE} 0 / 6-31+\mathrm{G}(\mathrm{d}, \mathrm{p})$ & 2.95992 & & - & & $2 \mathrm{~d} 20 \mathrm{~h}$ \\
\hline$P B E 0 / 6-311+G(d, p)$ & 2.95429 & & 3.19221 & & $4 \mathrm{~d} 21 \mathrm{~h}$ \\
\hline B3LYP-D3/6-31+G(d,p) & 3.00153 & & - & & $2 \mathrm{~d} 9 \mathrm{~h}$ \\
\hline B3LYP-D3/cc-PVDZ & 2.99795 & & - & & $11 \mathrm{~h}$ \\
\hline B3LYP-D3/cc-PVTZ & 3.00214 & & - & & $6 \mathrm{~d} 6 \mathrm{~h}$ \\
\hline M06-2X-D3/6-31+G(d,p) & 2.97532 & & 3.15530 & & $3 \mathrm{~d} 9 \mathrm{~h}$ \\
\hline M06-2X-D3/6-311+G(2d,p) & 2.97149 & & - & & $10 \mathrm{~d} 13 \mathrm{~h}$ \\
\hline BMK-D3/6-31+G(d,p) & & & - & & $2 \mathrm{~d} 18 \mathrm{~h}$ \\
\hline CAM-B3LYP-D3/6-31+G(d,p) & & & - & & $2 \mathrm{~d} 1 \mathrm{~h}$ \\
\hline B3PW91-D3/6-31+G(d,p) & & & - & & $2 \mathrm{~d} 19 \mathrm{~h}$ \\
\hline PBE0-D3/6-31G(d) & 2.96325 & & 3.15865 & & $7 \mathrm{~h}$ \\
\hline PBE0-D3/6-31G(d,p) & 2.96377 & & - & & $8 \mathrm{~h}$ \\
\hline PBE0-D3/6-31+G(d) & 2.96436 & & - & & $2 \mathrm{~d} 10 \mathrm{~h}$ \\
\hline PBE0-D3/6-31+G(d,p) & 2.96493 & & - & & $2 \mathrm{~d} 19 \mathrm{~h}$ \\
\hline PBE0-D3/6-311+G(d,p) & 2.96074 & & - & & $4 \mathrm{~d} 18 \mathrm{~h}$ \\
\hline
\end{tabular}

Figure S14. Comparison of basis sets for geometry optimization of inifintene (1). Calculation cost: job cpu time in Gaussian. 
Table S3. Uncorrected and thermal-corrected (298.15 K, $1 \mathrm{~atm})$ energies of stationary points (Hartree) calculated by PBE0/6-311+G(d,p). a

\begin{tabular}{ccccc}
\hline structure & $E$ & $E+Z P E$ & $H$ & $G$ \\
\hline$(P, P)-1$ & -1841.879574 & -1841.318388 & -1841.287702 & -1841.372361 \\
\hline benzene & -232.019728 & -231.918919 & -231.913580 & -231.946378 \\
\hline phenanthrene & -539.016349 & -538.821262 & -538.810864 & -538.856141
\end{tabular}

a) E: electronic energy; ZPE: zero-point energy; $H$ : sum of electronic and thermal correction to enthalpies; $G$ : sum of electronic and thermal correction to free energies.

Table S4. TD-DFT (B3LYP/6-311++(d,p)//PBE0/6-311+G(d,p)) vertical one-electron excitations (5 states) calculated for inifinitene (1).

\begin{tabular}{ccccc}
\hline Exited state & Energy / eV & Wavelength / nm & Oscillator strength & Description \\
\hline 1 & 2.5748 & 481.54 & 0.0001 & HOMO-1 to LUMO \\
2 & 2.5983 & 477.18 & 0.0113 & HOMO to LUMO+1 \\
3 & 2.7572 & 449.67 & 0.0024 & HOMO to LUMO+2 \\
4 & 2.8860 & 429.60 & 0.1226 & HOMO-1 to LUMO+2 \\
5 & 2.9834 & 415.58 & 0.0214 & HOMO-2 to LUMO+1 \\
\hline
\end{tabular}

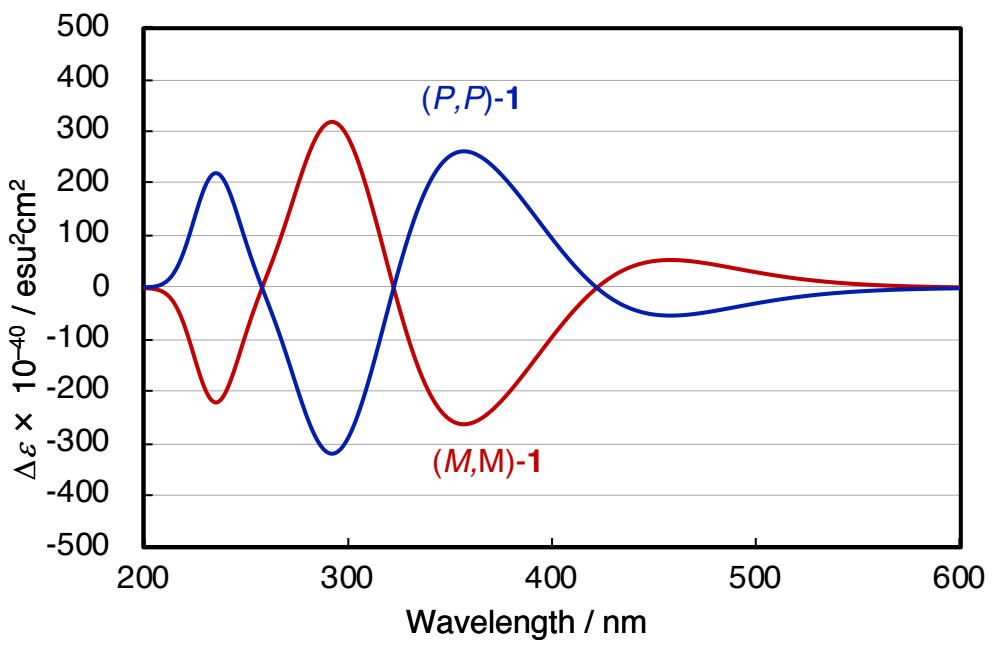

Figure S15. CD spectra of $(P, P)-1$ and $(M, M)-1$ simulated by TD-DFT (B3LYP/6-311++(d,p)//PBE0/6$311+\mathrm{G}(\mathrm{d}, \mathrm{p})$ with $N$ state $=100)$.

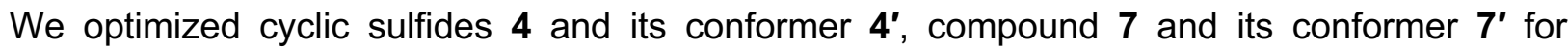
evaluating a possibility of interconversion and relative stabilities (Table S5). As a result, compounds 4 and 4' show almost the same stability, and weak intramolecular $\pi-\pi$ interactions (ca. 3.6-3.8 $\AA$ ) were observed (Table S5a). An activation energy from 4 to 4' are estimated to be $28.4 \mathrm{kcal} / \mathrm{mol}$ by PBE0/def2-TZVP//PBE0-D3/6-31G(d), which means that both compounds are even stable at room temperature but the interconversion can be possible at higher temperature. The structures of 7 and $\mathbf{7}^{\prime}$ are more strained compared to $\mathbf{4}$ and 4', which are rationalized by smaller sizes of macrocycles in 7 and $\mathbf{7}^{\prime}$ than 4 and ' 4'. Compound 7 was more stable than $\mathbf{7}^{\prime}$ by $6 \mathrm{kcal} / \mathrm{mol}$ (Table S5b). Interconversions corresponding to the rotation of a chrysene moiety or a dibenzochrysene moiety can be potentially expected, while any distinct transition states were not obtained due to those highly bent and strained structures. Therefore, we expected that not only the interconversion from 7 to $\mathbf{7}^{\prime}$ but also racemization would be difficult, and the optical resolution of enantiomers of 7 would also be possible. 
Table S5. DFT calculations for estimating relative stabilities of (a) $4 / 4^{\prime}$, (b) $7 / 7^{\prime}$, and the transition states of those interconversions.

(a) Interconversion of $\mathbf{4}$ to $\mathbf{4}^{\prime}$

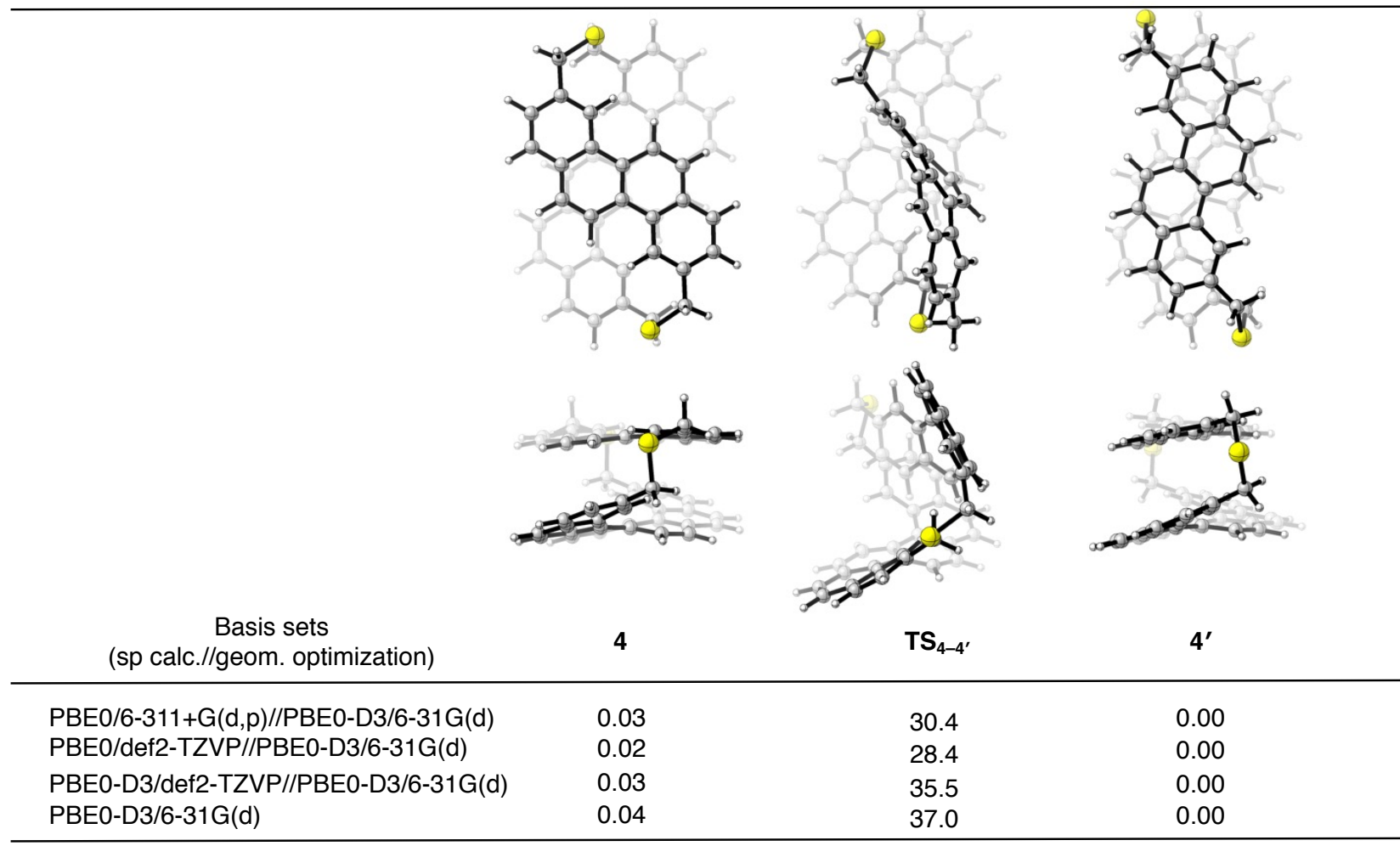

$\Delta G$ values $(\mathrm{kcal} / \mathrm{mol})$ are shown

(b) Optimizations of 7 and 7'
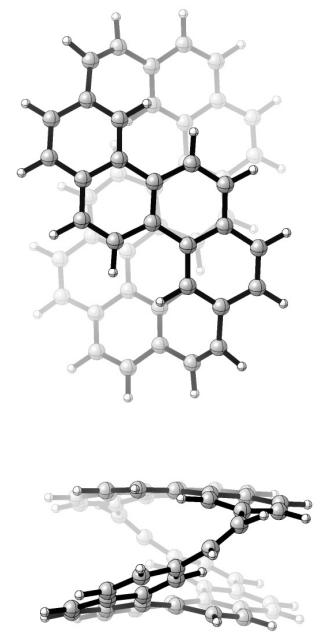

Basis sets

(sp calc.//geom. optimization)

PBE0/6-311+G(d,p)//PBE0-D3/6-31G(d) PBE0/def2-TZVP//PBE0-D3/6-31G(d)

PBE0-D3/def2-TZVP//PBE0-D3/6-31G(d)

PBE0-D3/6-31G(d)
7

0.00

0.00

0.00

0.00
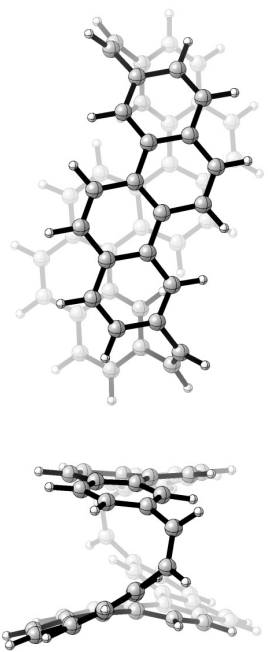

$\mathrm{TS}_{7-7^{\prime}}$

not located

not located

not located not located $7^{\prime}$

6.19

6.30

10.07

11.06 
Table S6. Uncorrected and thermal-corrected $(298.15 \mathrm{~K}, 1 \mathrm{~atm})$ energies of stationary points (Hartree) calculated by PBE0-D3/6-31G(d). ${ }^{\text {a }}$

\begin{tabular}{cccccccc}
\hline structure & $E$ & $E+Z P E$ & $H$ & $G$ & $\Delta_{Z P E}$ & $\Delta_{H}$ & $\Delta_{G}$ \\
\hline $\mathbf{4}$ & -2642.491233 & -2641.831783 & -2641.794579 & -2641.897272 & 0.659450 & 0.696655 & 0.593962 \\
\hline $\mathbf{4}^{\prime}$ & -2642.491236 & -2641.831809 & -2641.794598 & -2641.897332 & 0.659427 & 0.696638 & 0.593904 \\
\hline $\mathbf{T S}_{\mathbf{4 - 4}} \mathbf{4}^{\prime}$ & -2642.433001 & -2641.774315 & -2641.737887 & -2641.838408 & 0.658686 & 0.695114 & 0.594594 \\
\hline $\mathbf{7}$ & -1843.956216 & -1843.346127 & -1843.312921 & -1843.407502 & 0.610090 & 0.643296 & 0.548714 \\
\hline $\mathbf{7}^{\prime}$ & -1843.937261 & -1843.328625 & -1843.295247 & -1843.389885 & 0.608636 & 0.642014 & 0.547376 \\
\hline
\end{tabular}

a) $E$ : electronic energy; $Z P E$ : zero-point energy $(\triangle Z P E) ; H$ : sum of electronic and thermal correction to enthalpies $(E+$ $\left.\Delta_{H}\right)$; $G$ : sum of electronic and thermal correction to free energies $\left(E+\Delta_{G}\right) ; \Delta_{Z P E}$ : Zero-point energy correction; $\Delta_{H}$ : thermal correction to enthalpy; $\Delta_{G}$ : thermal correction to free energy.

Table S7. Single point calculations of electronic energies ( $E$ : Hartree) of each stationary points obtained by PBE0-D3/6-31G(d). ${ }^{\text {a }}$

\begin{tabular}{cccc}
\hline structure & $E(\mathrm{PBE} 0 / 6-311+\mathrm{G}(\mathrm{d}, \mathrm{p})$ & $E(\mathrm{PBE} / \mathrm{def2}-\mathrm{TZVP})$ & $E$ (PBE0-D3/def2-TZVP) \\
\hline $\mathbf{4}$ & -2642.842991 & -2643.052520 & -2643.137646 \\
\hline $\mathbf{4}^{\prime}$ & -2642.842976 & -2643.052501 & -2643.137641 \\
\hline $\mathbf{T S}_{4-\mathbf{4}^{\prime}}$ & -2642.795226 & -2643.007859 & -2643.081688 \\
\hline $\mathbf{7}$ & -1844.266608 & -1844.470821 & -1844.542359 \\
\hline $\mathbf{7}^{\prime}$ & -1844.255405 & -1844.459439 & -1844.524982 \\
\hline
\end{tabular}

a) $\quad E$ : electronic energy; $Z P E$ : zero-point energy $(\triangle Z P E)$; $H$ : sum of electronic and thermal correction to enthalpies (E + $\left.\Delta_{H}\right) ; G$ : sum of electronic and thermal correction to free energies $\left(E+\Delta_{G}\right) ; \Delta_{Z P E}$ : Zero-point energy correction; $\Delta_{H}$ : thermal correction to enthalpy; $\Delta_{G}$ : thermal correction to free energy.

Table S8. Summary of free energies (G: Hartree) of each stationary points obtained by PBE0-D3/6-31G(d). ${ }^{\text {a }}$

\begin{tabular}{|c|c|c|c|c|}
\hline structure & $G(\mathrm{PBE} 0 / 6-311+\mathrm{G}(\mathrm{d}, \mathrm{p})$ & G (PBE0/def2-TZVP) & G (PBE0-D3/def2-TZVP) & G (PBE0-D3/6-31G(d)) \\
\hline 4 & -2642.249029 & -2642.458558 & -2642.543684 & -2641.897272 \\
\hline $4^{\prime}$ & -2642.249072 & -2642.458597 & -2642.543737 & -2641.897332 \\
\hline $\mathrm{TS}_{4-4^{\prime}}$ & -2642.200632 & -2642.413265 & -2642.487094 & -2641.838408 \\
\hline 7 & -1843.717894 & -1843.922107 & -1843.993645 & -1843.407502 \\
\hline $7^{\prime}$ & -1843.708029 & -1843.912063 & -1843.977606 & -1843.389885 \\
\hline
\end{tabular}

a) $G$ : sum of electronic and thermal correction to free energies $\left(E+\Delta_{G}\right) ; \Delta_{G}$ : thermal correction to free Energy obtained by PBE0-D3/6-31G(d). 


\title{
8. Cartesian coordinates of optimized structures
}

\author{
$(P, P)-1$
}

$(M, M)-1$

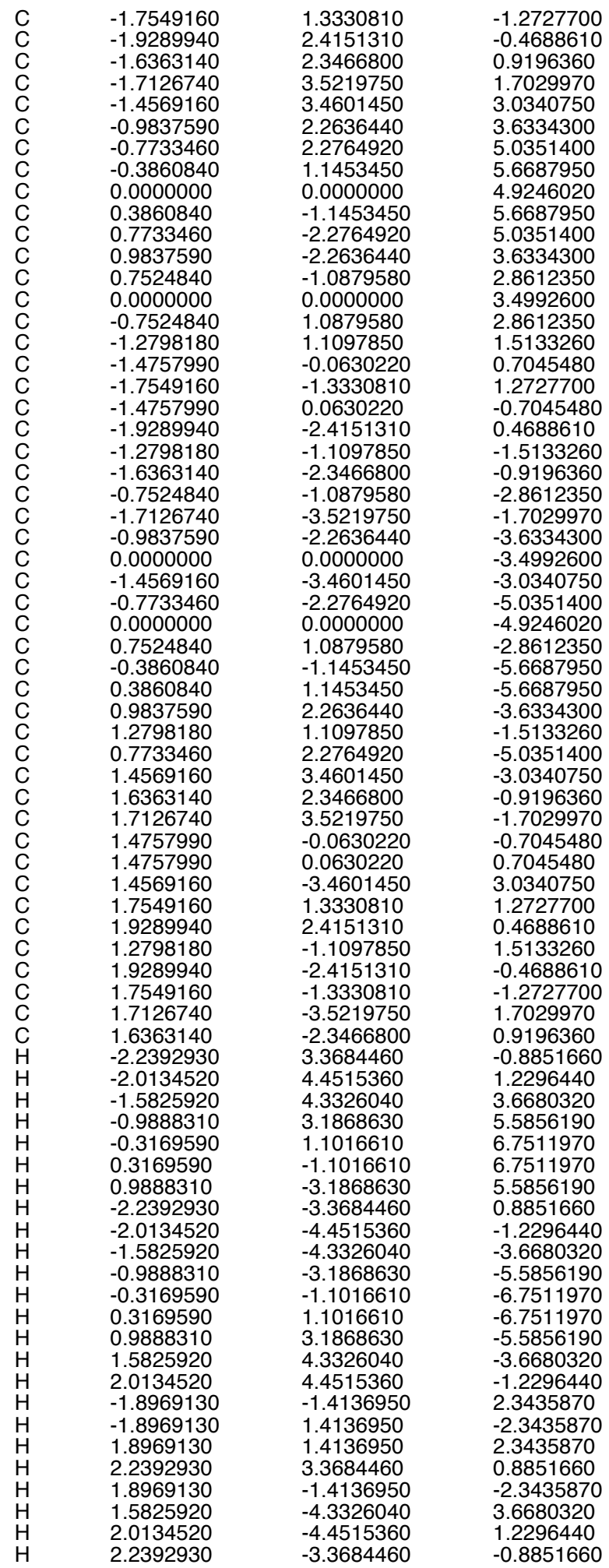

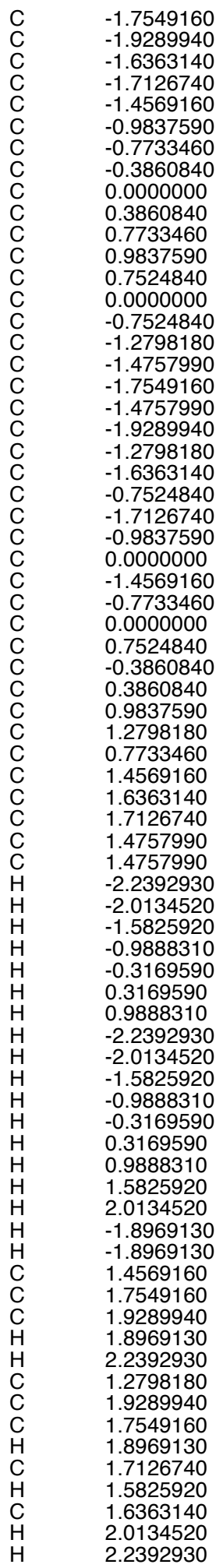

1.3330810 2.4151310 2.3466800 3.5219750 3.4601450 2.2636440 2.2764920

1.1453450 0.0000000

$-1.1453450$

$-2.2764920$

$-2.2636440$

$-1.0879580$

0.0000000

1.0879580

1.1097850

$-0.0630220$

$-1.3330810$

0.0630220

$-2.4151310$

$-1.1097850$

$-2.3466800$

$-1.0879580$

$-3.5219750$

$-2.2636440$

0.0000000

$-3.4601450$

$-2.2764920$

0.0000000

1.0879580

$-1.1453450$

1.1453450

2.2636440

1.1097850

2.2764920

3.4601450

2.3466800

3.5219750

$-0.0630220$

0.0630220

3.3684460

4.4515360

4.3326040

3.1868630

1.1016610

$-1.1016610$

$-3.1868630$

$-3.3684460$

$-4.4515360$

$-4.3326040$

$-3.1868630$

$-1.1016610$

1.1016610

3.1868630

4.3326040

4.4515360

$-1.4136950$

1.4136950

$-3.4601450$

1.3330810

2.4151310

1.4136950

3.3684460

$-1.1097850$

$-2.4151310$

$-1.3330810$

$-1.4136950$

$-3.5219750$

$-4.3326040$

$-2.3466800$

$-4.4515360$

$-3.3684460$
1.2727700

0.4688610

$-0.9196360$

$-1.7029970$

$-3.0340750$

$-3.6334300$

$-5.0351400$

$-5.6687950$

$-4.9246020$

$-5.6687950$

$-5.0351400$

$-3.6334300$

2.8612350

$-3.4992600$

$-2.8612350$

$-1.5133260$

$-0.7045480$

$-1.2727700$

0.7045480

$-0.4688610$

1.5133260

0.9196360

2.8612350

1.7029970

3.6334300

3.4992600

3.0340750

5.0351400

4.9246020

2.8612350

5.6687950

5.6687950

3.6334300

1.5133260

5.0351400

3.0340750

0.9196360

1.7029970

0.7045480

$-0.7045480$

0.8851660

$-1.2296440$

$-3.6680320$

$-5.5856190$

$-6.7511970$

$-6.7511970$

$-5.5856190$

$-0.8851660$

1.2296440

3.6680320

5.5856190

6.7511970

6.7511970

5.5856190

3.6680320

1.2296440

$-2.3435870$

2.3435870

$-3.0340750$

$-1.2727700$

$-0.4688610$

$-2.3435870$

$-0.8851660$

$-1.5133260$

0.4688610

1.2727700

23435870

$-1.7029970$

$-3.6680320$

$-0.9196360$

$-1.2296440$

0.8851660 
$(P, P)-1$ for $\mathrm{NICS}(0)$ and $\mathrm{NICS}(1)$ calculations

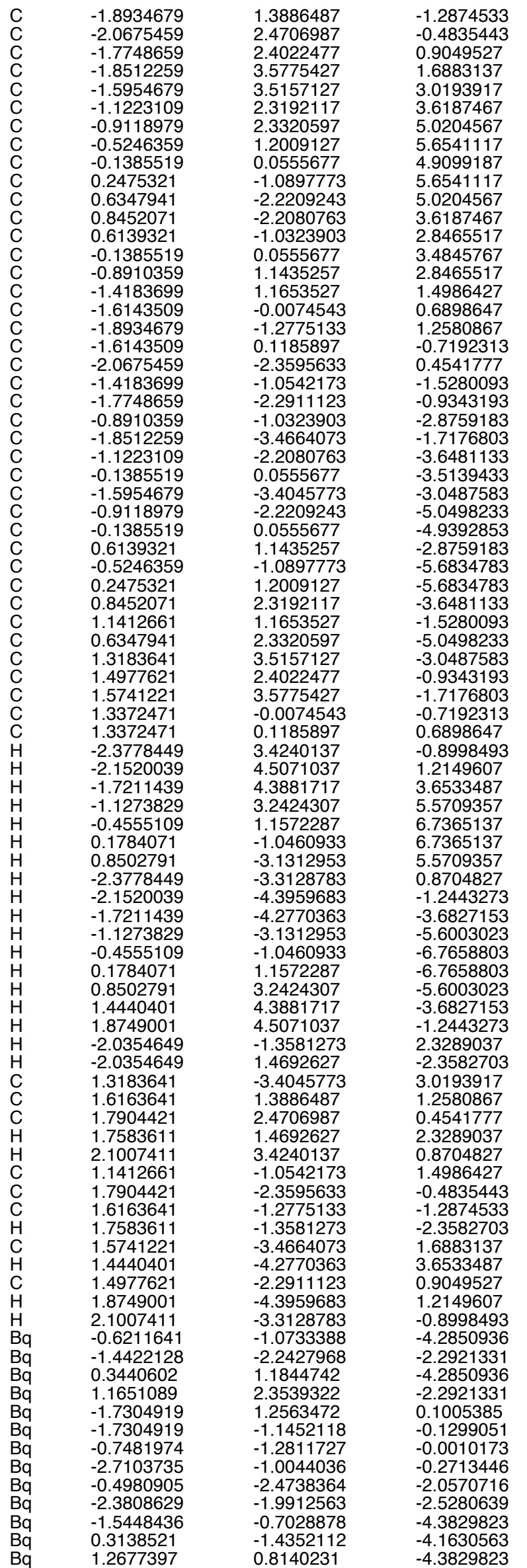

$\begin{array}{llll}\mathrm{Bq} & -0.5816984 & 1.5427637 & -4.1642646 \\ \mathrm{~Bq} & 2.1037590 & 2.1023916 & -2.5280639 \\ \mathrm{~Bq} & 0.2209867 & 2.5849717 & -2.0570716 \\ \mathrm{~Bq} & -0.7481974 & 1.3923080 & -0.0283493 \\ \mathrm{~Bq} & -2.7103735 & 1.1155389 & 0.2419780\end{array}$

\section{benzene}

C $\quad-1.1820870$

$-0.7330860$

0.0000000

0.0000000

0.0000000

0.0000000

0.0000000

0.0000000

0.0000020

$-0.0000010$

$-0.0000010$

0.0000010

$-0.0000010$

$-0.0000010$

\section{phenanthrene}

$\begin{array}{ll}\text { C } & 3.5454770 \\ \text { C } & 2.8251730 \\ \text { C } & 1.4161380 \\ \text { C } & 0.7255560 \\ \text { C } & 1.4911120 \\ \text { C } & 2.8681910 \\ \text { C } & 0.6771420 \\ \text { C } & -0.7255560 \\ \text { C } & -1.4161380 \\ \text { C } & -0.6771420 \\ \text { C } & -2.8251730 \\ H & -3.3359980 \\ \text { C } & -3.5454770 \\ \text { C } & -2.8681910 \\ \text { C } & -1.4911120 \\ H & 1.2292250 \\ H & 4.6301870 \\ H & 3.3359980 \\ H & 0.9970930 \\ H & 3.4303360 \\ H & -1.2292250 \\ H & -4.6301870 \\ H & -3.4303360 \\ H & -0.9970930\end{array}$

-0.2964380
0.8750330
0.8620470
-0.3772390
-1.5599830
-1.5241130
2.0864110
-0.3772390
0.8620470
2.0864110
0.8750330
1.8335980
-0.2964380
-1.5241130
-1.5599830
3.0216340
-0.2728710
1.8335980
-2.5243980
-2.4522790
3.0216340
-0.2728710
-2.4522790
-2.5243980

0.0000000

0.0000000

0.0000000

0.0000000

0.0000000

0.0000000

0.0000000

0.0000000

0.0000000

0.0000000

0.0000000

0.0000000

0.0000000

0.0000000

0.0000000

0.0000000

0.0000000

0.0000000

0.0000000

0.0000000

0.0000000

0.0000000

0.0000000

0.0000000 


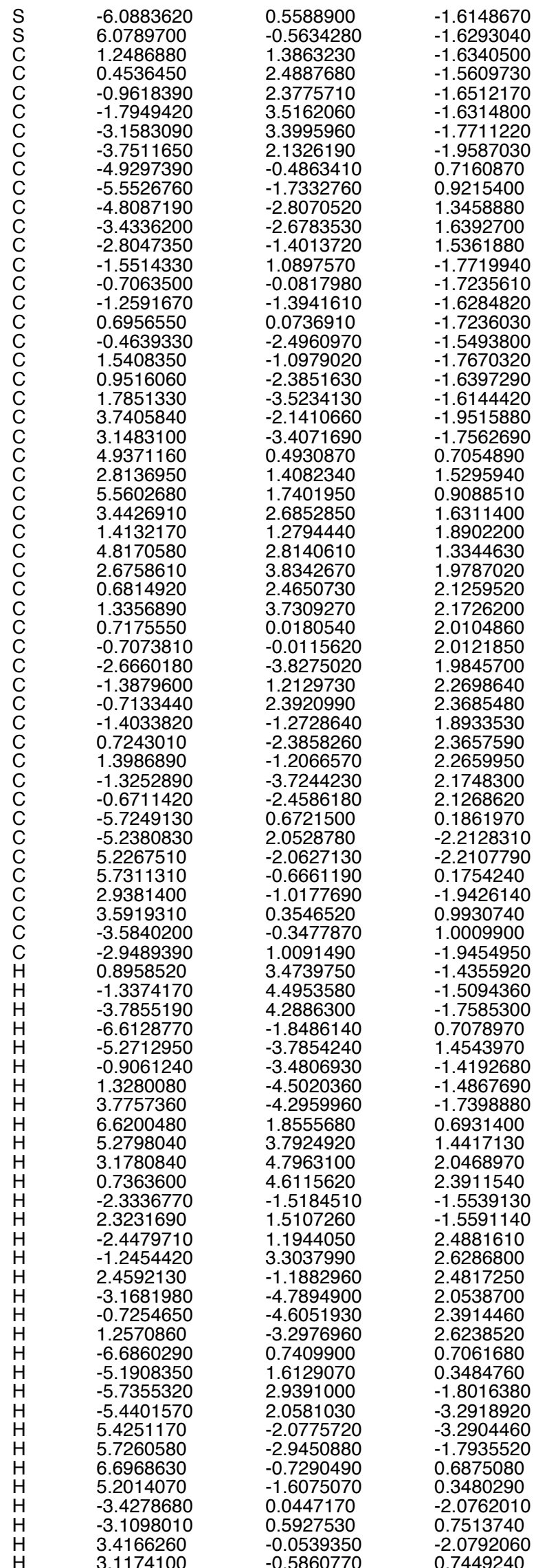

\begin{tabular}{|c|c|c|c|}
\hline S & -6.6127190 & 1.1745450 & 1.6464780 \\
\hline $\mathrm{s}$ & 6.6129130 & 1.1661330 & -1.6528160 \\
\hline C & 0.1523330 & 1.7179130 & -1.8658990 \\
\hline C & -1.1504730 & 1.6808500 & -2.2609410 \\
\hline C & -2.2046310 & 1.8330870 & -1.3188460 \\
\hline C & -3.5542670 & 1.9099790 & -1.7313740 \\
\hline C & -4.5621370 & 2.1196440 & -0.8248960 \\
\hline c & -4.2622670 & 2.2574030 & 0.5476680 \\
\hline c & -4.9973000 & -0.8591070 & 0.5353430 \\
\hline C & -5.8604920 & -1.1814110 & -0.5304280 \\
\hline C & -5.3371370 & -1.6166710 & -1.7218430 \\
\hline c & -3.9477610 & -1.8062660 & -1.8894210 \\
\hline C & -3.0674460 & -1.6027140 & -0.7859570 \\
\hline c & -1.8902580 & 1.9279890 & 0.0622430 \\
\hline $\mathrm{C}$ & -0.5114550 & 1.8325400 & 0.4822010 \\
\hline C & -0.1519490 & 1.7239470 & 1.8579780 \\
\hline C & 0.5118190 & 1.8306680 & -0.4904670 \\
\hline C & 1.1508740 & 1.6871090 & 2.2530270 \\
\hline C & 1.8906860 & 1.9267290 & -0.0708790 \\
\hline C & 2.2051170 & 1.8358820 & 1.3104480 \\
\hline C & 3.5548540 & 1.9134050 & 1.7226240 \\
\hline C & 4.2627510 & 2.2536230 & -0.5575640 \\
\hline C & 4.5626920 & 2.1200470 & 0.8153930 \\
\hline C & 4.9971770 & -0.8616780 & -0.5319390 \\
\hline C & 3.0671240 & -1.5981740 & 0.7931030 \\
\hline C & 5.8602260 & -1.1781980 & 0.5356630 \\
\hline C & 3.9472640 & -1.7952090 & 1.8978950 \\
\hline C & 1.6506830 & -1.8732560 & 0.9599730 \\
\hline C & 5.3366870 & -1.6068400 & 1.7293870 \\
\hline C & 3.4226910 & -2.1216320 & 3.1808080 \\
\hline C & 1.1772170 & -2.1030800 & 2.2720220 \\
\hline C & 2.0813740 & -2.2174960 & 3.3685530 \\
\hline C & 0.7009670 & -1.9576080 & -0.1284020 \\
\hline C & -0.7012250 & -1.9568860 & 0.1374070 \\
\hline C & -3.4233300 & -2.1405030 & -3.1703960 \\
\hline $\mathrm{C}$ & -1.1127500 & -2.1951820 & 1.4802980 \\
\hline C & -0.2084060 & -2.3003700 & 2.4933040 \\
\hline C & -1.6510530 & -1.8788980 & -0.9513410 \\
\hline C & 0.2079610 & -2.3148400 & -2.4822400 \\
\hline C & 1.1124060 & -2.2033940 & -1.4699680 \\
\hline C & -2.0820370 & -2.2376320 & -3.3577230 \\
\hline C & -1.1777270 & -2.1166190 & -2.2620240 \\
\hline C & -5.5522440 & -0.3038270 & 1.8200050 \\
\hline C & -5.3532920 & 2.4963890 & 1.5502100 \\
\hline C & 5.3538210 & 2.4886610 & -1.5610480 \\
\hline C & 5.5520930 & -0.3127880 & -1.8193280 \\
\hline C & 2.9526200 & 2.1451610 & -0.9771420 \\
\hline C & 3.6388410 & -1.0608960 & -0.3851470 \\
\hline C & -3.6389460 & -1.0586420 & 0.3892700 \\
\hline C & -2.9522190 & 2.1496740 & 0.9677110 \\
\hline $\mathrm{H}$ & -1.4035930 & 1.5541380 & -3.3105270 \\
\hline $\mathrm{H}$ & -3.7811740 & 1.8046840 & -2.7894790 \\
\hline $\mathrm{H}$ & -5.5983800 & 2.1616150 & -1.1491940 \\
\hline $\mathrm{H}$ & -6.9302160 & -1.0225910 & -0.4219880 \\
\hline $\mathrm{H}$ & -5.9905440 & -1.8058390 & -2.5706430 \\
\hline $\mathrm{H}$ & 1.4039400 & 1.5633600 & 3.3029830 \\
\hline $\mathrm{H}$ & 3.7818970 & 1.8108490 & 2.7809680 \\
\hline $\mathrm{H}$ & 5.5990350 & 2.1624360 & 1.1392960 \\
\hline $\mathrm{H}$ & 6.9299940 & -1.0201530 & 0.4265490 \\
\hline $\mathrm{H}$ & 5.9899860 & -1.7913700 & 2.5792910 \\
\hline $\mathrm{H}$ & 4.1143280 & -2.2548790 & 4.0093230 \\
\hline $\mathrm{H}$ & 1.6708700 & -2.4224980 & 4.3544430 \\
\hline $\mathrm{H}$ & -0.9305100 & 1.6234410 & 2.6065450 \\
\hline $\mathrm{H}$ & 0.9308290 & 1.6145750 & -2.6141470 \\
\hline $\mathrm{H}$ & -2.1543740 & -2.4031640 & 1.6866270 \\
\hline $\mathrm{H}$ & -0.5432340 & -2.5548960 & 3.4961750 \\
\hline $\mathrm{H}$ & 2.1540490 & -2.4122710 & -1.6752490 \\
\hline $\mathrm{H}$ & -4.1150550 & -2.2786560 & -3.9980330 \\
\hline $\mathrm{H}$ & -1.6716740 & -2.4485780 & -4.3424170 \\
\hline $\mathrm{H}$ & 0.5427880 & -2.5751090 & -3.4836370 \\
\hline $\mathrm{H}$ & -6.2036110 & -1.0433600 & 2.3011250 \\
\hline $\mathrm{H}$ & -4.7472240 & -0.0803530 & 2.5289490 \\
\hline $\mathrm{H}$ & -4.9262310 & 2.6531310 & 2.5468040 \\
\hline $\mathrm{H}$ & -5.9322350 & 3.3930790 & 1.2987060 \\
\hline $\mathrm{H}$ & 5.9330570 & 3.3860790 & -1.3128230 \\
\hline $\mathrm{H}$ & 4.9266820 & 2.6419530 & -2.5581460 \\
\hline $\mathrm{H}$ & 6.2032850 & -1.0546990 & -2.2970210 \\
\hline $\mathrm{H}$ & 4.7470270 & -0.0924730 & -2.5292100 \\
\hline $\mathrm{H}$ & -2.7395880 & 2.2697560 & 2.0262480 \\
\hline $\mathrm{H}$ & -2.9878570 & -0.7146950 & 1.1830110 \\
\hline $\mathrm{H}$ & 2.7398840 & 2.2619900 & -2.0360220 \\
\hline 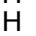 & 2.9879190 & -0.7216370 & -1.1810310 \\
\hline
\end{tabular}




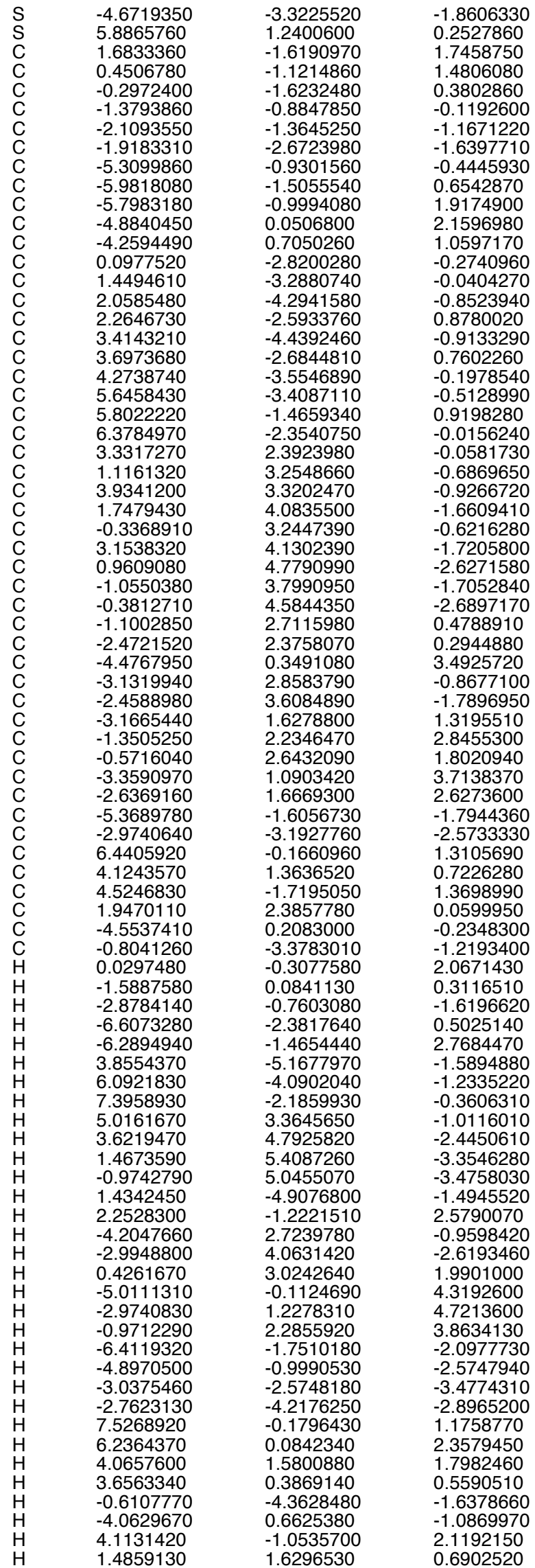

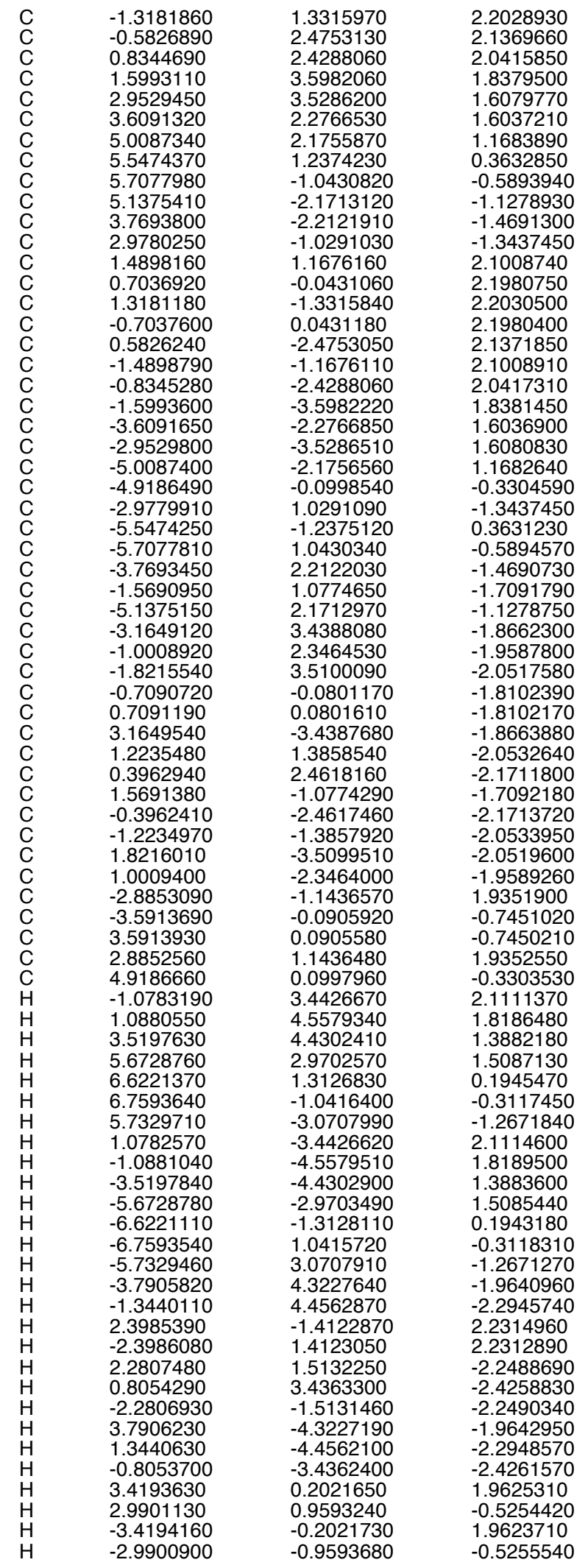




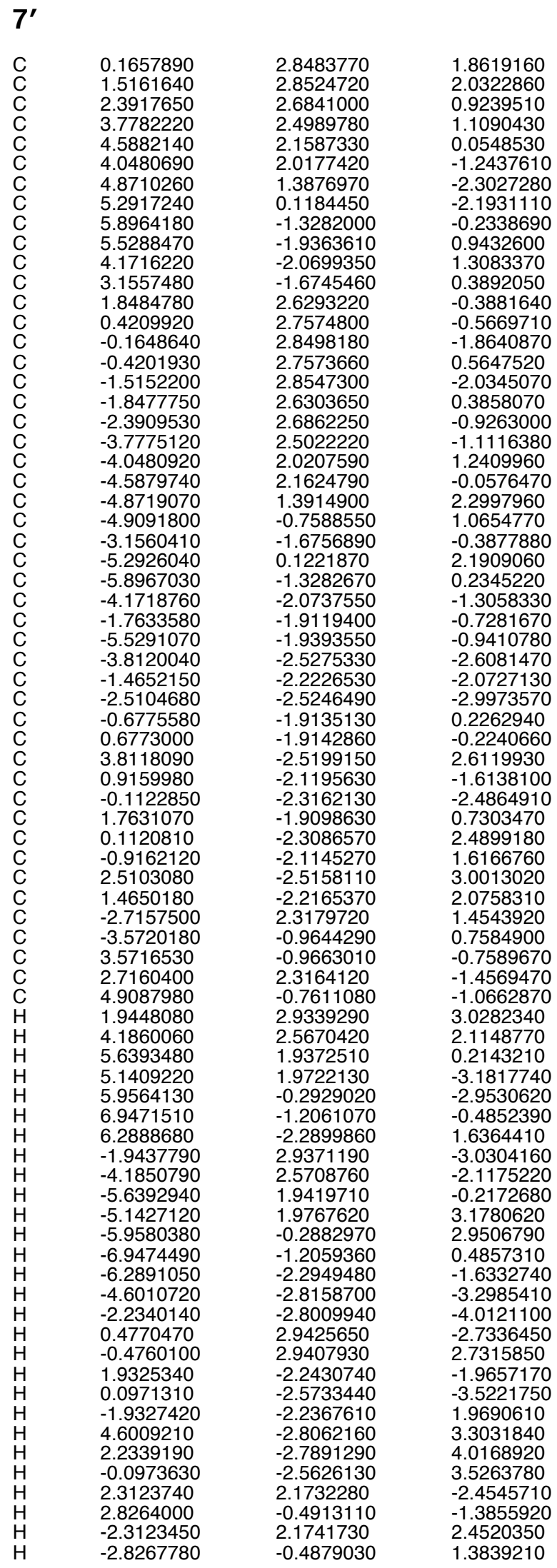




\section{References}

(1) Jouanno, C.; Le Floc'h, Y.; Gree, R. A CONVENIENT ACCES TO 1,7-DIFUNCTIONAL NAPHTHALENES: THE SYNTHESIS OF THE 1,7-DICARBALDEHYDE 7-MONODIMETHYLACETAL. Bull. Soc. Chim. Belg. 1995, 104, 49-53.

(2) Zrida, H.; Hriz, K.; Jaballah, N.; Kreher, D.; Majdoub, M. Synthesis and study of morphological, optical and electrical properties of new organic semi conducting polymers containing isosorbide pendant group. Synth. Met. 2016, 221, 227-235.

(3) (a) Sudhakar, A.; Katz, T. J. Directive effect of bromine on stilbene photocyclizations. an improved synthesis of [7]helicene. Tetrahedron Lett. 1986, 27, 2231-2234. (b) Liu, L.; Katz, T. J. Bromine auxiliaries in photosyntheses of [5]helicenes. Tetrahedron Lett. 1991, 32, 6831-6834.

(4) Sheldrick, G. SHELXT - Integrated space-group and crystal-structure determination. Acta Crystallogr. A 2015, A71, 3-8.

(5) Sheldrick, G. Crystal structure refinement with SHELXL. Acta Crystallogr. 2015, C71, 3-8.

(6) Dolomanov, O. V.; Bourhis, L. J.; Gildea, R. J.; Howard, J. A. K.; Puschmann, H. OLEX2: a complete structure solution, refinement and analysis program. J. Appl. Crystallogr. 2009, 42, 339-341.

(7) Frisch, M. J.; Trucks, G. W.; Schlegel, H. B.; Scuseria, G. E.; Robb, M. A.; Cheeseman, J. R.; Scalmani, G.; Barone, V.; Petersson, G. A.; Nakatsuji, H.; Li, X.; Caricato, M.; Marenich, A. V.; Bloino, J.; Janesko, B. G.; Gomperts, R.; Mennucci, B.; Hratchian, H. P.; Ortiz, J. V.; Izmaylov, A. F.; Sonnenberg, J. L.; Williams; Ding, F.; Lipparini, F.; Egidi, F.; Goings, J.; Peng, B.; Petrone, A.; Henderson, T.; Ranasinghe, D.; Zakrzewski, V. G.; Gao, J.; Rega, N.; Zheng, G.; Liang, W.; Hada, M.; Ehara, M.; Toyota, K.; Fukuda, R.; Hasegawa, J.; Ishida, M.; Nakajima, T.; Honda, Y.; Kitao, O.; Nakai, H.; Vreven, T.; Throssell, K.; Montgomery Jr., J. A.; Peralta, J. E.; Ogliaro, F.; Bearpark, M. J.; Heyd, J. J.; Brothers, E. N.; Kudin, K. N.; Staroverov, V. N.; Keith, T. A.; Kobayashi, R.; Normand, J.; Raghavachari, K.; Rendell, A. P.; Burant, J. C.; Iyengar, S. S.; Tomasi, J.; Cossi, M.; Millam, J. M.; Klene, M.; Adamo, C.; Cammi, R.; Ochterski, J. W.; Martin, R. L.; Morokuma, K.; Farkas, O.; Foresman, J. B.; Fox, D. J. Gaussian 16 Rev. C.01, Wallingford, CT, 2016.

(8) Adamo, C.; Barone, V. Toward reliable density functional methods without adjustable parameters: The PBE0 model, J. Chem. Phys. 1999, 110, 6158-6169.

(9) (a) Becke, A. D. Density-functional thermochemistry. III. The role of exact exchange. J. Chem. Phys. 1993, 98, 5648-5652. (b) Lee, C.; Yang, W.; Parr, R. G. Development of the Colle-Salvetti correlationenergy formula into a functional of the electron density. Phys. Rev. B 1988, 37, 785-789.

(10) (a) London, L. The quantic theory of inter-atomic currents in aromatic combinations, J. Phys. Radium, 1937, 8, 397-409. (b) McWeeny, R. Perturbation Theory for Fock-Dirac Density Matrix. Phys. Rev. 1962, 126, 1028. (c) Ditchfield, R. Self-consistent perturbation theory of diamagnetism. 1. Gaugeinvariant LCAO method for N.M.R. chemical shifts. Mol. Phys. 1974, 27, 789-807. (d) Cheeseman, J. R.; Trucks, G. W.; Keith, T. A.; Frisch, M. J. A Comparison of Models for Calculating Nuclear Magnetic Resonance Shielding Tensors. J. Chem. Phys. 1996, 104, 5497-509. (e) Ebrahimi, H. P.; Shaghaghi, $\mathrm{H}$;; Tafazzoli, M. Gauge invariant atomic orbital-density functional theory prediction of accurate gas phase ${ }^{1} \mathrm{H}$ and ${ }^{13} \mathrm{C}$ NMR chemical shifts. Concepts Magn. Reson. 2011, 38A, 269-279.

(11) (a) Schleyer, P. v. R.; Maerker, C.; Dransfeld, A.; Jiao, H.; van Eikema Hommes, N. J. R. NucleusIndependent Chemical Shifts: A Simple and Efficient Aromatocity Probe. J. Am. Chem. Soc. 1996, 118, 6317-6318. (b) Chen, Z.; Wannere, C. S.; Corminboeuf, C.; Puchta, R.; Schleyer, P. V. R. Nucleus-independent chemical shifts (NICS) as an aromaticity criterion. Chem. Rev. 2005, 105, 38423888.

(12) Marenich, A. V.; Cramer, C. J.; Truhlar, D. J. Universal Solvation Model Based on Solute Electron Density and on a Continuum Model of the Solvent Defined by the Bulk Dielectric Constant and Atomic Surface Tensions. J. Phys. Chem. B 2009, 113, 6378-6396.

(13) Dennington, R.; Keith, T. A.; Millam, J. M. GaussView, Version 6, Semichem Inc., Shawnee Mission, KS, 2016. 
(a)

10. ${ }^{1} \mathrm{H}$ and ${ }^{13} \mathrm{C}$ NMR spectra of synthesized compounds

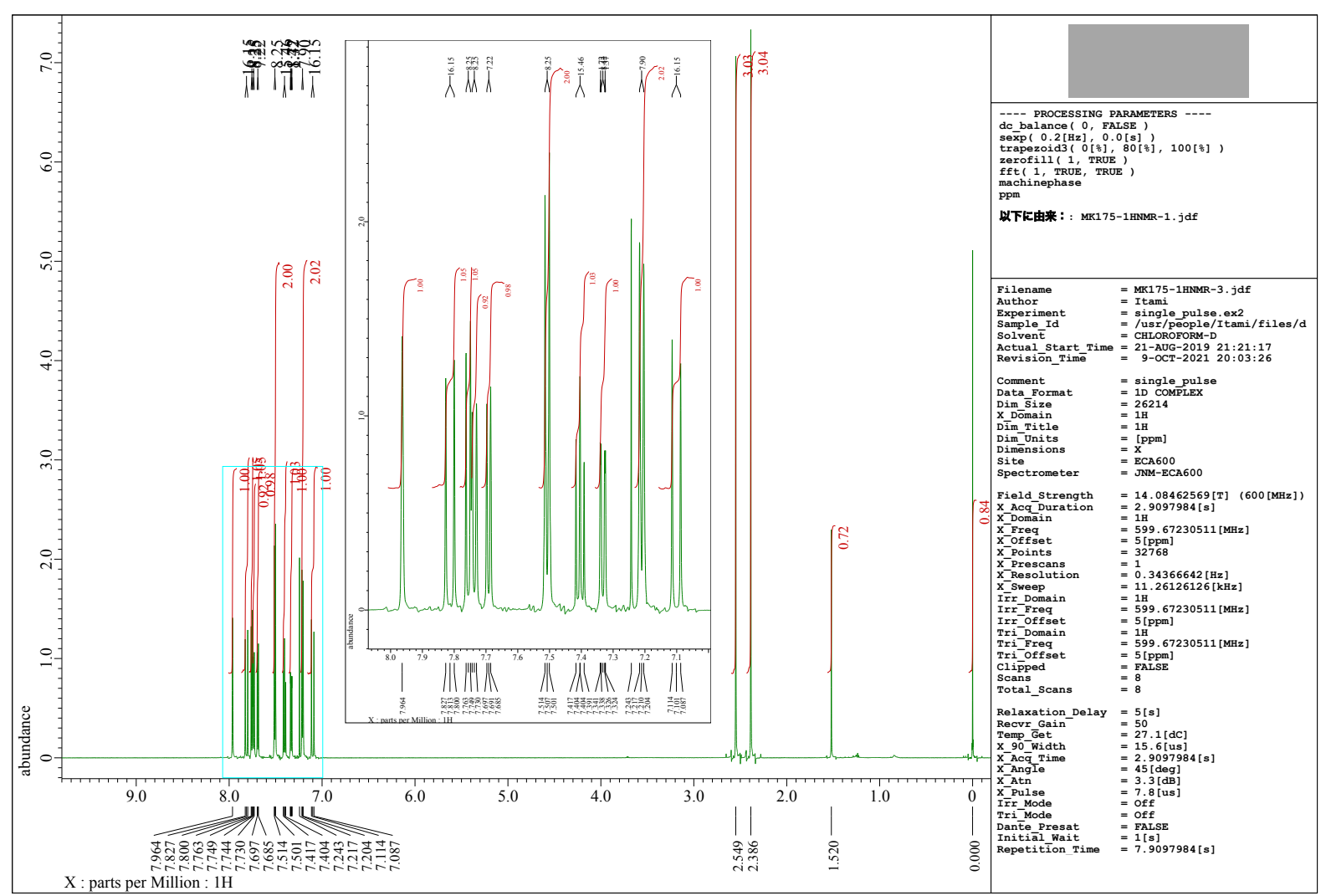

(b)

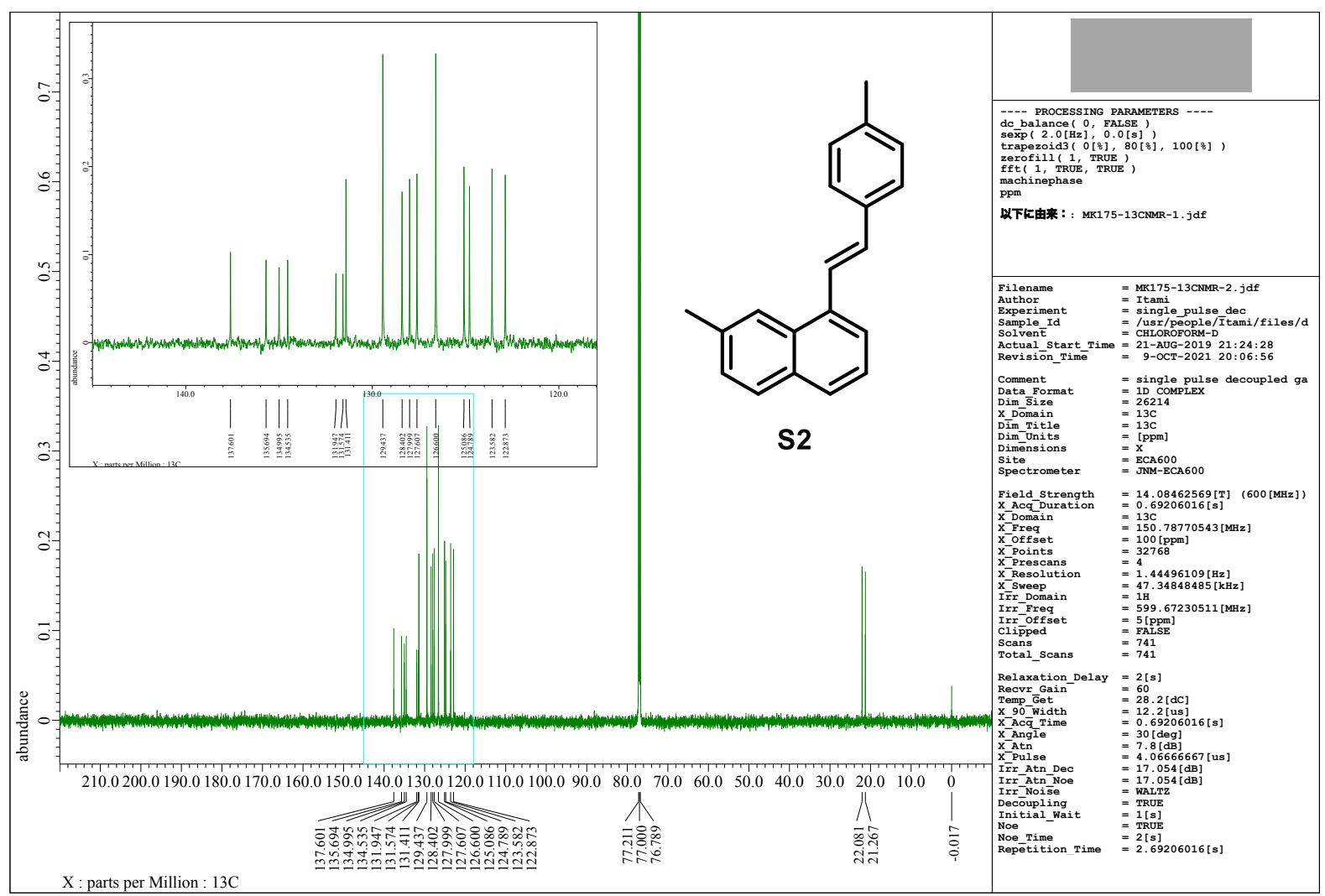

Figure S16. (a) ${ }^{1} \mathrm{H}$ NMR of $\mathbf{S 2}\left(600 \mathrm{MHz}, \mathrm{CDCl}_{3}\right) ;(\mathrm{b}){ }^{13} \mathrm{C}$ NMR of $\mathbf{S} 2\left(151 \mathrm{MHz}, \mathrm{CDCl}_{3}\right)$ 
(a)

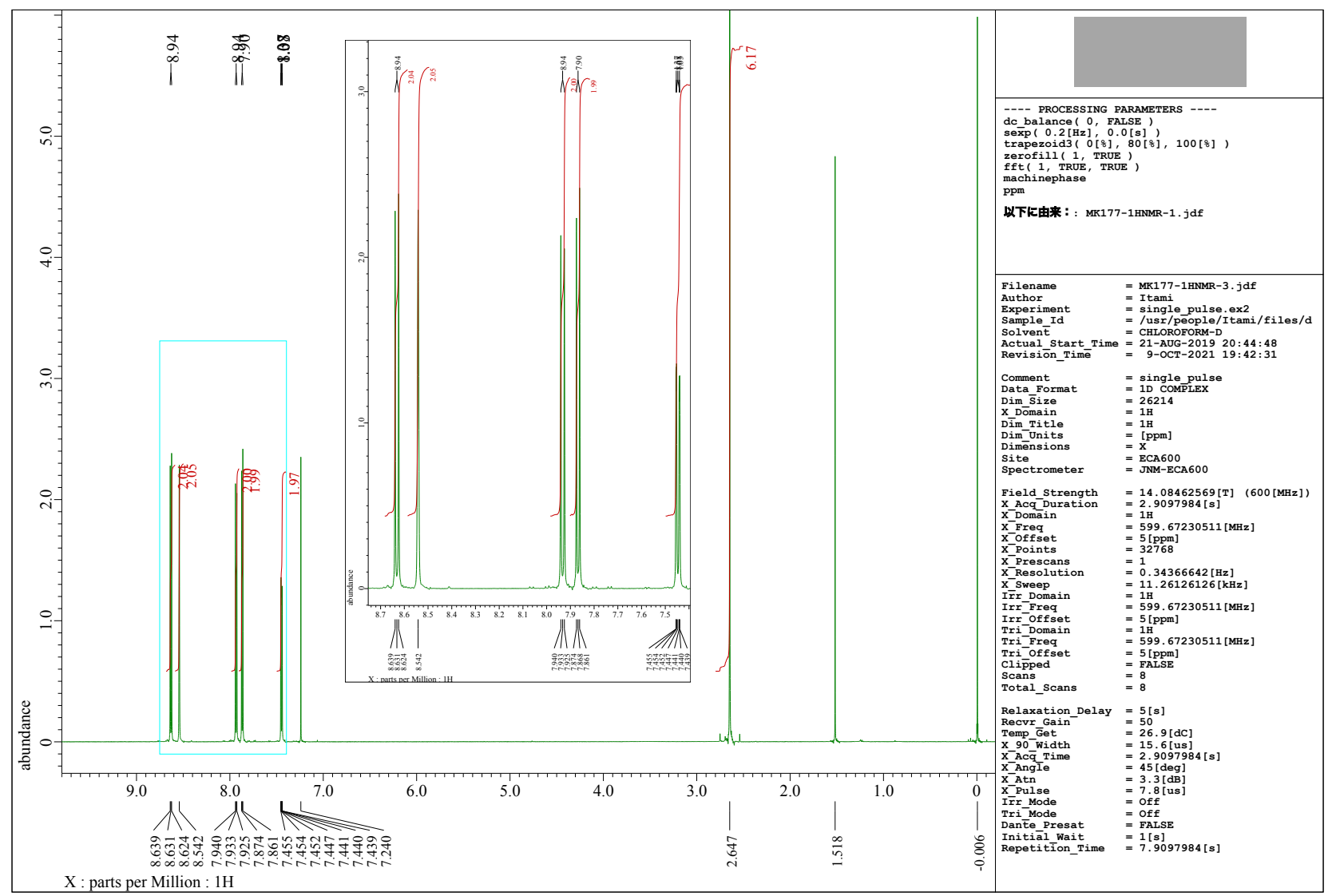

(b)

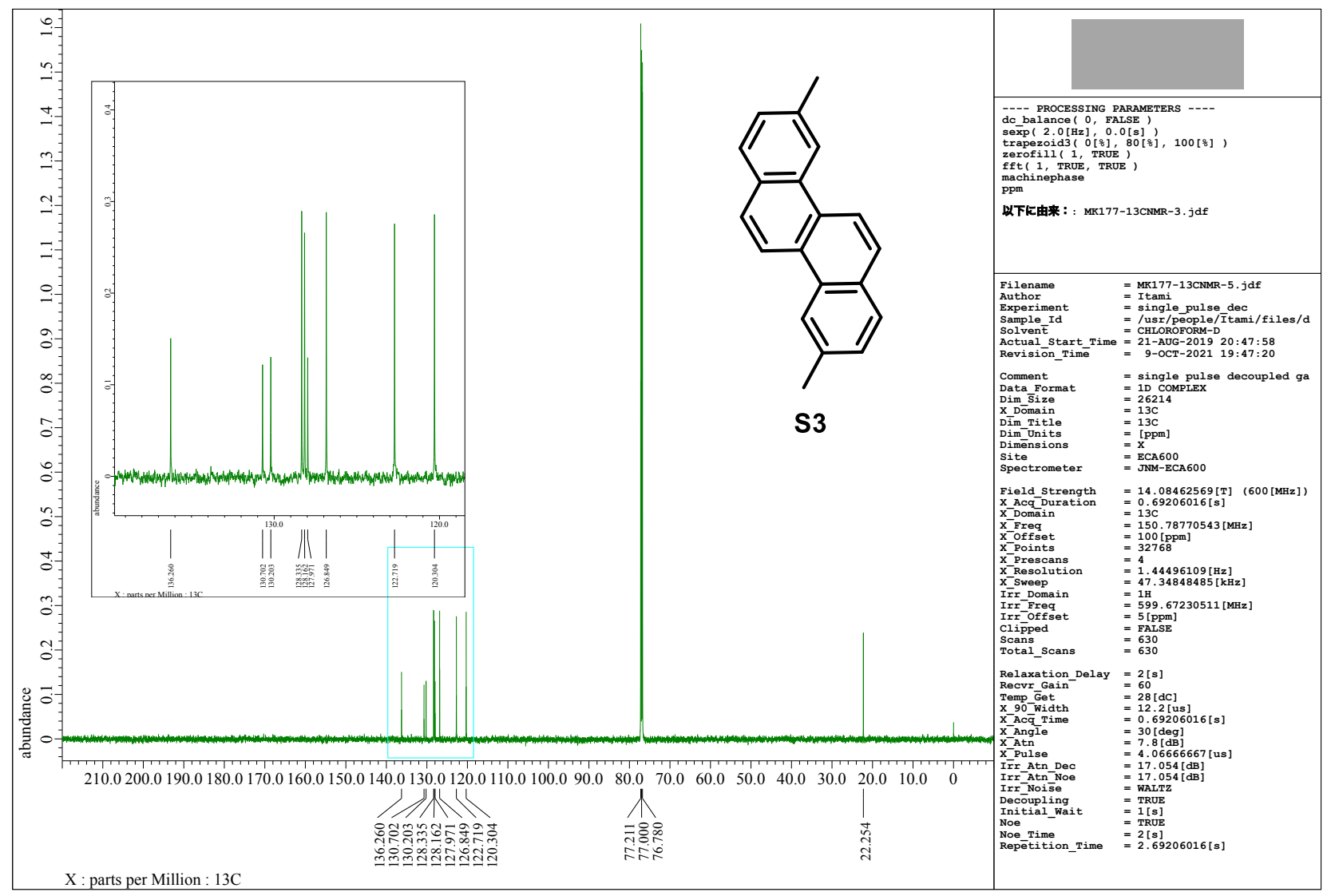

Figure S17. (a) ${ }^{1} \mathrm{H}$ NMR of $\mathbf{S} 3\left(600 \mathrm{MHz}, \mathrm{CDCl}_{3}\right)$; (b) ${ }^{13} \mathrm{C} \mathrm{NMR}$ of $\mathbf{S 3}\left(151 \mathrm{MHz}, \mathrm{CDCl}_{3}\right)$ 
(a)

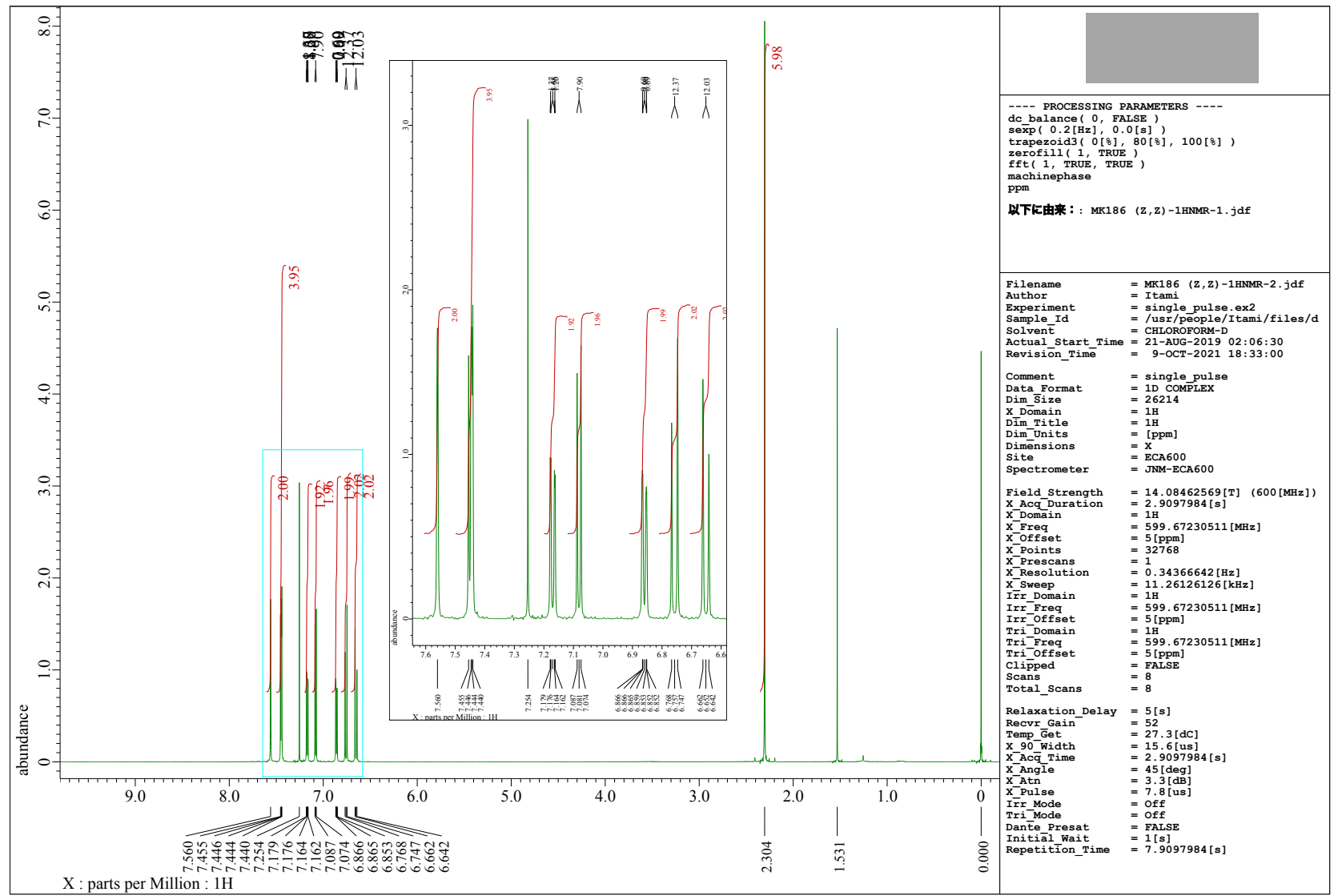

(b)

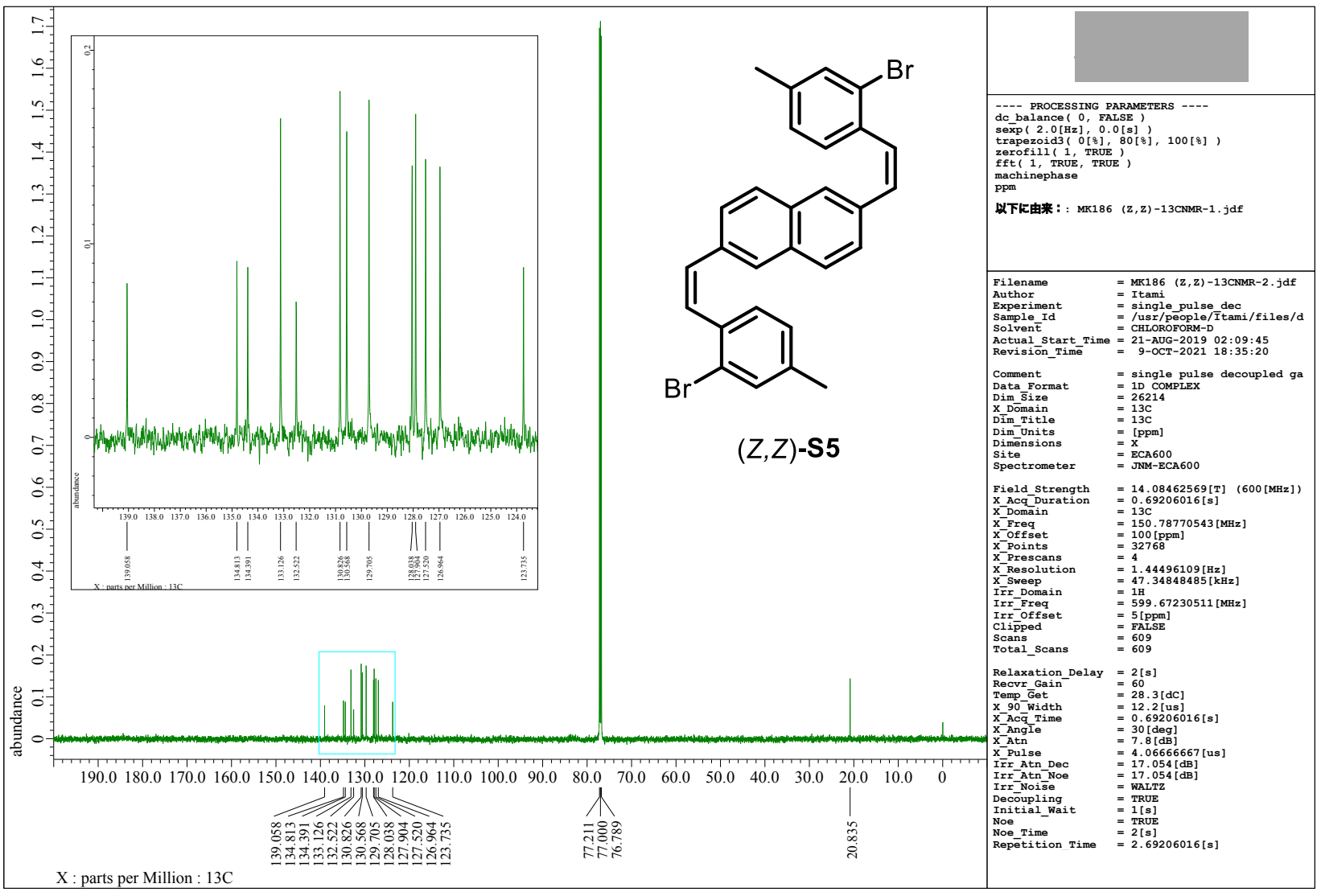

Figure S18. (a) ${ }^{1} \mathrm{H}$ NMR of $(Z, Z)-S 5\left(600 \mathrm{MHz}, \mathrm{CDCl}_{3}\right) ;(\mathrm{b}){ }^{13} \mathrm{C}$ NMR of $(Z, Z)-\mathrm{S} 5\left(151 \mathrm{MHz}, \mathrm{CDCl}_{3}\right)$ 
(a)

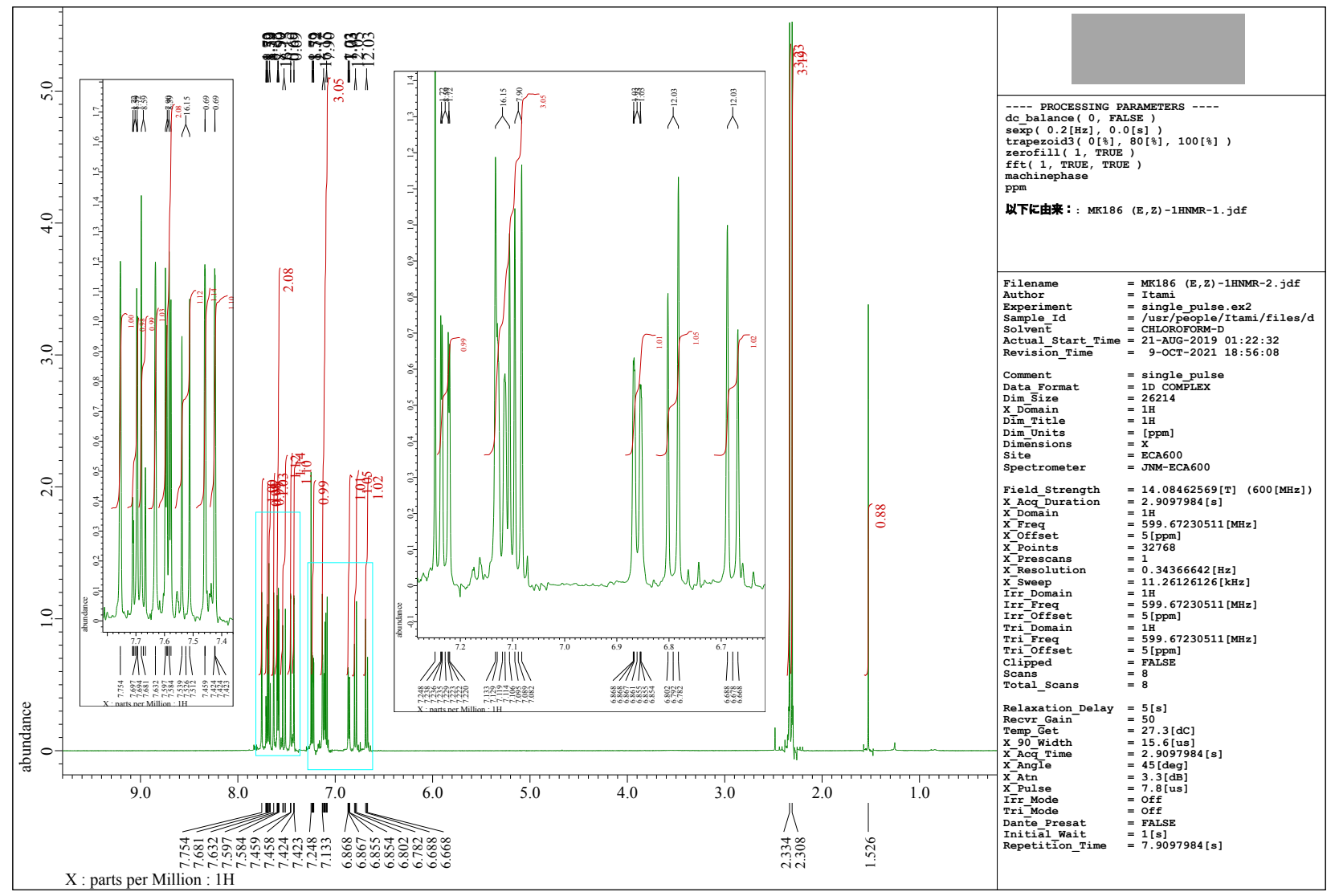

(b)

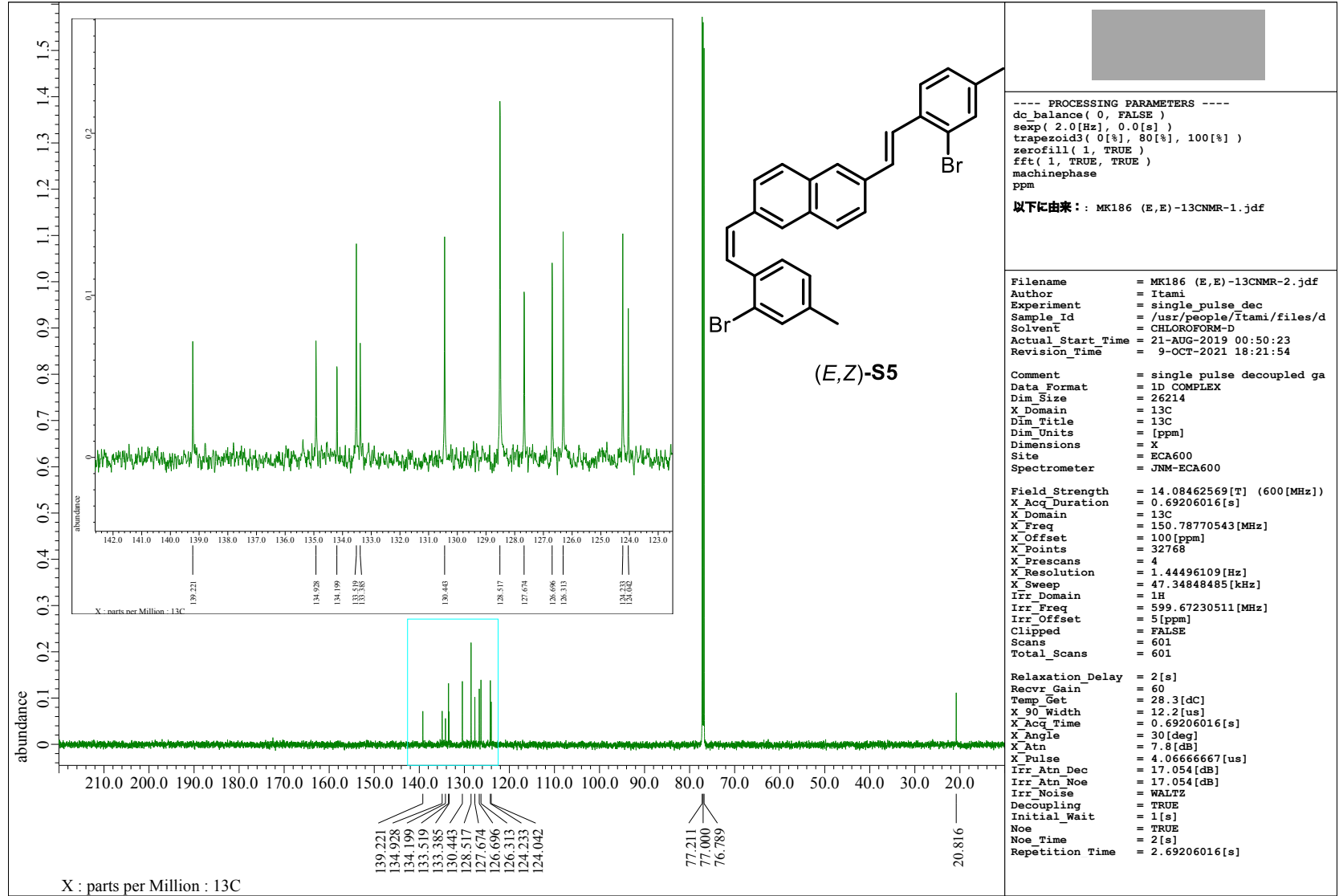

Figure S19. (a) ${ }^{1} \mathrm{H}$ NMR of $(E, Z)-S 5\left(600 \mathrm{MHz}, \mathrm{CDCl}_{3}\right) ;(\mathrm{b}){ }^{13} \mathrm{C}$ NMR of $(E, Z)-\mathbf{S} 5\left(151 \mathrm{MHz}, \mathrm{CDCl}_{3}\right)$ 
(a)

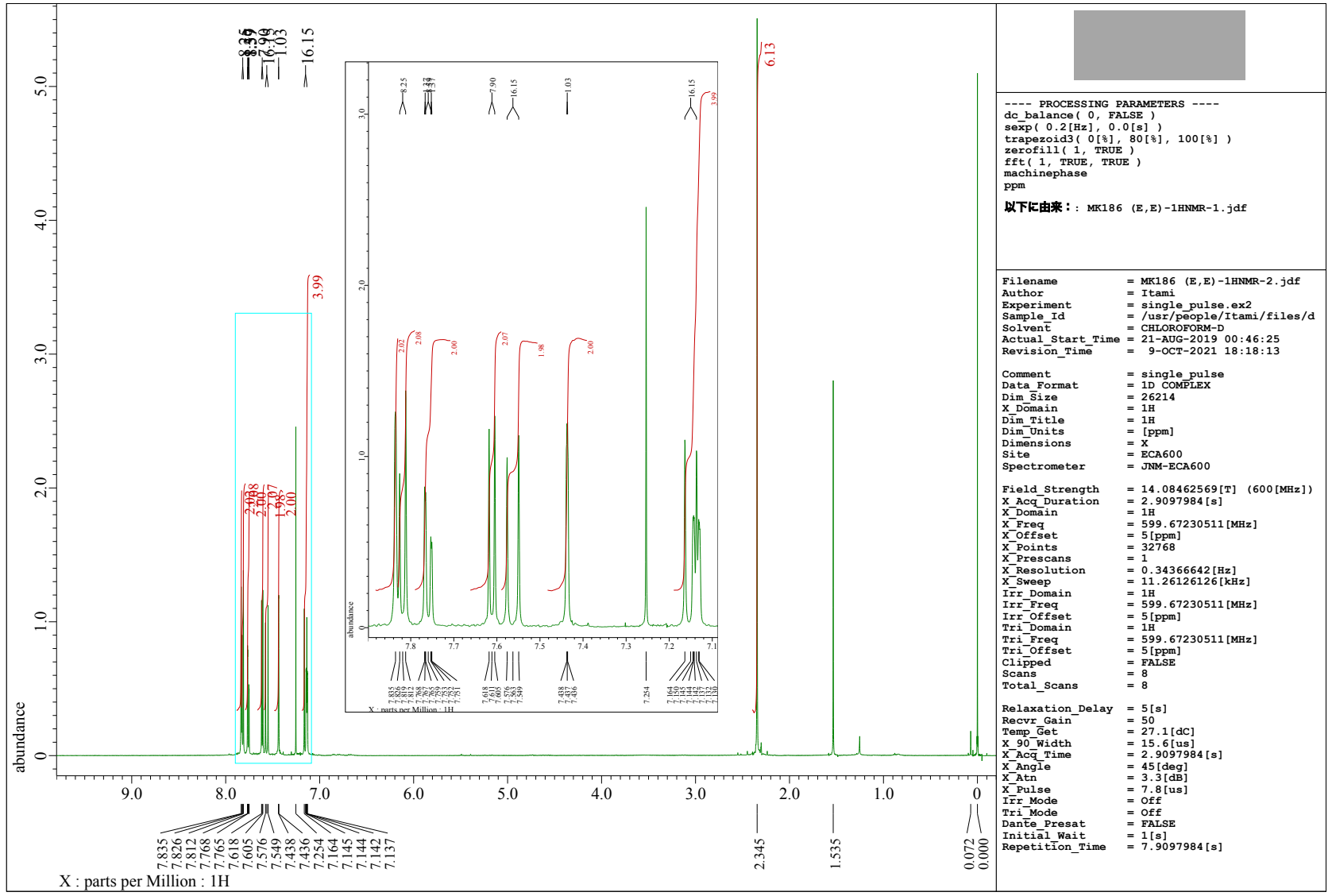

(b)

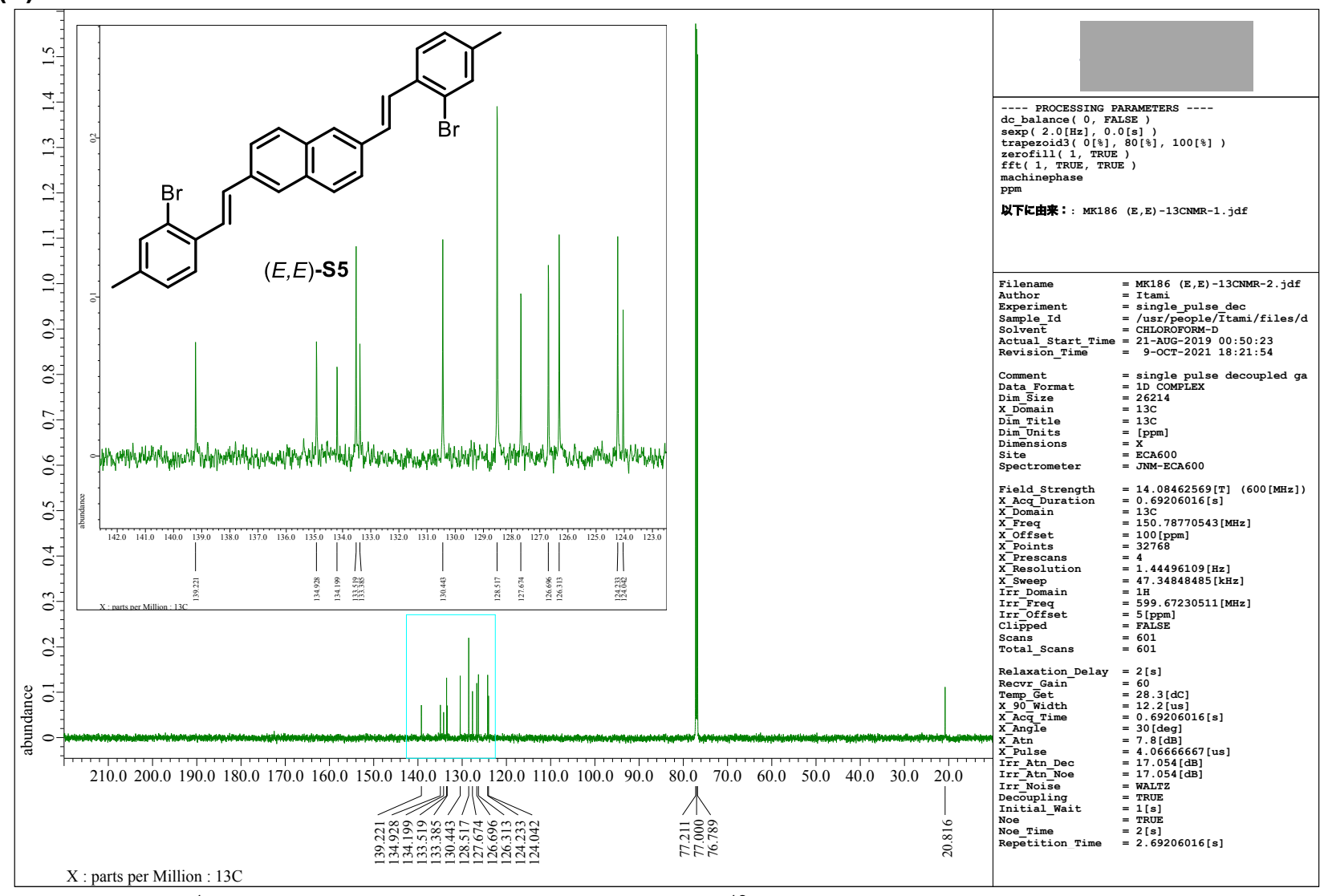

Figure S20. (a) ${ }^{1} \mathrm{H}$ NMR of $(E, E)-\mathbf{S} 5\left(600 \mathrm{MHz}, \mathrm{CDCl}_{3}\right) ;(\mathrm{b}){ }^{13} \mathrm{C}$ NMR of $(E, E)-\mathbf{S 5}\left(151 \mathrm{MHz}, \mathrm{CDCl}_{3}\right)$ 
(a)

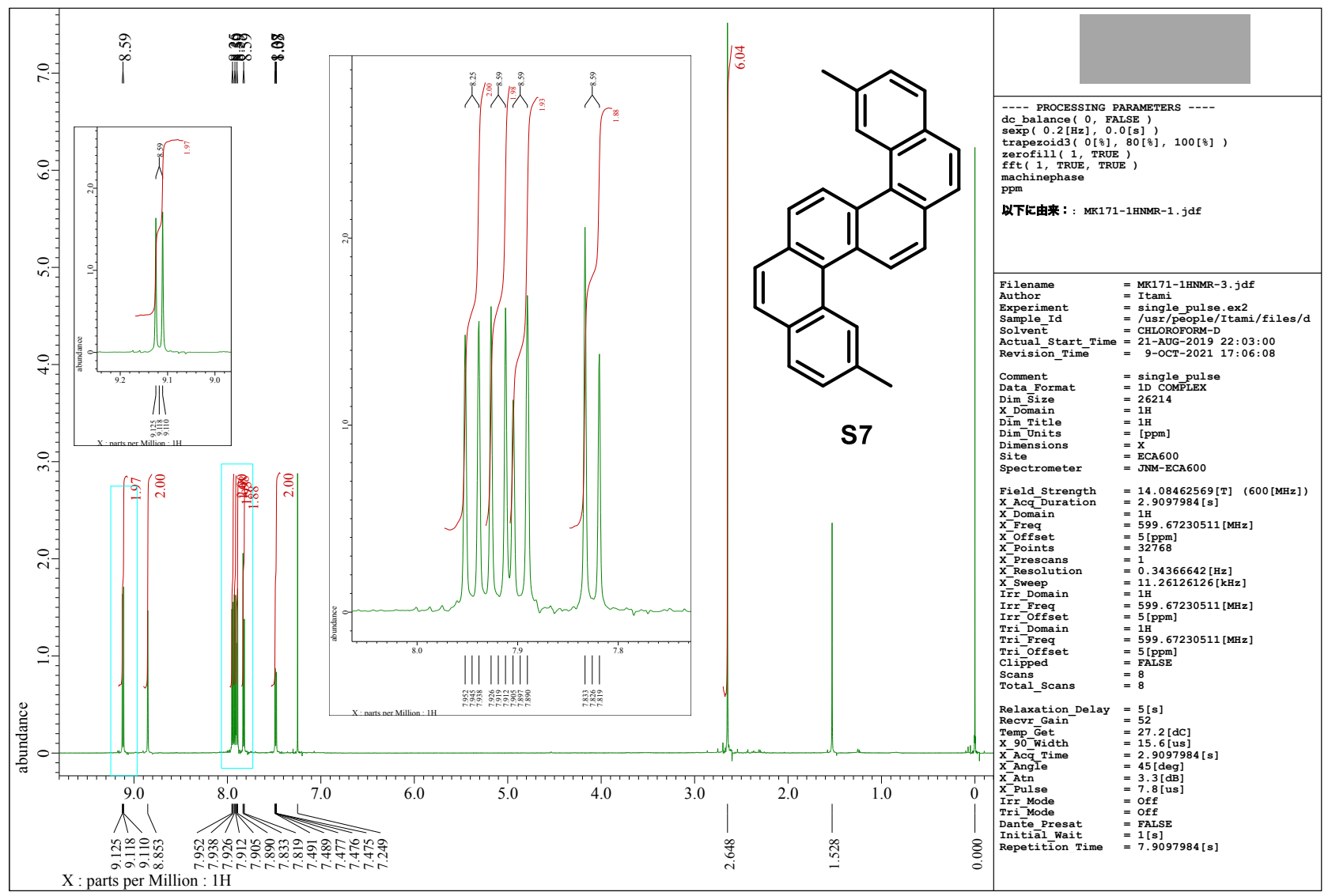

(b)

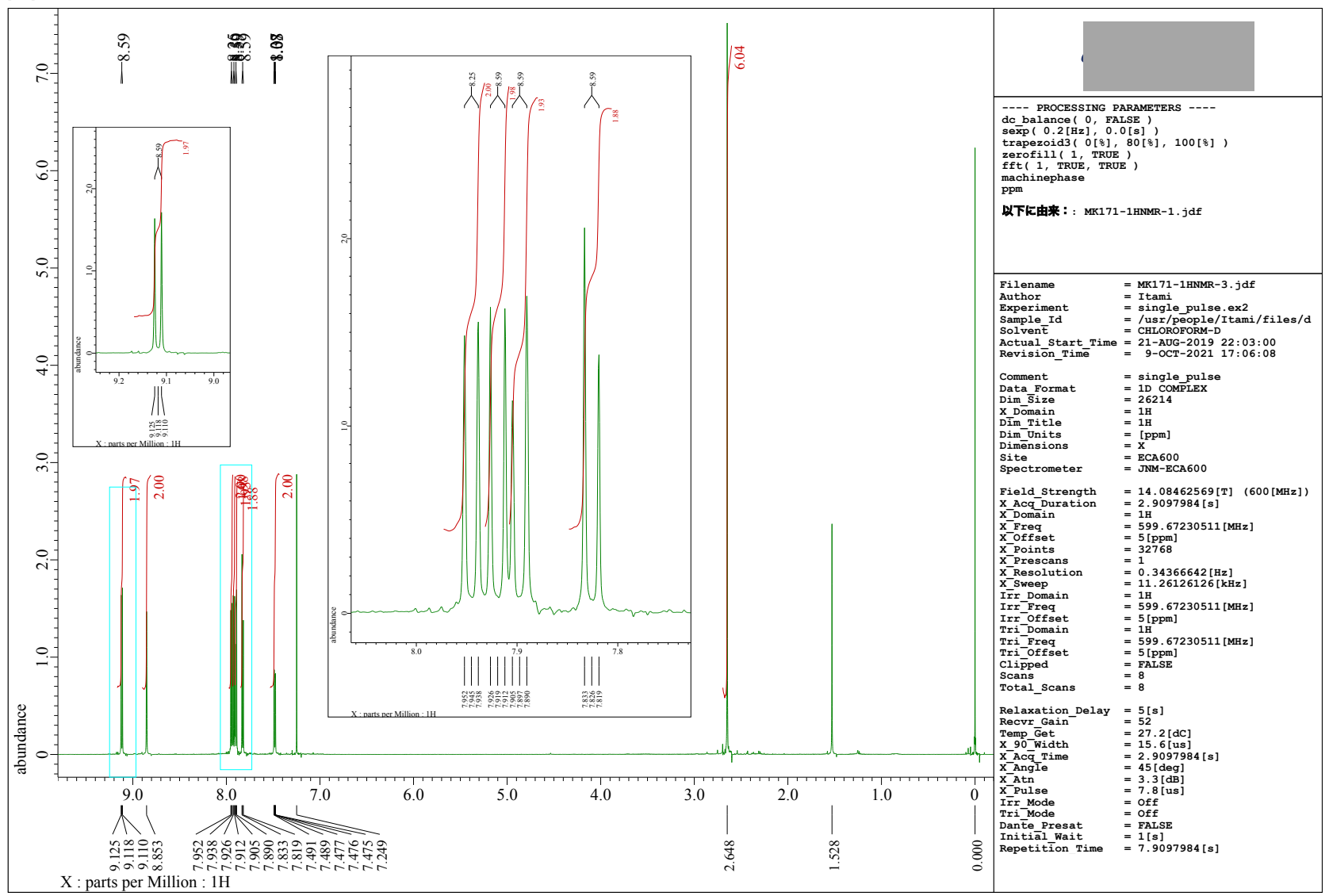

Figure S21. (a) ${ }^{1} \mathrm{H}$ NMR of $\mathbf{S 7}\left(600 \mathrm{MHz}, \mathrm{CDCl}_{3}\right) ;(\mathrm{b}){ }^{13} \mathrm{C}$ NMR of $\mathbf{S 7}\left(151 \mathrm{MHz}, \mathrm{CDCl}_{3}\right)$ 
(a)

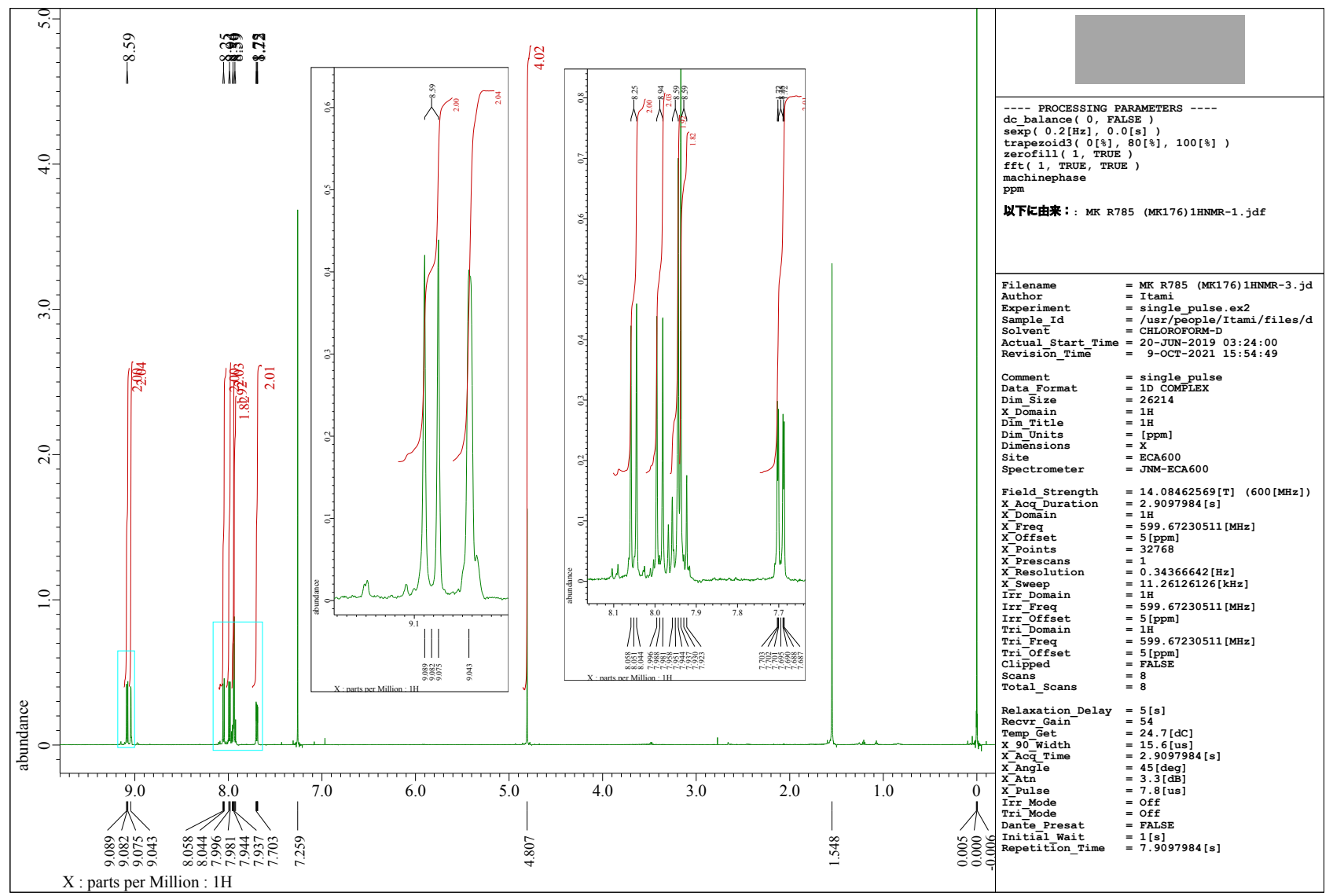

(b)

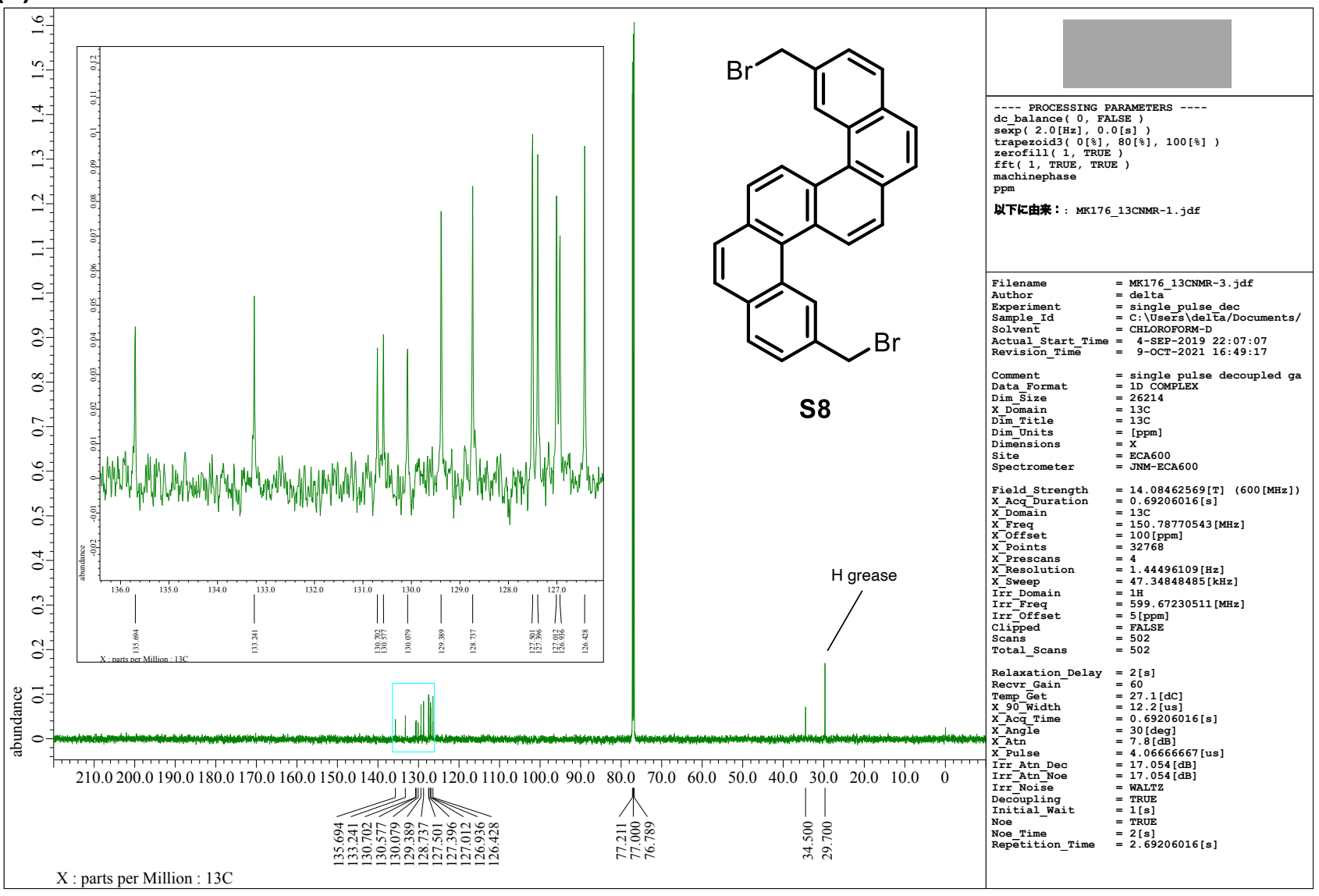

Figure S22. (a) ${ }^{1} \mathrm{H}$ NMR of $\mathbf{S 8}\left(600 \mathrm{MHz}, \mathrm{CDCl}_{3}\right)$; (b) ${ }^{13} \mathrm{C}$ NMR of $\mathbf{S 8}\left(151 \mathrm{MHz}, \mathrm{CDCl}_{3}\right)$ 
(a)

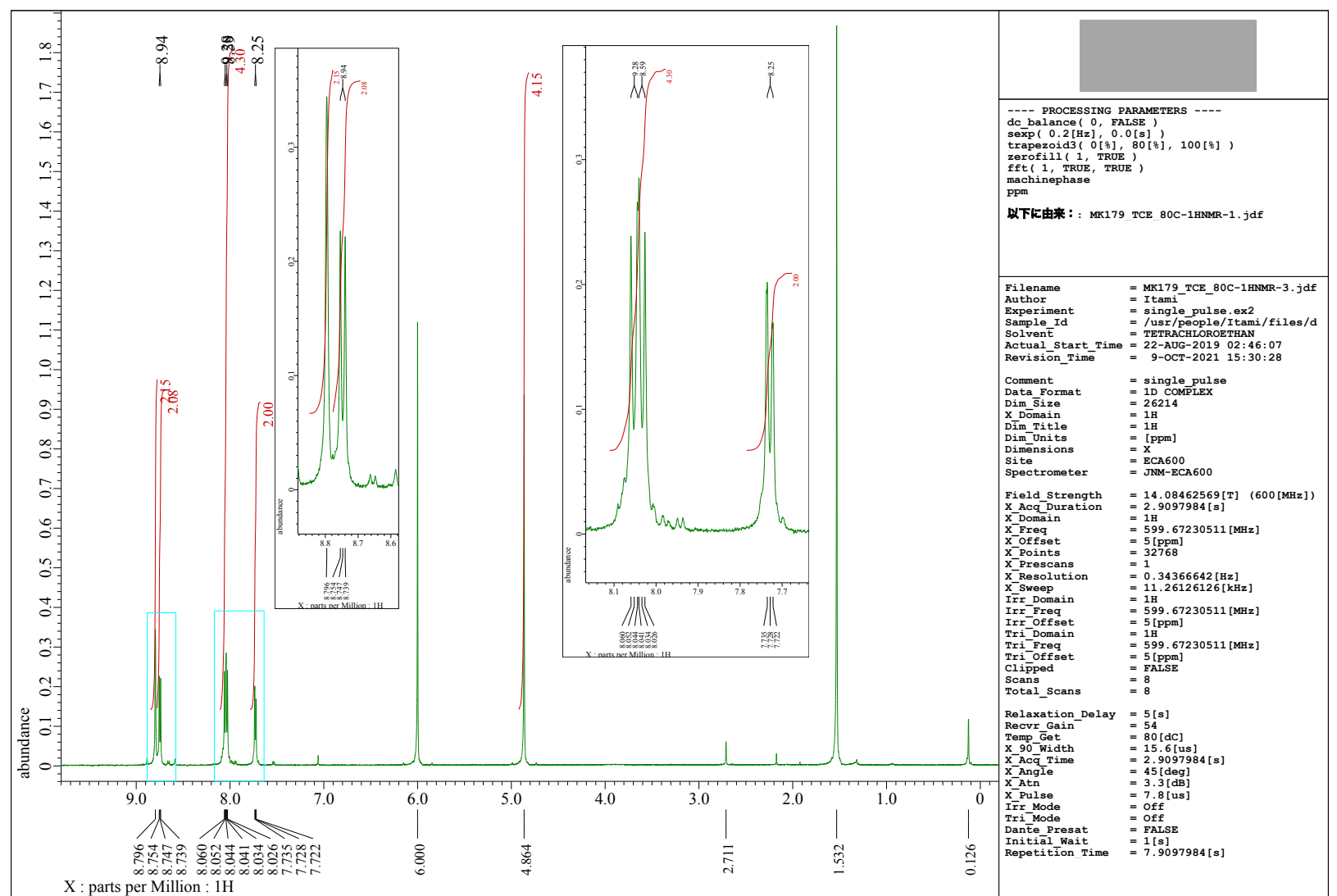

(b)

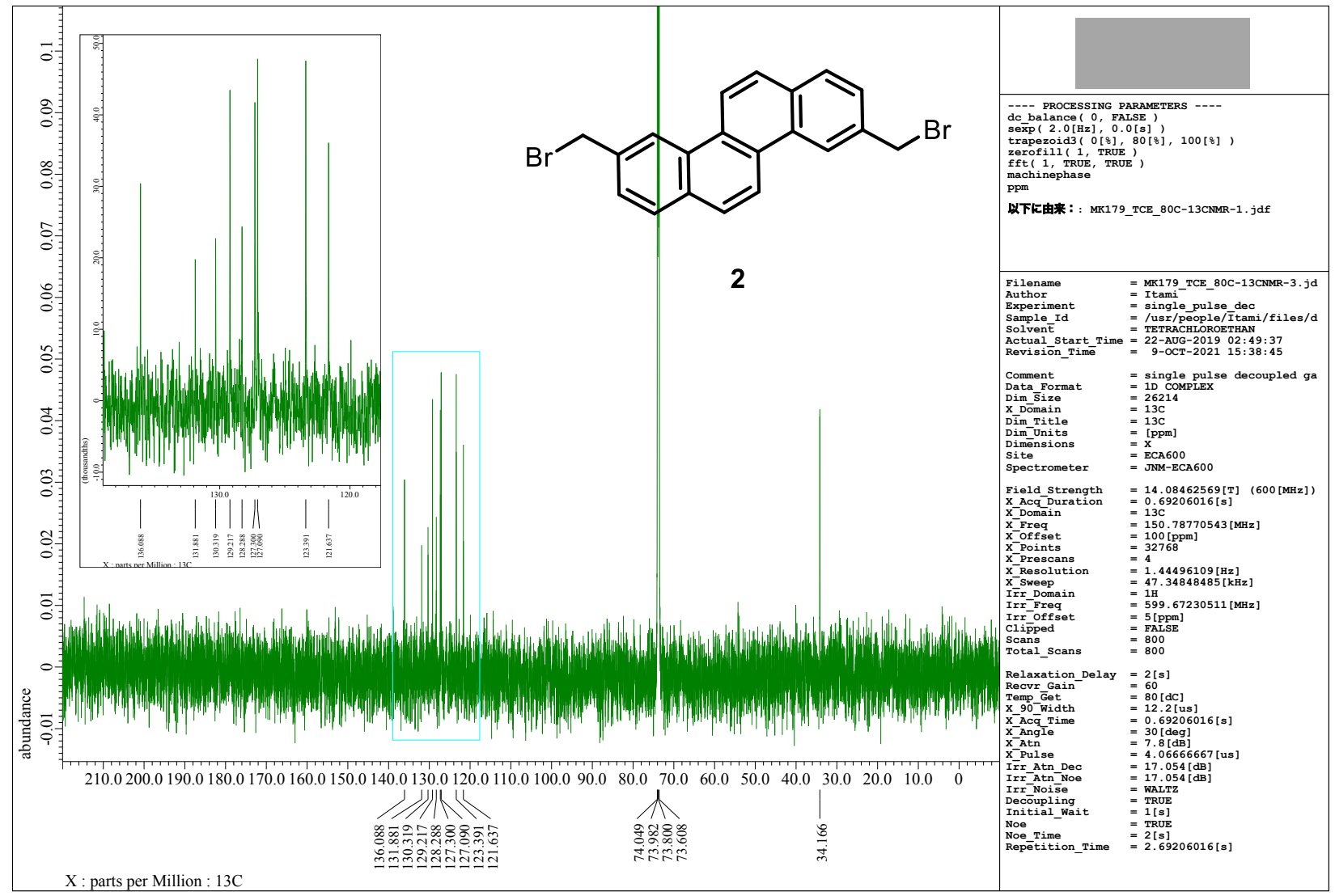

Figure S23. (a) ${ }^{1} \mathrm{H}$ NMR of $2\left(600 \mathrm{MHz}, \mathrm{Cl}_{2} \mathrm{CDCDCl}_{2}, 80{ }^{\circ} \mathrm{C}\right)$; (b) ${ }^{13} \mathrm{C} \mathrm{NMR}$ of $2\left(151 \mathrm{MHz}, \mathrm{Cl}_{2} \mathrm{CDCDCl}_{2}\right.$, $\left.80^{\circ} \mathrm{C}\right)$ 
(a)

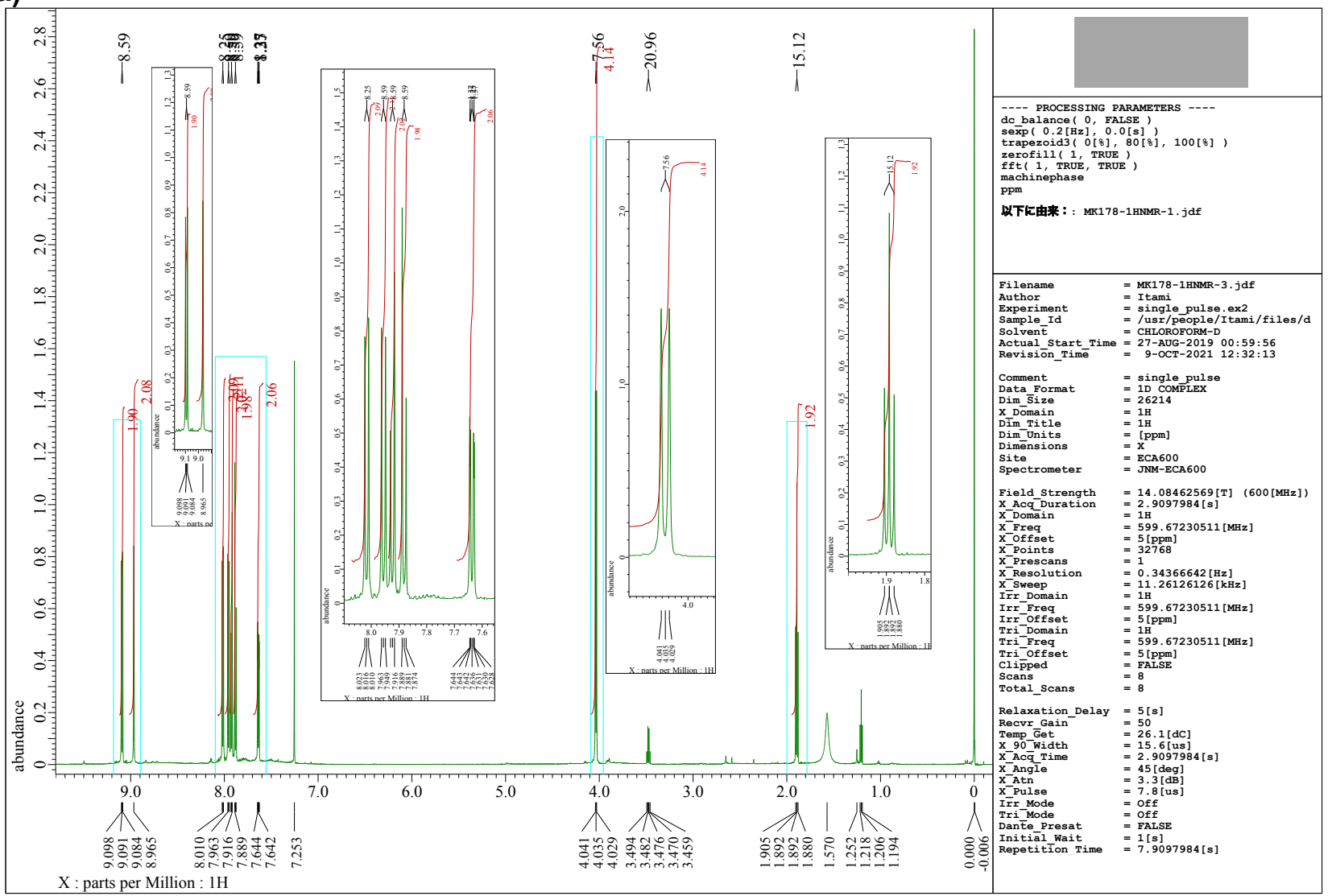

(b)

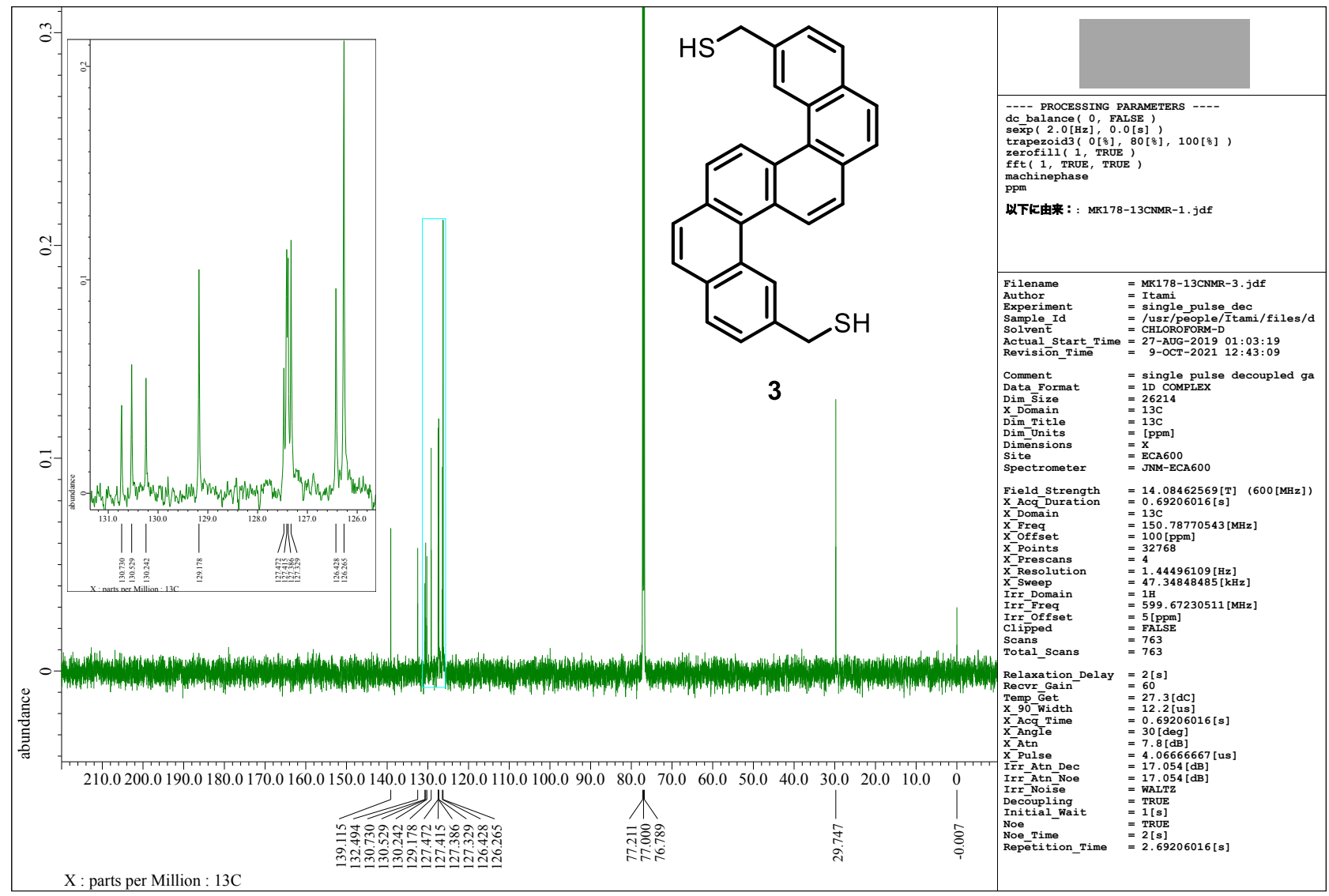

Figure S24. (a) ${ }^{1} \mathrm{H}$ NMR of $3\left(600 \mathrm{MHz}, \mathrm{CDCl}_{3}\right)$; (b) ${ }^{13} \mathrm{C} \mathrm{NMR}$ of $3\left(151 \mathrm{MHz}, \mathrm{CDCl}_{3}\right)$ 
(a)

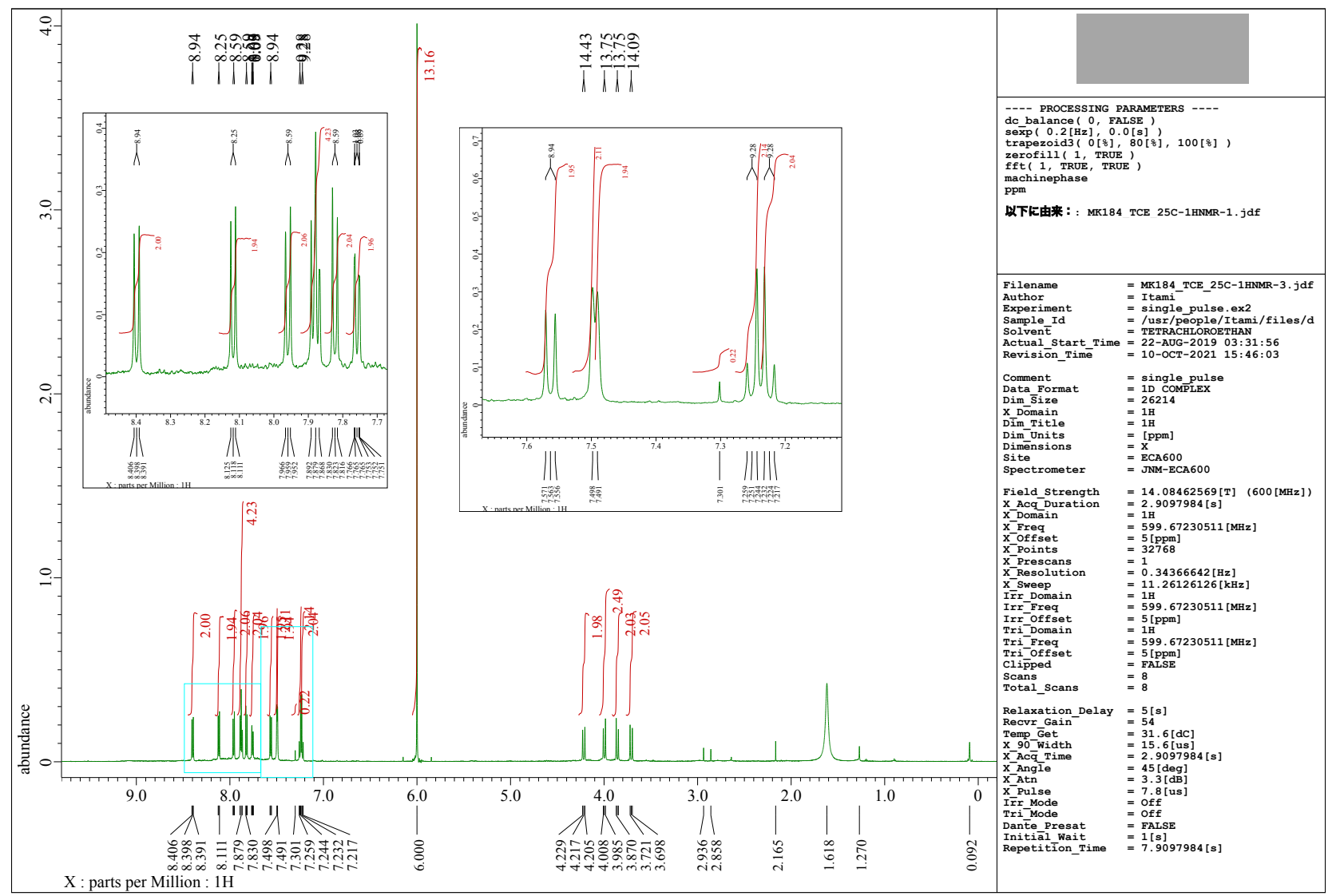

(b)

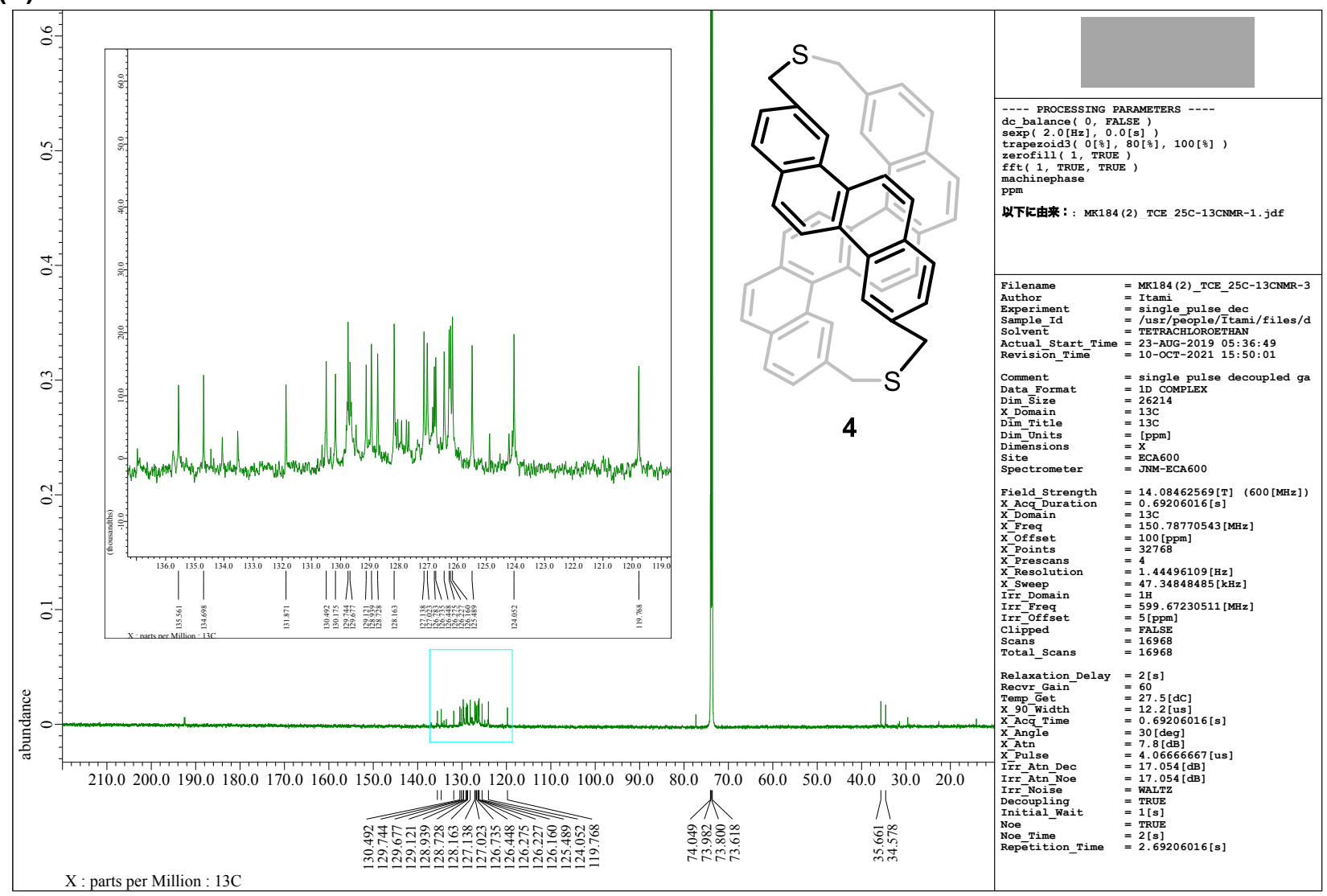

Figure S25. (a) ${ }^{1} \mathrm{H}$ NMR of $4\left(600 \mathrm{MHz}, \mathrm{Cl}_{2} \mathrm{CDCDCl}_{2}\right)$; (b) ${ }^{13} \mathrm{C} \mathrm{NMR}$ of $4\left(151 \mathrm{MHz}, \mathrm{Cl}_{2} \mathrm{CDCDCl}_{2}\right.$ ) 
(a)

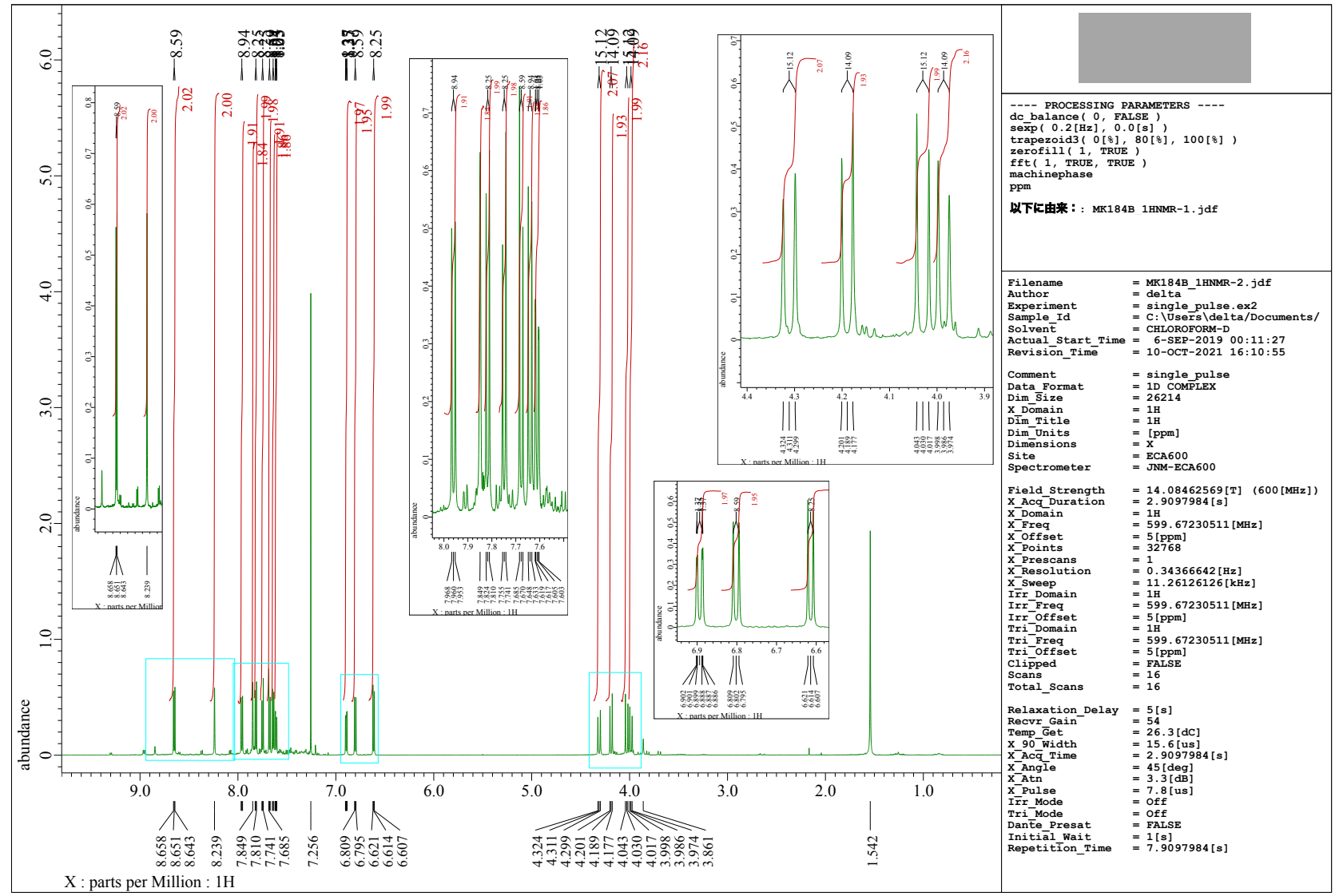

(b)

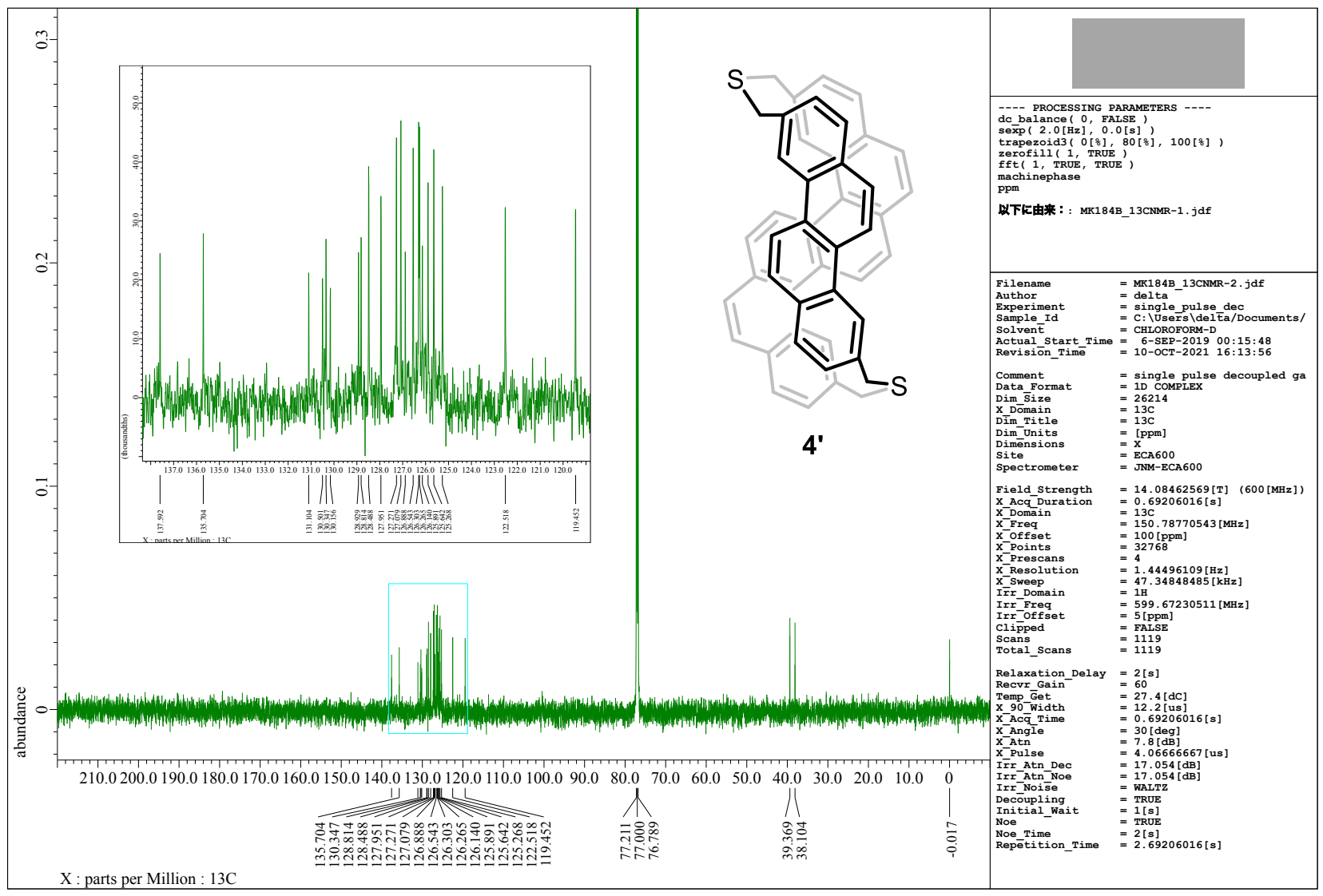

Figure S26. (a) ${ }^{1} \mathrm{H}$ NMR of 4' $\left(600 \mathrm{MHz}, \mathrm{CDCl}_{3}\right) ;(\mathrm{b}){ }^{13} \mathrm{C}$ NMR of 4' $\left(151 \mathrm{MHz}, \mathrm{CDCl}_{3}\right)$ 
(a)

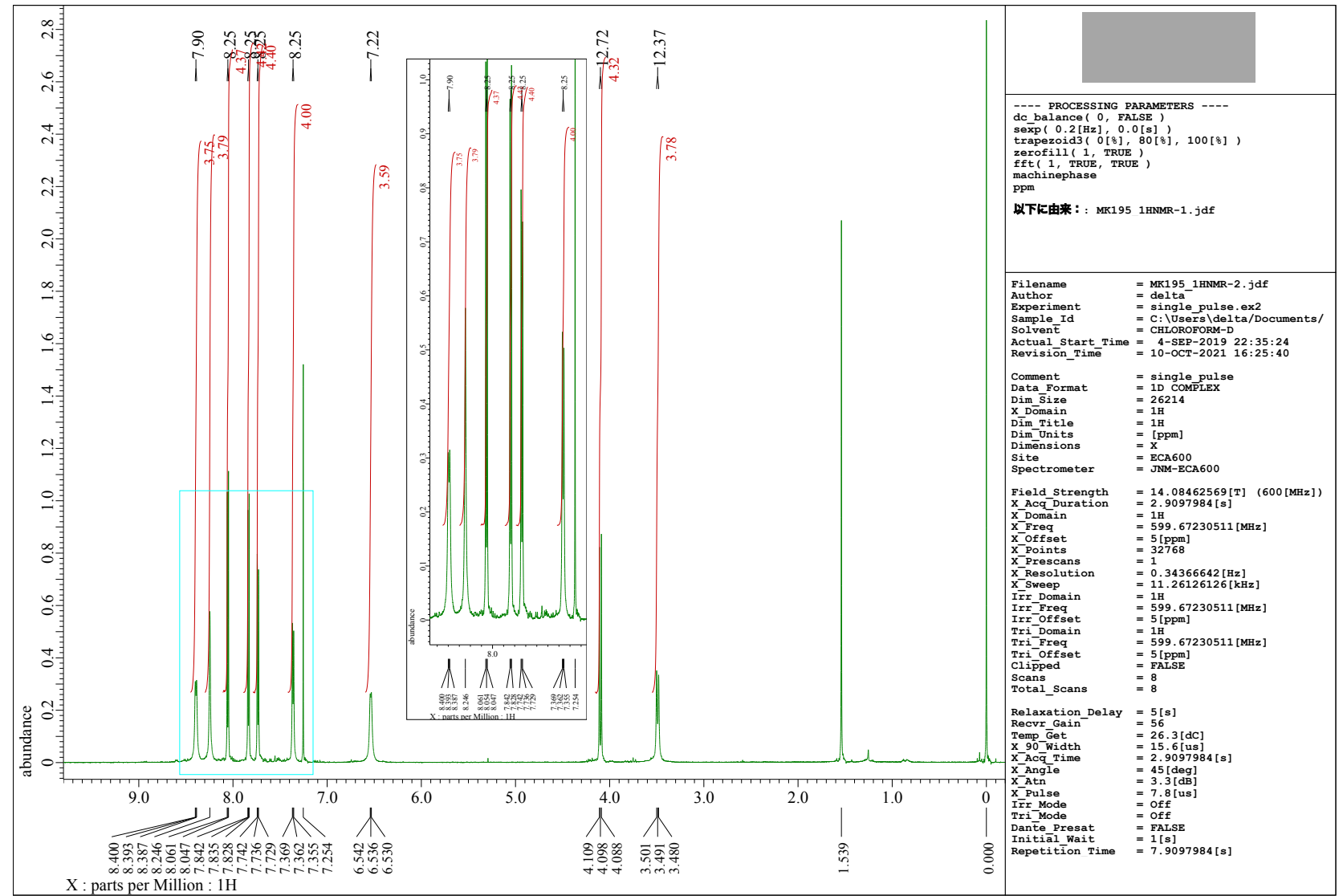

(b)

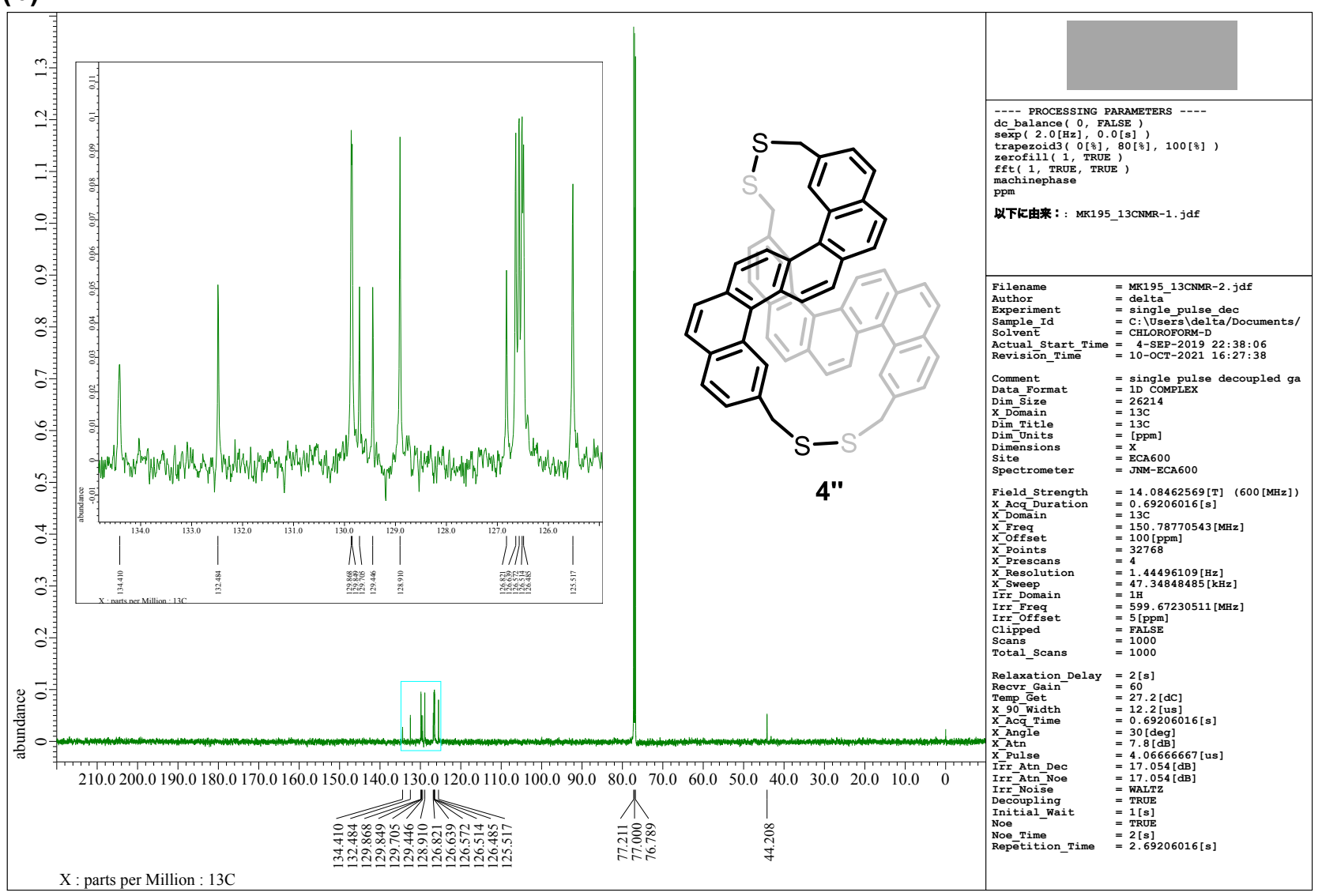

Figure S27. (a) ${ }^{1} \mathrm{H} \mathrm{NMR}$ of 4"(600 MHz, $\left.\mathrm{CDCl}_{3}\right)$; (b) ${ }^{13} \mathrm{C} \mathrm{NMR}$ of 4" $\left(151 \mathrm{MHz}, \mathrm{CDCl}_{3}\right)$ 
(a)

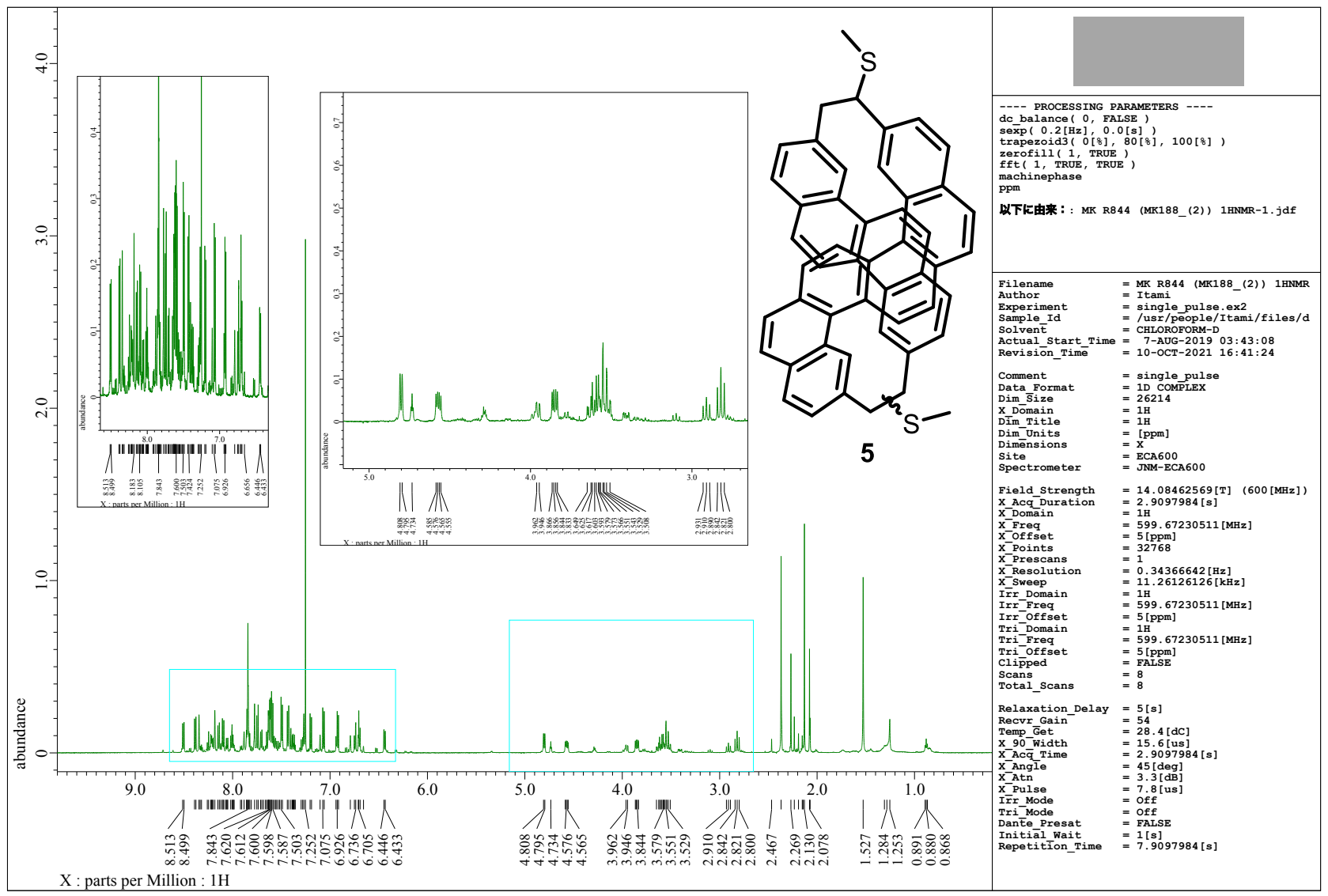

(b)

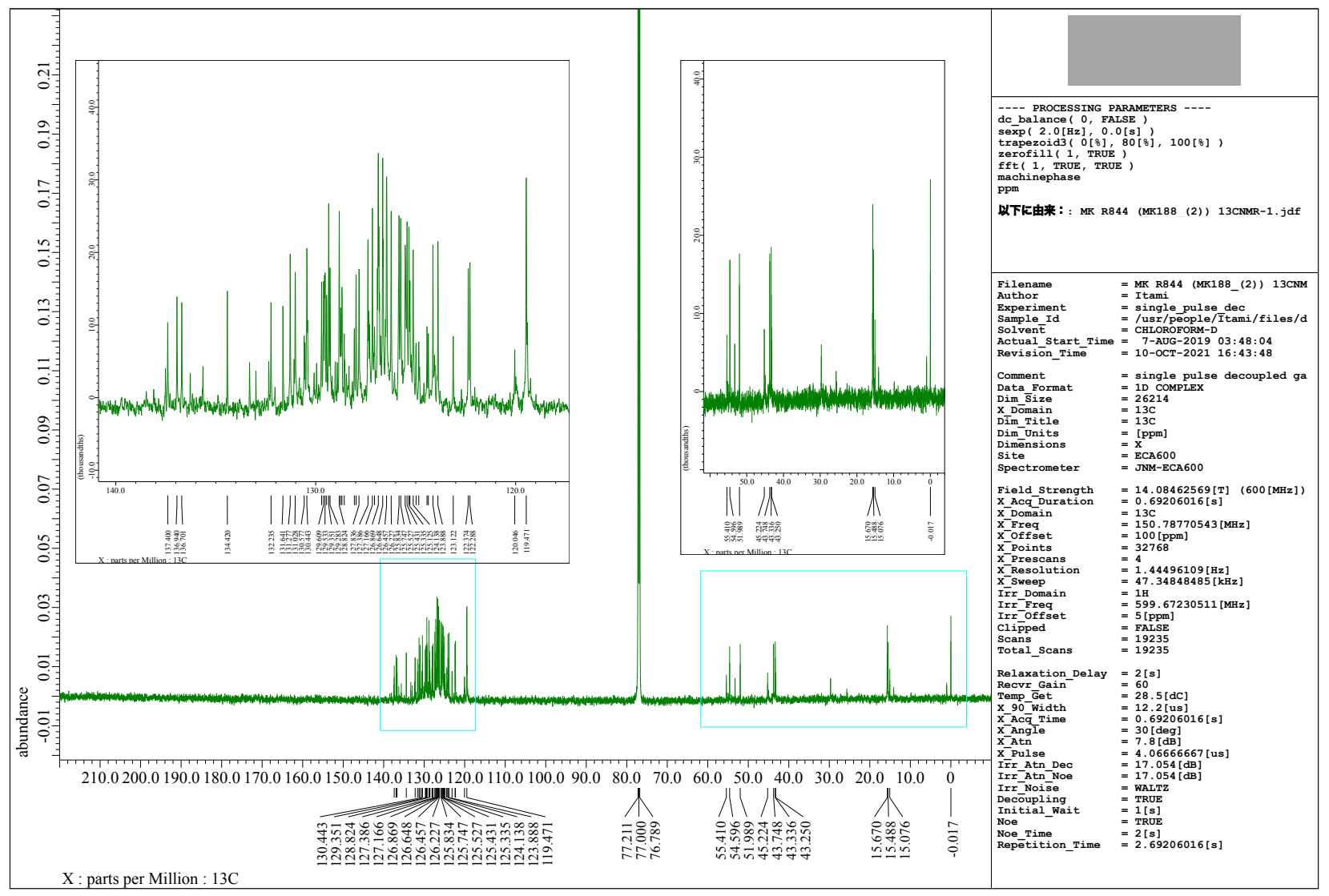

Figure S28. (a) ${ }^{1} \mathrm{H}$ NMR of $5\left(600 \mathrm{MHz}, \mathrm{CDCl}_{3}\right) ;(\mathrm{b}){ }^{13} \mathrm{C} \mathrm{NMR}$ of $5\left(151 \mathrm{MHz}, \mathrm{CDCl}_{3}\right)$ 
(a)

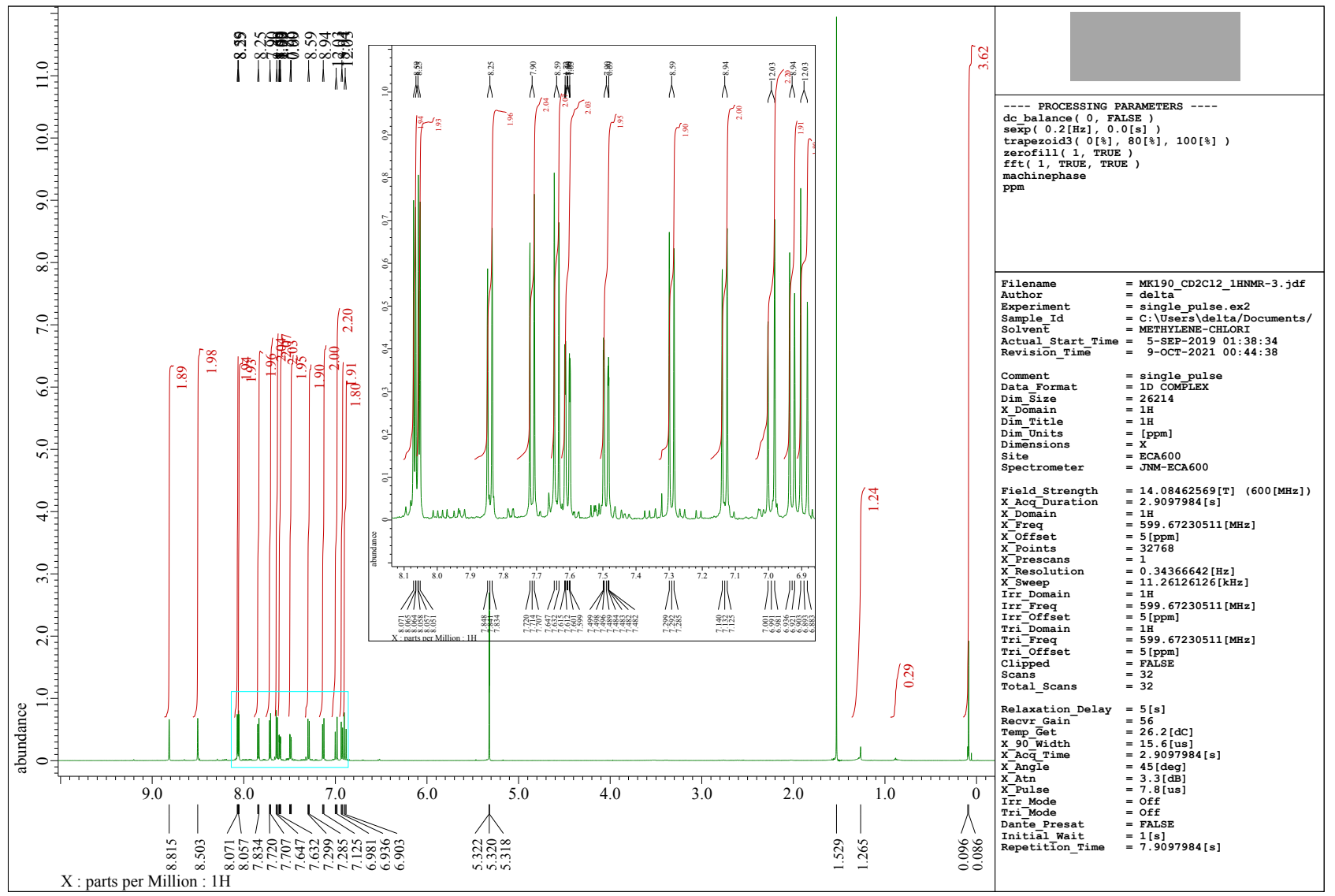

(b)

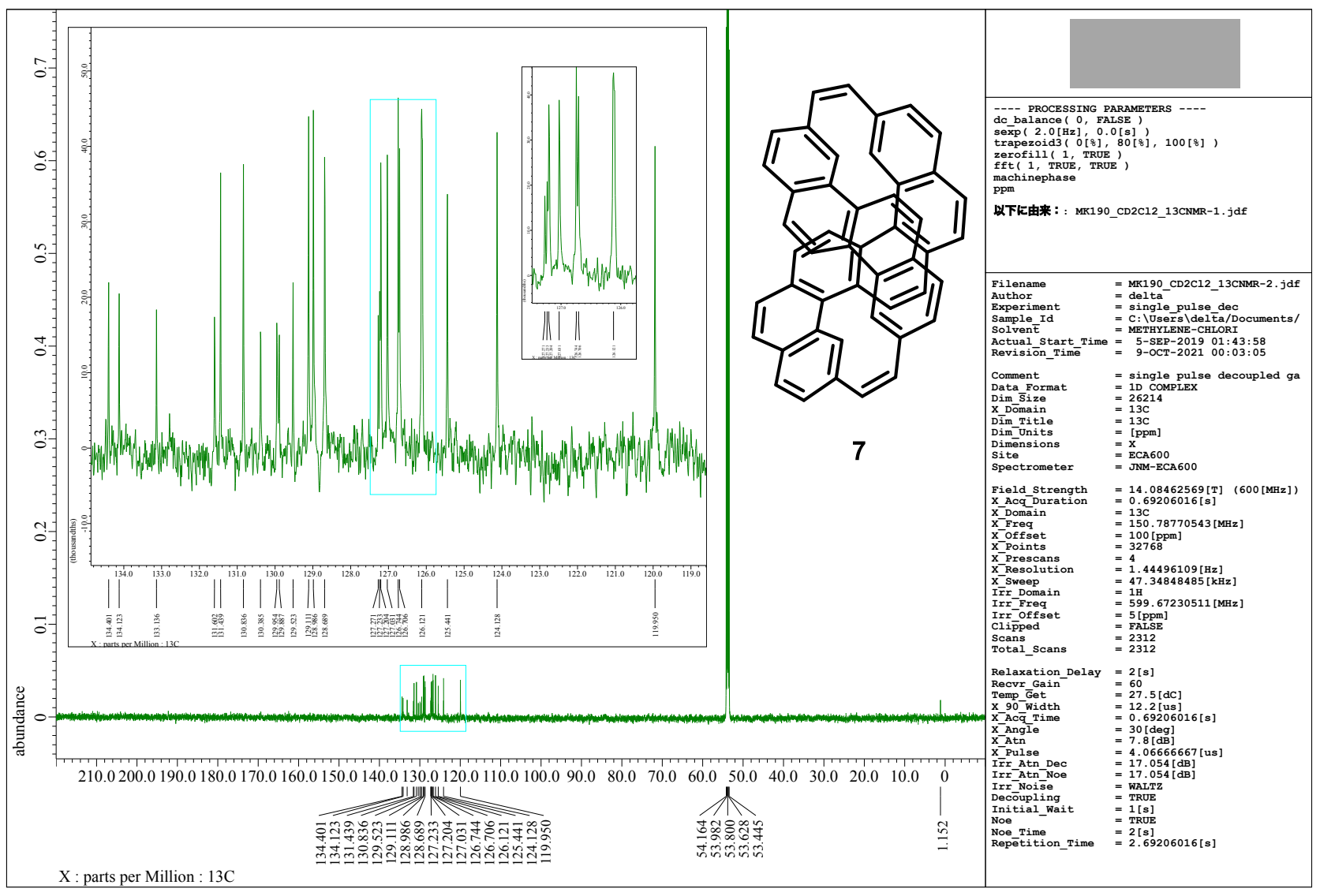

Figure S29. (a) ${ }^{1} \mathrm{H}$ NMR of $7\left(600 \mathrm{MHz}, \mathrm{CDCl}_{3}\right) ;(\mathrm{b}){ }^{13} \mathrm{C} \mathrm{NMR}$ of $7\left(151 \mathrm{MHz}, \mathrm{CDCl}_{3}\right)$ 
(a)

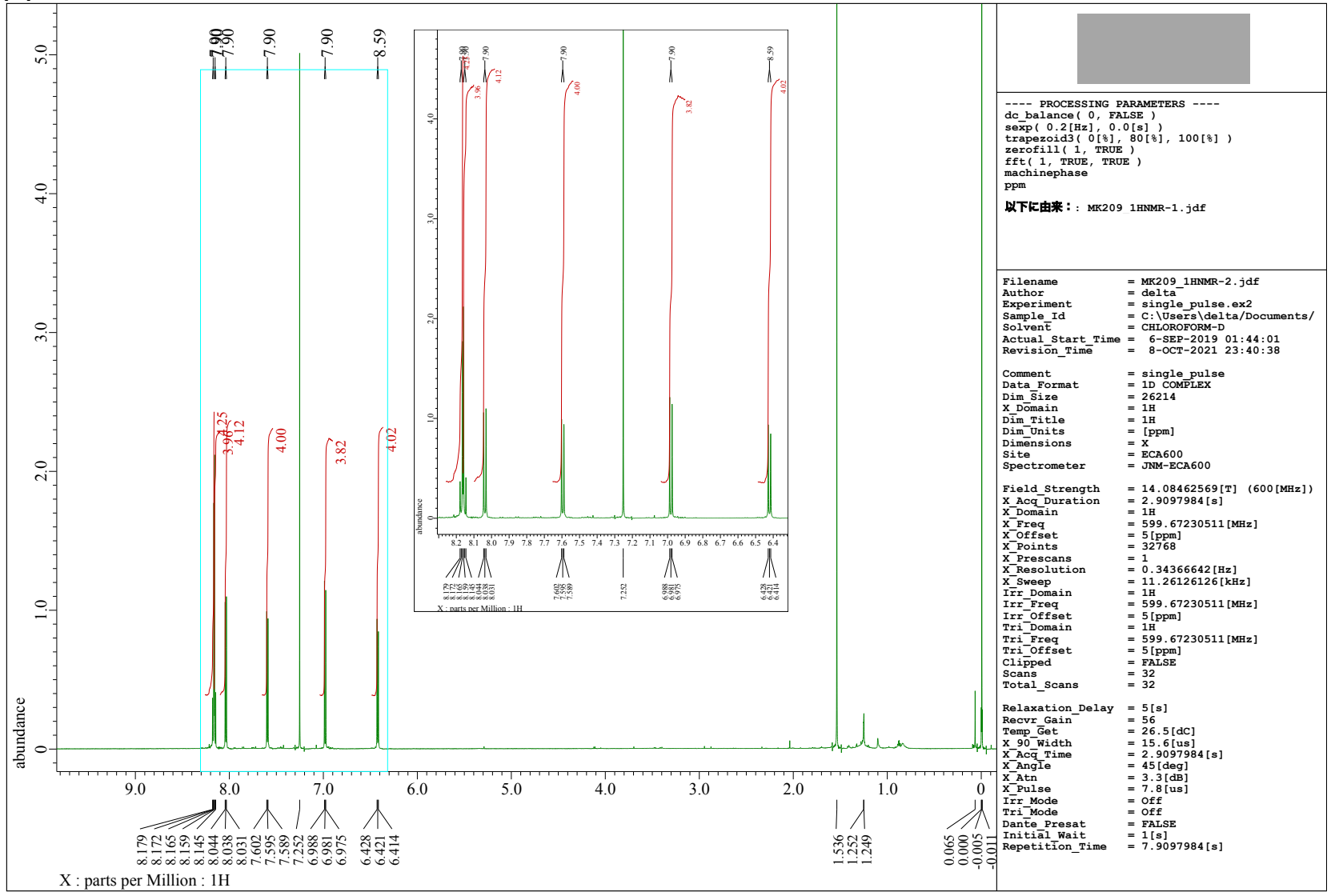

(b)

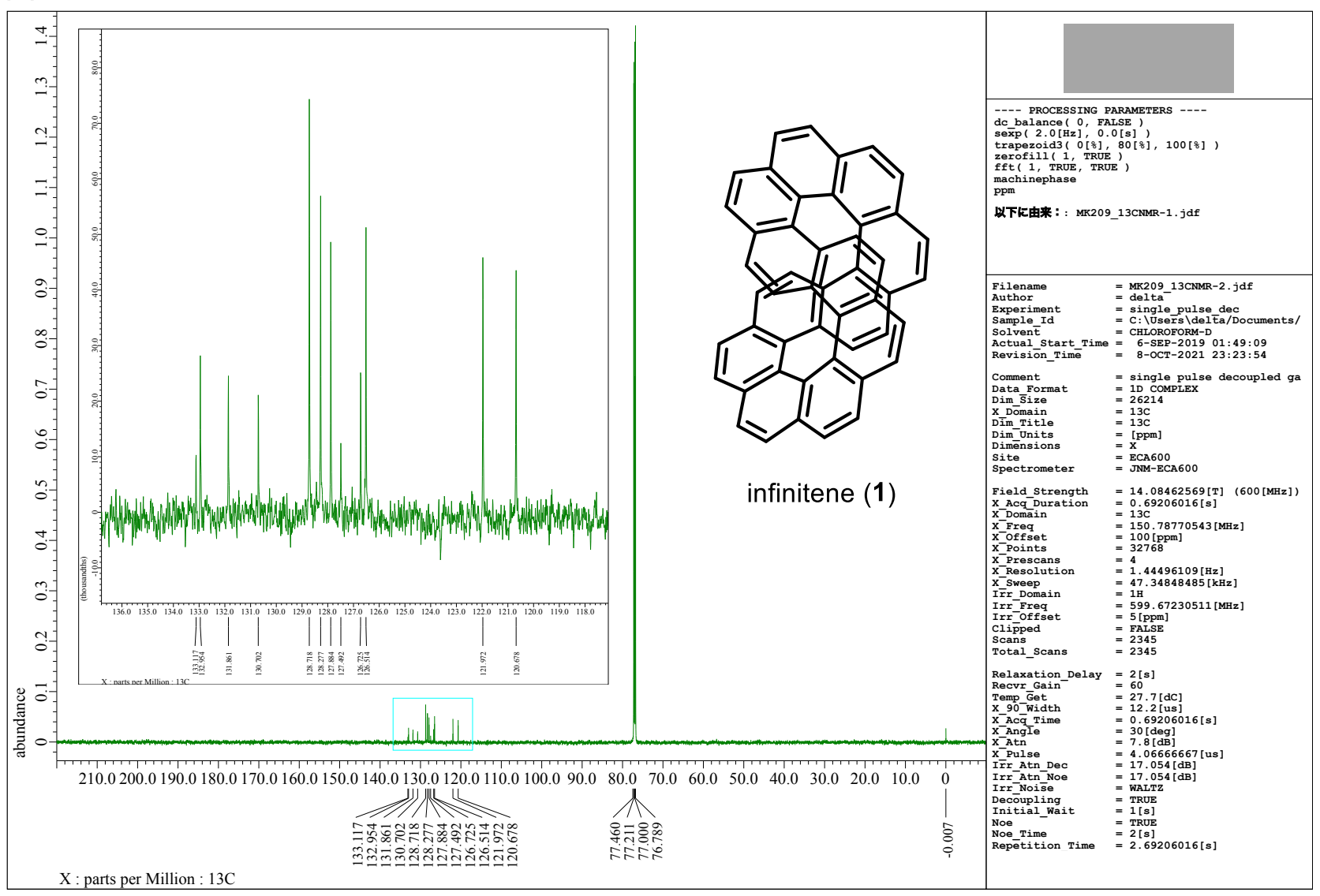

Figure S30. (a) ${ }^{1} \mathrm{H}$ NMR of $1\left(600 \mathrm{MHz}, \mathrm{CDCl}_{3}\right) ;\left(\right.$ b) ${ }^{13} \mathrm{C} \mathrm{NMR}$ of $1\left(151 \mathrm{MHz}, \mathrm{CDCl}_{3}\right)$ 